Florida International University

FIU Digital Commons

FIU Electronic Theses and Dissertations

University Graduate School

$9-1988$

\title{
A simulation of a message passing protocol for a network of transputers
}

Janice R. Glowacki

Florida International University

Follow this and additional works at: https://digitalcommons.fiu.edu/etd

Part of the Computer Sciences Commons

\section{Recommended Citation}

Glowacki, Janice R., "A simulation of a message passing protocol for a network of transputers" (1988). FIU Electronic Theses and Dissertations. 3830.

https://digitalcommons.fiu.edu/etd/3830

This work is brought to you for free and open access by the University Graduate School at FIU Digital Commons. It has been accepted for inclusion in FIU Electronic Theses and Dissertations by an authorized administrator of FIU Digital Commons. For more information, please contact dcc@fiu.edu. 


\section{ABSTRACT}

A SIMULATION OF A MESSAGE PASSING PROTOCOL

FOR A NETWORK OF TRANSPUTERS

by

Janice R. Glowacki

With decreasing cost and size of processors and more sophisticated demands of computer users, it is becoming popular to execute programs in parallel on a distributed network. Processors communicate through shared memory or hard-wired links depending on the hardware and topology of the system. Simulation is an appropriate tool for the investigation of system throughput, and the projection of system behavior under various workloads.

In this paper is described the configuration and communication protocol of an INMOS Transputer network, and the construction, verification, and validation of a detailed simulation model for the network. Results obtained from the execution of the model, projecting system behavior under both heavy and moderate workloads, are presented. The most significant results obtained indicate that system throughput is severely degraded when increases are made to either message traffic distance or network buffer size. Several areas for further research are suggested, including an alternative topology for large networks. 
A SIMULATION OE A MESSAGE PASSING PROTOCOL

FOR A NETWORK OF TRANSPUTERS

by

Janice R. Glowacki

A thesis submitted in partial fulfillment of the requirements for the degree of

MASTER OE SCIENCE

in

COMPUTER SCIENCE

at

FLORIDA INTERNATIONAL UNIVERSITY

Committee in charge:

Professor John Craig Comfort Chairperson

Professor David Barton

Professor Doron $\mathrm{Tal}$

September 1988 
To Professors John Comfort,

$$
\begin{aligned}
& \text { David Barton, } \\
& \text { Doron Tal, }
\end{aligned}
$$

This thesis, having been approved in respect to form and mechanical execution, is referred to you for judgment upon its substantial merit.

Dean James Mau

College of Arts and Sciences

The thesis of Janice R. Glowacki is approved.

Professor David Barton

Professor Doron Tal

Major Professor John Comfort

Date of Examination: September 16, 1988 
A SIMULATION OF A MESSAGE PASSING PROTOCOL

EOR A NETWORK OE TRANSPUTERS

\author{
by \\ Janice R. Glowacki
}

A Thesis submitted in Partial fulfillment of the requirements for the degree of

MASTER OF SCIENCE

in

COMPUTER SCIENCE

at

FLORIDA INTERNATIONAL UNIVERSITY

1988 


\section{ACKNOWLEDGEMENTS}

I wish to thank my husband, Paul, and Professor John Comfort, whose support and encouragement helped make this project a success. In addition, I give special thanks to Li Qiang and Raja Gopal for sharing their friendship and Transputer expertise. 
TABLE OF CONTENTS

LIST OF EIGURES . . . . . . . . . . . . . . . . vi

LIST OF TABLES . . . . . . . . . . . . . . . . .vii

1. INTRODUCTION .................. 1

DISTRIBUTED NETWORKS . . . . . . . . . . 1

SIMULATION . . . . . . . . . . . . . 2

SUMMARY OE PREVIOUS WORK ........... . 4

2. THE REAL NETWORK ............. . . 6

TRANSPUTER HARDWARE AND SOFTWARE . . . . . . 6

THE COMMUNICATION PROTOCOL . . . . . . . . 8

The Five Communication Processes

Avoiding Deadlock

Proof The Algorithm Is Deadlock-Eree 
3. THE SIMULATION MODEL . . . . . . . . . . 16 SIMULATION METHODOLOGY . . . . . . . . 16 SYSTEM REPRESENTATION . . . . . . . . . . 18 REEINEMENT OF THE SIMULATION MODEL . . . . . . 22 The Original Version The Second Version The Third Version The Final Version

4. VERIEICATION AND VALIDATION . . . . . . . . 26 MODEL VERIFICATION ............. 26 MODEL VALIDATION . . . . . . . . . . 28

5. RESULTS . . . . . . . . . . . . 32 SYSTEM PERFORMANCE UNDER DIFEERENT WORKLOADS * . 32 EFFECT OF BUEFER SIZES . . . . . . . . . 40

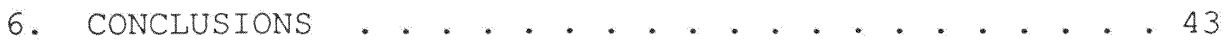


7. FURTHER RESEARCH . . . . . . . . . . . . . 45

APPENDICES

A. THE StATE DIAGRAMS . . . . . . . . . 48

The User Generator

The User Receiver

The User Front

The Network-In (Server)

The Network-Out (Transmitter)

B. THE NETWORK COMMUNICATION CODE . . . . . . . . 54

C. THE SIMULATION CODE............. . 70

LIST OF REFERENCES .................. . . . . . . 02

VITA . . . . . . . . . . . . . . . 103 


\section{IIST OF FIGURES}

Eigure

Page

1. Transputer network topology . . . . . . . . . 7

2. A single node in the network . . . . . . . . . 9

3. Pre-deadlock situation . . . . . . . . . . 14

4. Simulated network topology . . . . . . . . . . 17

5. Simulated Versus Real: Message Time in system . . 30

6. Workload Comparison . . . . . . . . . . . 34

7. Average Time in System: Moderate Load \#1 . . . 37

8. Average Time in System: Moderate Load \#2 . . . 38

9. Average Time in System: Heavy Load \#1 . . . . . 39

10. Average Time in System: Heavy Load \#2 . . . . . 40

11. Alternate topology for large networks . . . . . 45

12. Summary of State Diagram Symbols . . . . . . . . 48

13. The User Generator State Diagram . . . . . . . . . 49

14. The User Receiver State Diagram . . . . . . . . 50

15. The User Eront State Diagram . . . . . . . . . 51

16. The Network-In (Server) State Diagram . . . . . . 52

17. The Network-out (Transmitter) State Diagram . . 53 


\section{LIST OF TABLES}

Table

1. Simulated Versus Real: Average Message Time in System (Seconds) . . . . . . . . . . 29

2. Average Time a Message Remains in a Four-Node Network With Random Message Length (Seconds). . 31

3. Aggregated Average Time in System: All Loads (Miliseconds). . . . . . . . . . . . 36

4. Effect of Buffer Size for Worst Case Scenario (Miliseconds) . . . . . . . . . 41

5. Effect of Buffer Size for Best Case Scenario (Milliseconds) . . . . . . . . . 42 


\section{CHAPTER 1}

\section{INTRODUCTION}

\subsection{DISTRIBUTED NETWORKS}

Large computer networks, local area networks, and multiple processor systems are considered to be distributed networks. With these systems, processes of a single program can be distributed over several processors such that each processor on the network performs a subtask of the main program. Network processors need to share mutual information and are classified as tightly or loosely coupled [7]. Because tightly coupled systems have shared memory, an algorithm must exist to insure mutually exclusive access to it. Loosely coupled systems have local memory for each processor and communicate by using a message passing scheme.

Processors (nodes) in a ring network are loosely coupled and physically connected in a circle, usually with one-way communication links. Generally, a token or store-and-forward message passing scheme is used to support communication between nodes.

In a token passing scheme, a specific message, the token, continuousiy circulates through the network [7]. If a node wants to send a message, it must first acquire access to the network by removing the token when it arrives. This sending node forwards a message header followed by the 
message. When the message has traveled completely around the network, the sending node removes it (guaranteed the destination node received it) and forwards the token. Thus, only one message may travel through the system at one time.

With a store-and-forward message passing scheme, each node has designated storage (buffer) for incoming messages. As messages are received, they are placed in this buffer. When messages can be forwarded, they are removed from it. Because the buffer is a shared resource, the communication scheme is not trivial. The sending and receiving processes form a producer/consumer relationship and special techniques must be employed to prevent deadlock.

With advanced system architecture it is not uncommon to find systems with a large number of processors. The Ethernet ${ }^{1}$ local area network, for instance, can support up to 1024 processors [5].

\subsection{SIMULATION}

In order to analyze a network and evaluate system throughput or determine the number of processors needed for efficient communication, a simulation model can be designed. The behavior of a simulation system, according to Banks and Carson [1], "can be used to experiment with new designs or policies prior to implementation". Shannon [6] explains:

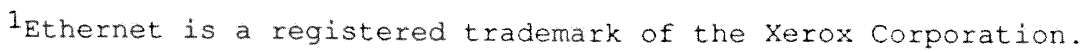


Simulation is the process of designing a model of a real system and conducting experiments with this model for the purpose either of understanding the behavior of the system or of evaluating various strategies (within the limits imposed by the criterion or set of criterial for the operation of the system.

Simulation models are classified as continuous or discrete-event. The difference is based on the way the state of the system changes over time. Continuous simulation is used to model a system that changes continuously over time. Discrete-event simulation is used to model a system which changes state at discrete intervals of time.

Banks and Carson explain a discrete-event simulation "proceeds by producing a sequence of system snapshots lor system images) which represent the evolution of the system through time" [1]. A snapshot for time (CLOCK $=t)$ includes:

* the system state at time t--the variables that describe the system and are needed for the study

* the Future Events Queue (FEQ)--the list containing all activities in progress and the time they will terminate

* the status of all entities--the objects of interest

* current accumulators and counters used for statistic summaries

In discrete-event simulation models, events are classified as bound or contingent. Bound events mark the ending of an activity of specified length. Contingent events are 
determined by certain conditions of the system and are triggered by the occurrence of a bound event.

\subsection{SUMMARY OF PREVIOUS WORK}

Several distributed systems have been simulated in order to evaluate their performance. The maximum mean data rates for several local area networks are presented by Stuck [8]. He explained that transmission medium has a dual purpose: to control access to the network and to transmit the data. Traffic on the network may be of low or high delay. When the network has high delay traffic, it is a bottleneck, and more time may be spent controlling access to the network than actually transmitting data.

Stuck included an evaluation of two ring networks and two bus networks. The ring networks consisted of 100 stations using a token passing scheme. The first had a single station sending to any of the 99 other stations, while the second had all 100 stations sending messages to each other. The bus networks consisted of a token passing scheme and carrier sense multiple access with collision detection. Stuck concluded by stating "Token passing via a ring is the least sensitive to workload, offers short delay under light load, and offers controlled delay under heavy load".

Garcia and Shaw [3] studied transient behavior of a five-node network using a store-and-forward message passing scheme. Assuming message traffic would be changing in the future, they were interested in analyzing current 
communication channels to determine if they were adequate for future loads. In addition they were concerned with how performance might be improved.

Both a sudden burst of messages and a sudden reduction in interarrival time for given periods were modeled. They found network performance severely degraded by these transient message loads. 


\section{CHAPTER 2 \\ THE REAL NETWORK}

The INMOS Corporation manufactures microprocessors specifically designed for parallel processing. These processors are called Transputers ${ }^{2}$ and can be put together as a distributed network connected by their fast, hard-wired communication links. Currently, the school of Computer Science at Florida International University has a four-processor distributed network of T414 Transputers.

\subsection{TRANSPUTER HARDWARE AND SOFTWARE}

According to the INMOS Transputer Reference Manual, these T414 Transputers context switch in a microsecond and perform approximately seven million integer/data move instructions per second [4]. The communication links between processors transmit data at a rate of 10 or $20 \mathrm{MHz}$ (individually switch selectable) with effective rates of .8 and 1.6 million bytes per second, respectively.

INMOS markets several different configurations of its Transputers. The University owns several INMOS B004 and INMOS B003 boards. The B004 board is an IBM PC/XT or PC/AT

2 Transputer is a registered trademark of the INMOS Group of 
add-in board containing one T414 Transputer with two megabytes of memory. In addition, it contains an IMS $\mathrm{CO} 2$ link adaptor which connects one of the T414 communication links with the Input/Output channel of the PC/XT or PC/AT. The PC can then be used as an Input/Output device and file server for the Transputer. For this reason, the T414 Transputer on the B004 board is referred to as the "host" Transputer.

The network of four T414 Transputers, each with 256 kilobytes of memory, resides on an INMOS B003 board. Each Transputer has four bidirectional communication Iinks which can be connected to other Transputers or local memory. Therefore, several topologies are available for a network of Transputers. The current topology of the network is shown in Figure 1.

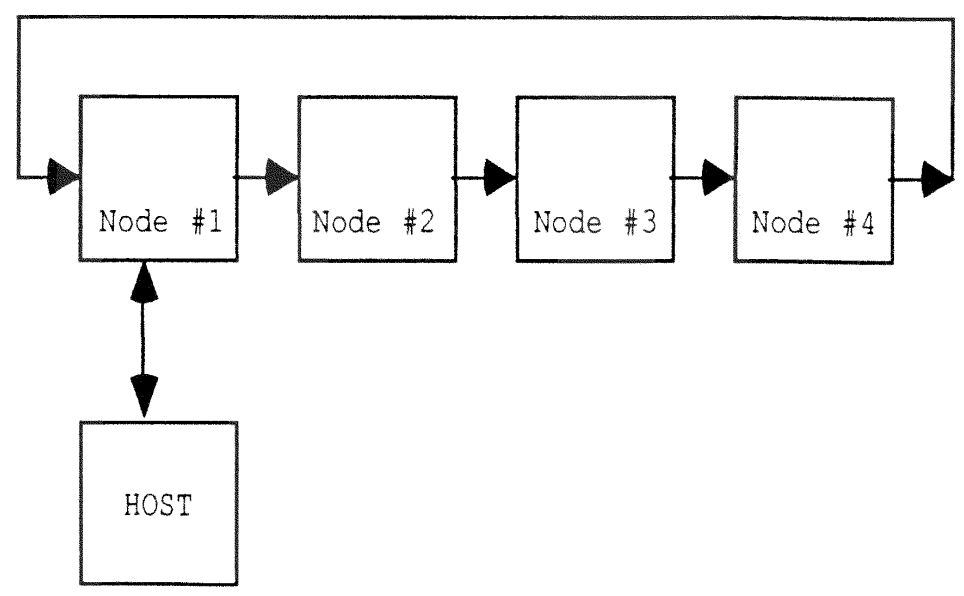

Eigure 1: Transputer network topology 
occam $^{3}$ is the native language of the Transputer system. The basic elements of an occam program are processes that can run sequentially or in parallel. Occam processes communicate over user-specified logical channels. These channels can be links connecting Transputers or local soft channels connecting processes running on the same Transputer. In addition, occam supports most of the constructs available in modern high-level languages.

One advantage of the occam view of processes is they are assigned to processors at compile time. Thus, a program developed as a set of parallel processes on a single Transputer system may be recompiled for any valid Transputer/process mapping [2].

\subsection{THE COMMUNICATION PROTOCOL}

A store-and-forward message passing scheme for the network of four Transputers was written by Li Qiang of Florida International University [9]. The system is comprised of five processes running on each node.

There exist two types of processes: network and local user. Network processes are those that have access to the physical links of the network. Local user processes do not have access to the physical network and are thereby "local" to a given node.

3 occam is a registered trademark of the INMOS Group of companies. 
There are three local user processes. The main one, performs the application program and generates messages for the node. The second receives all messages for the node. The third acts as an intermediate process supporting communication between the network and the receiving local process.

Figure 2 displays the five processes of a single node and shows the flow of message traffic through the network.

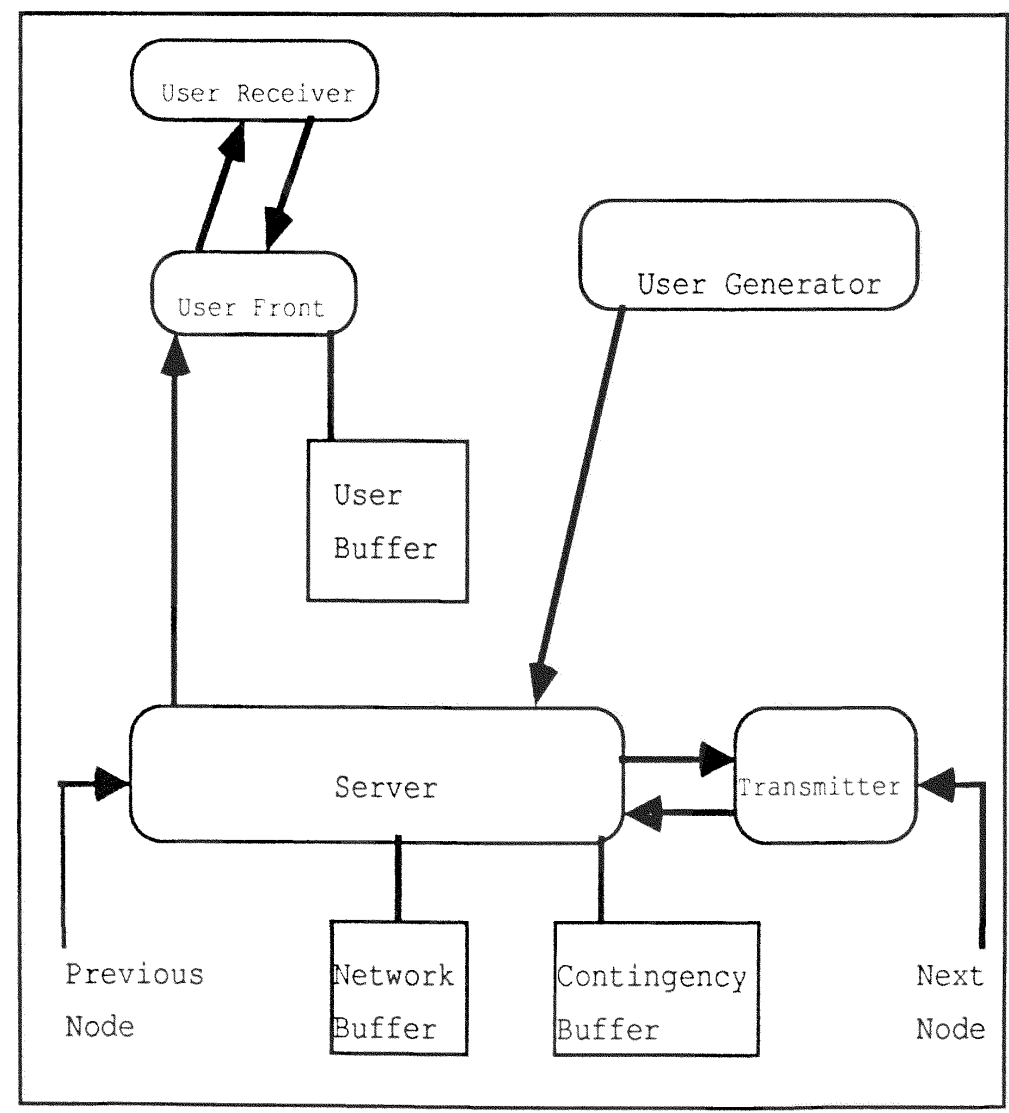

Figure 2: A single node in the network. 
In order to accommodate incoming messages, there exist three buffers: the user, the network, and the contingency. The user buffer contains those messages received for the local node. The network buffer holds those messages to be transmitted to the next node. The contingency buffer is a protective buffer holding a message that would otherwise overflow the network buffer. This buffer is necessary to avoid deadlock as explained by Qiang [9] and later in this chapter.

Each message contains a message header that indicates its source, destination, and length. The header itself is exactly one word regardless of the length of the message. It is important to note that messages are handled at the "word level". Each word of a message is sent individually although it is part of an entire message.

\subsubsection{The Five Communication Processes}

The primary responsibilities of the five processes shown in Figure 2 are explained below. To clearly identify each individual process, they have been named and underlined.

The User Generator. This process is responsible for creating messages and passing them over a soft channel to the server. The channel acts as a blocking channel. Therefore, the user generator is blocked between passing each word of a message.

The User Receiver. This process is responsible for 
reading the messages sent to the current node. It sends a request over a soft channel to the user front to read each word. It is therefore blocked from the time it sends a request until a word is actually forwarded.

The User Front. This process is responsible for the user buffer. It handles the producer/consumer relationship of the server and user receiver. The server passes words to the user buffer via the user front, while the user receiver gets words from the user buffer via the user front.

Occam channels are blocking channels. That is, if process $\mathrm{P} 1$ sends a word to process P2, P1 cannot continue until $\mathrm{P} 2$ receives the word. If $\mathrm{P} 2$ is busy and not ready to receive, then $\mathrm{P} 1$ remains blocked. In order to create a non-blocking channel, an intermediate process, P3, must be created [10].

Accordingly, in order to have the server (P1) pass messages to the local user receiver (P2) without blocking, there must exist the user front (P3) as an intermediate process. The user front takes messages from the server and, transparent to the server, places them in the user buffer. Upon request, it removes them from the buffer and forwards them to the user receiver. Because messages are handled at the word level, a separate request must be issued for each word of the message.

The server. This process takes words from the incoming link and places them in the appropriate buffer. Messages for the current node are sent to the user front and 
placed in the user buffer, while all other messages are placed in the network buffer for retransmission. It also receives messages from the user generator and places them in the network buffer for retransmission. Lastly, it answers the transmitter's requests by removing and forwarding messages from the network buffer (one word at a time).

The Transmitter. This process monitors the outgoing link. Whenever the link is available, it requests and receives a word from the server to be placed on the outgoing link.

\subsubsection{Avoiding Deadlock}

Deadlock can easily occur in this network if each user generator saturates the network to the point where every node is blocked from servicing incoming messages. In order to prevent this situation, there exists a protocol for filling the network buffer [9].

In short, the server receives messages from the user generator and the incoming link. Messages from the incoming link are categorized as "local" or "non-local". The server forwards local ones to the user front and fills the network buffer with non-local ones. The server places a message from the user generator into the network buffer if, and only if, the entire message can fit. Whenever the network buffer is full, however, the server blocks the user generator and processes messages from the incoming link by filling the contingency buffer. This buffer must be large 
enough to hold one complete message.

This protocol enables the server to push messages through the system even when the local user process has saturated the system. In other words, if the network buffer fills, the contingency buffer is still available to buffer network traffic.

The Transputer link, like a soft channel, behaves as a blocking link. Therefore, any word sent down a link remains on it until removed by the next node. For deadlock to occur, each link must be transmitting data, and each buffer (network and contingency) must be full such that every node is blocked and will remain blocked indefinitely. To avoid this situation, it is necessary to have the priority scheme for filling the network and contingency buffers as described.

\subsubsection{Proof The Algorithm Is Deadlock-Free}

The store-and-forward message passing algorithm by Qiang is deadlock-free [9].

Proof by contradiction. Assume the algorithm is not deadlock-free and the network is in the state of deadlock. In other words, each network and contingency buffer is full, each link has data on it, and each user generator is blocked from submitting a message into the network. Then, there is a situation just before deadlock similar to that shown in Figure 3. 


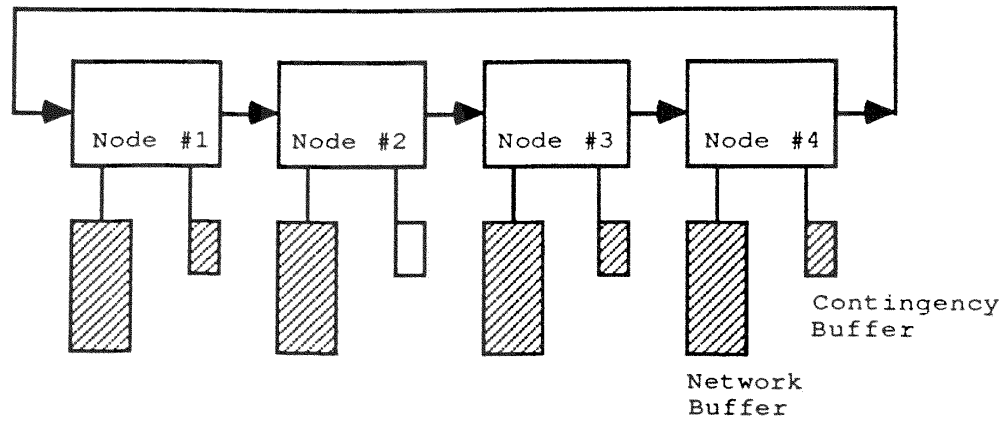

Figure 3: Pre-deadlock situation.

Suppose the last node to fill its contingency buffer was node \#2. Then, when node \#2 removed data from the incoming link it would enable node \#1 to move data from its network buffer to its outgoing link, transfer data from its contingency buffer to its network buffer, and receive the data on its incoming link to be placed in its contingency buffer. But then the network is not in a state of deadlock.

Contradiction of assumption. Hence, the algorithm is deadlock free.

When the network buffer is full, the algorithm's protocol requires the data from the incoming link be received before submitting to the network messages generated by the local node. This way, it guarantees flow of traffic even when the network is saturated with messages.

When the pre-deadlock situation occurs, filling node \#n's contingency buffer enables node \#n-1 to unload data from its network buffer and transfer contents from its contingency buffer. Thus, node \#n-1 now has an empty 
contingency buffer to place data from the incoming link. This will continuously propagate such that there is never an instance where each contingency buffer is full. Thus, when traffic is intense, the network can become blocked. However, because of this protocol for filling the network and contingency buffers, the network cannot deadlock. 


\section{CHAPTER 3}

\section{THE SIMULATION MODEL}

Simulating a network communication protocol requires complete understanding of both the real system and of simulation techniques. The simulation is not a duplication of the system with added statistical computations. Instead, it models the real system by recording and gathering statistical information based on the events and actions that would be occurring in the system. The computer programs for both the real and simulated systems are given in the Appendices in order to exemplify the significant difference between them.

\subsection{SIMULATION METHODOLOGY}

It is not uncommon for a simulation to use an enormous amount of computing time due to the number of calculations used for generating random numbers, accumulating statistics, and managing the future events queue. One attractive solution to shortening the run-time of a simulation is to incorporate a network of computing power. Comfort has investigated the idea of distributed simulation whereby related processes of the simulation can be placed on separate processors of a network [2].

Comfort has written a distributed simulation package 
to run on the INMOS Transputer system [2]. The program identifies objects such as a statistics module, random number generator, and a priority queue handler. Each object is a unique process. The program can be run on a single Transputer system; however, when running the simulation on a network of Transputers, it is possible to distribute each object onto separate processors of the network and enjoy the benefit of decreased run-time.

A simulation program using this package must first instantiate specific instances of these objects. The future events queue is an instance of a priority queue. The objects are then accessed by standard calls. Statistics are updated for an entity in the simulation by sending messages to the statistics package whenever the entity changes its state.

A comprehensive simulation model, using Comfort's package, was designed to investigate system throughput of the four-node ring network on the INMOS B003 board. The topology is shown in Figure 4 .

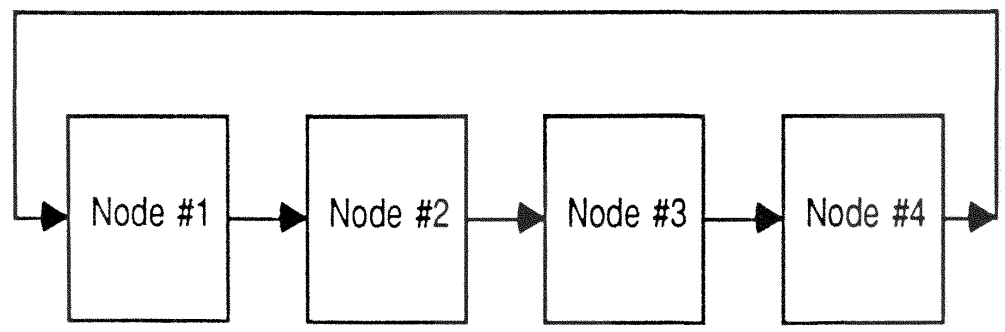

Figure 4: Simulated network topology. 
Qiang's message passing protocol, as described in the previous chapter, is modeled. Also of interest were the effects of message length variation, message traffic destination (distance a message travels), and system workloads.

\subsection{SYSTEM REPRESENTATION}

This section explains how the processes, links, buffers, and messages were represented in the simulation model. In addition, timing of the network and parameters of the simulation are discussed.

The servers and entities. In order to simulate the real network it was necessary to determine how processes and messages should be represented. As processes service messages in the real network, servers process entities in the simulation model. Each server required a set of states and well-defined actions to be performed.

Although processes on the same processor are conceptualized as running in parallel, only one process can actually be running at a time. Thus, for every node in the model, only one server (process) could be servicing (running) at a time. Each type of server had a designated set of states and actions describing the process being modeled and could therefore be in only one state and perform only one action at a time.

Messages in the system. Messages in the real network consisted of two parts: the message header and message 
body. The header contained the source, destination, and length of the message. In the simulation model, each message header was an entity.

Simulating the buffers. Physically, the network and contingency buffers comprise one buffer and are logically separated in software. Because the contingency part was required to accommodate the largest message size, the total buffer space needed had to be at least as large as two maximum size messages (one for each part of the buffer). Let the term network buffer now refer to the combination of the contingency and network buffer.

In order to model the user and network buffers that held messages, it was necessary to create one FIFO queue for each buffer of every node. These queues held the message header entities while local counters were updated to track the total words in a given buffer.

Simulating the links. A Transputer link could only hold one word at a time (message headers were single words). Because actions performed depended on the type of data sent, links were simulated using two variables. The first variable indicated the type of data on the link: a message header, a word of the message body, or indication the link was free. If a message header was on the link, then it was necessary to identify the actual entity number. This was held in the second variable.

The Future Events Queue. A single future events queue ( $E E Q$ ) held the bound event notices for the entire 
simulation. These notices included scheduling processes to time-out while waiting for a channel or because their run-time expired. Also included were notices from a node to another indicating data was sent down or removed from the link. In addition, there were batch run termination notices, as well as several others.

system timing. The time needed to perform each action was not easy to determine. Each Transputer cycle took about 67 nanoseconds which evaluates to 15 million cycles per second. In order to acquire accurate results, it was necessary to determine the time needed for each server to perform its various actions. The level of detail was so crucial that code for each process in the real network communication program was thoroughly evaluated to the point where instructions were literally counted [9]. In addition, the INMOS Reference manual was consulted for system timing statistics [4].

System clock. The simulation clock time referenced Transputer cycles rather than seconds. This was because each activity was evaluated in terms of the number of cycles necessary. If activity times were measured in nanoseconds, the clock time would become too large for some simulation runs. If activity times were measured in microseconds, then each activity would be rounded individually. Because each activity is performed a significant number of times each second, over or under estimating a time value would become significant. In order to minimize losing integrity in the 
times estimated, it was decided to keep all times in reference to Transputer cycles. As a result, a single simulation clock tick evaluated to 5 Transputer cycles. Thus, to simulate one second of real time, the simulation would have to run for time $=3,000,000$.

Random number generators. There were five random number streams used for the model. Each stream required the mean, seed, and distribution type. There were three possible distributions: constant, negative exponential, or uniform. The streams were used to generate numbers for:

* Average links a message travels (distance)

* Number of messages to send at once

* Length of the current message

* Time to run the local user application

* Operating system delay to schedule a process

Parameters to the system. The system required 23 parameters. They were:

* The number of nodes in the network (2 to 32)

* The speed of the links ( 10 or $20 \mathrm{MHz}$ )

* The number of batches to run

* The length of each batch

* The maximum length of a message

* The number of messages to send at once

* The size of the network buffer

* The size of the user buffer

* The distributions, means, and seeds, for each of the five random number streams 


\subsection{REFINEMENT OF THE SIMULATION MODEL}

To simulate a computer system it is necessary to decide the level of detail which will be modeled. Specifically, "the circuit level, gate level, register-transfer level, and system level" [3]. The initial simulation model was revised several times. Each revision increased the level of detail modeled. The state diagrams and a description of the bound event actions for the final version are given in the Appendix.

\subsubsection{The original Version}

In the original model there were three servers. One for each network process and one to represent all local user processes. The model itself would deadlock even though the real network did not.

The reason the simulation would deadlock is relatively simple and can be seen in the following scenario. Suppose each link contained a word being sent to the next node, and each contingency buffer was full. Furthermore, suppose node \#n was the last node to fill it's contingency buffer. Then, the last bound event was for the server of node \#n to place a word from the incoming link into the contingency buffer. The key here is the link between node \#n-l and node \#n. Because the last bound event was for node \#n, node \#n-1 was not aware of the change in status of its outgoing link. It is possible for all servers on node \#n-1 to be blocked. In such a case there would be no bound events for that node on the FEQ. Contingent events for node \#n are only checked 
after a bound event has been processed for node \#n. Therefore, if no bound events are scheduled for a node, then it can never reevaluate the status of its outgoing link. Hence the simulation could deadlock.

\subsubsection{The second Version}

The second version eliminated the possibility of deadlock in the simulation. The "fix" was quite simple although not elegant. After a bound event was processed for node \#n, the conditions for contingent events were checked for both node \#n and node \#n-1. Thus, the sending node would be able to update the status of the link when the receiving node made the link available. As expected, run-time of the simulation program was effected.

This model did not reflect the real network statistics as the simulated results were off by at least a factor of 5. All local user processes were handled as one server in the simulation and could not accurately reflect the real network. This was because the simulation did not account for the time needed for a context switch. In other words, the simulation modeled three separate processes running each for time $t$ as one process running for time $3 t$. In reality, it requires time $3 t+2 c$ where $c$ is the time for a context switch to occur between running processes. clearly, $3 t+2 c$ is strictly greater than $3 t$.

\subsubsection{The Third Version}

In the third model, two servers were added, separating 
the three local user processes and clearly defining the duties of the user receiver, user generator, and user front. This version attempted to adjust the timing problem in the previous version. Although the simulation results were significantly closer to the real network statistics, it was clearly evident another level of detail needed to be modeled.

\subsubsection{The Final Version}

Unless a priority scheme for scheduling servers was represented, an unrealistic ordering occurred in the simulation. Therefore, it was necessary not only to keep track of the servers that could process an entity (message), but also the order in which they became available to do so.

For this reason, two queues were added in the final model: Block and Ready. The Block queue held those servers waiting for some event or condition to occur before they could run, while the Ready queue held those servers which could be run. The servers in the simulation were placed on the block queue after serving an entity (message) and moved to the ready queue according to pre-defined conditions for the process being modeled. Essentially, this modeled the operating system's scheduler.

After a bound event was processed, the status of each server on the Block queue (for that specific node) had to be evaluated in order to determine which servers, if any, needed to be moved to the Ready queue. Then, if no servers 
were currently running, one from the Ready queue was scheduled.

Although this approach modeled the network more realistically, it did add several drawbacks. First, significantly more computations were being performed and as a result, program run-time was severely degraded. Second, as contingent events were not tested in the "traditional" scheme, the simulation would deadlock in the same manner as the original model. Therefore, it was again necessary to design a technique to avoid deadlock in the simulation.

There were two solutions investigated. The first one would require moving node \#n-1's transmitter from the Block queue to the Ready queue whenever node \#n removed a word from the link. However, there did not seem reasonable justification to manipulate a node's data structures while processing events of another node.

The second solution required an additional bound event notice to be scheduled. Although sending node \#n could compute the time a word would arrive at node \#n+1, it could not determine when the word would actually be removed. Therefore, whenever node $\# n+1$ removed data from the link, it was required to create and schedule a bound event notice for node \#n indicating the link became available. 


\section{CHAPTER 4 \\ VERIEICATION AND VALIDATION}

The simulation model must be verified and validated. Model verification deals with verifying the code performs accurately and is implemented correctly. Model validation deals with showing the code accurately models the real system. The previous chapter discussed the several versions of the simulation model. Each version was carefully evaluated in an attempt to verify and validate it. However, the earlier versions did not accurately model the real network and the revisions became evident during the evaluation process. This chapter discusses the verification and validation of the final version.

\subsection{MODEL VERIFICATION}

Verifying the simulation model, like verifying any computer program, can be done using very common sense techniques [1]. Banks and Carson suggest:

* make the code "self-documenting"

* make a flow diagram indicating the possibilities encountered when an action for an event occurs

* verify the input parameters are not modified

* use a program trace while testing the code

* closely examine the output for "reasonableness" 
Each of these techniques were incorporated in order to verify the simulation code. An explanation of the use of each techniques as it was applied to this project is given here.

Self-documenting code. An Occam program is viewed as a single fold comprised of other folds. A fold is simply the concept of grouping information or code together as a separate unit. Each fold can be identified with a name (generally used to explain the fold's contents) and may contain other folds, comments, and code. In general, folds are kept small and concise. Therefore, Occam programs are "self-documenting" by nature.

The code for the simulation program is given in the Appendix. Along with explanatory fold names, documentation for all variables, states, and actions were included in the source code.

Elow diagram. A flow diagram is suggested in order to evaluate each possible action the system can perform after each event. The flow diagram for the simulation model consists of the state diagrams for each of the servers. These can be found in the Appendix.

Verify input parameters. The 23 input parameters for the system were printed after several tests to verify they were not modified during the execution of the simulation.

Trace the execution. The trace was used to get output while the simulation was running to determine if the code was performing accurately. The trace was very useful and 
helped determine the reason the simulation would deadlock. In addition, it helped identify the unfair scheduling of processes in the earlier versions.

The trace included information about each queue (what was being added or removed from it), each random number stream (what stream was generating numbers and what the numbers were), the statistics package (what entity was entering and leaving what state), and each bound event action (what and when it was pulled from the $F E Q$ ).

Examine the output. The output for each version was evaluated. It was not until the final version that "reasonable" results were found. These results are explained and shown in the validation part of this chapter.

\subsection{MODEL VALIDATION}

Validation is an approach used to determine if the model accurately represents the real system. According to Banks and Carson [1]:

Validation is usually achieved through the calibration of the model, an iterative process of comparing the model to actual system behavior and using the discrepancies between the two, and the insights gained, to improve the model. This process is repeated until model accuracy is judged to be acceptable.

The rest of this chapter presents the results obtained from both the real and simulated networks. The results are compared and the simulation is "judged to be acceptable".

The real four-node network was run until each node sent/received 30,000 messages of 15 words to/from the node three links away. This test was run several times with 
different network buffer sizes but with the user buffer and link speed set constant at 2000 words and $10 \mathrm{MHz}$ respectively. A few timers were added and the system appeared to reach stability almost immediately. The average time in the system is displayed in Table 1.

TABLE 1

Simulated Versus Real: Average Message Time in System (Seconds)

\begin{tabular}{ccccc}
$\begin{array}{c}\text { Buffer } \\
\text { Size }\end{array}$ & Real & Simulated & Difference & Relative Errer \\
\hline 36 & .00767 & .00492 & .00275 & .3585 \\
54 & .00748 & .00981 & -.00233 &. .3115 \\
150 & .03380 & .03900 & -.0052 & -.1538 \\
300 & .08300 & .08300 & .0000 & .0000 \\
500 & .14616 & .14633 & -.00017 & -.0012 \\
2000 & .60320 & .60330 & .00010 & -.0002
\end{tabular}

Intuitively, we could visualize the local user generator flooding the server with messages so the network buffer would be filled to capacity. Then, the user generator would be blocked and the server would be able to handle incoming messages by placing them in the contingency buffer. At some point, the server could reach a steady state of handing both incoming and local messages.

The simulation was then tested where each of the four nodes were sending/receiving continuously to the node three links away. The user buffer size and link speed were set to constants of 2000 words and $10 \mathrm{MHz}$ respectively. The test was run several times varying the network buffer size.

Each test was run for eight blocks, each representing 
one second of real time. The network was presumed to have been saturated with messages and reached steady state as the results for blocks three to eight were the same (as expected for constant input parameters). A comparison of the average message time in the system for both the real and simulated networks are shown in Table 1 and Eigure 5 .

\section{Average Message Time in System}

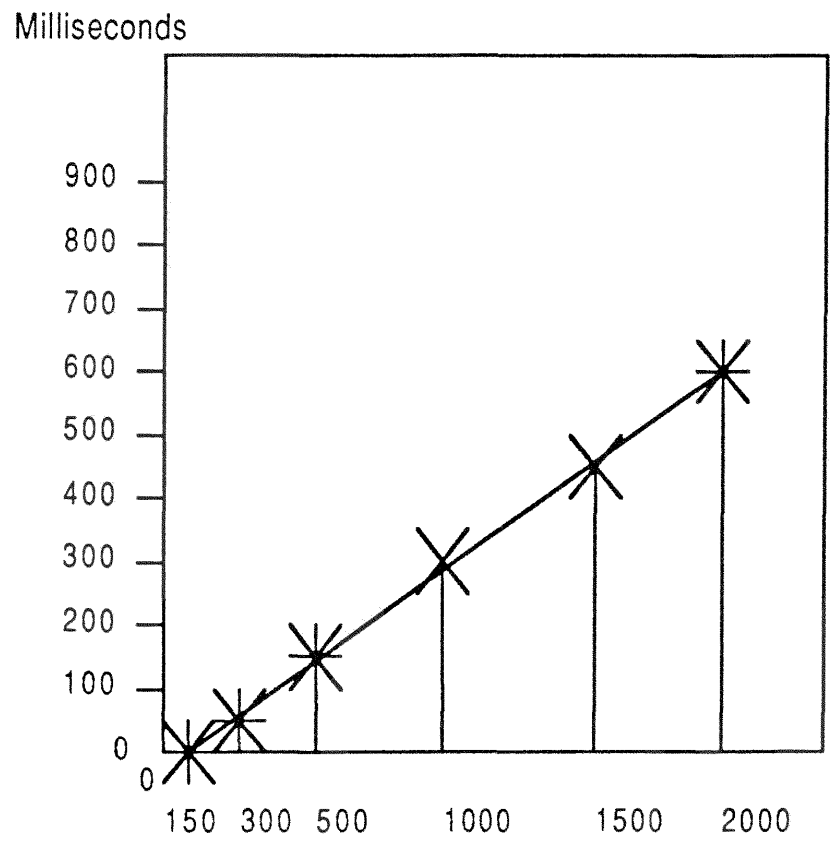

Network Buffer Size in Words

+ Real $\quad X$ Simulated

Figure 5: Simulated Versus Real: Message Time in System. 
The simulation was then run with uniformly distributed random message lengths between 1 and 31 words. Again, each node was sending messages across 3 links at $10 \mathrm{MHz}$. The user buffer was set to 2000 words. The simulation was set to run for 25 intervals each representing one-half second of real time.

The results are shown in Table 2 along with the $90 \%$ confidence interval which encapsulates the real network's average message time in the system (see Table 1). Note that network buffer sizes of 32 and 54 could not be tested because the maximum size of a message was 31 words and the network buffer was required to accommodate two maximum size messages (one for the contingency buffer, one for the network buffer).

\section{TABLE 2}

Average Time a Message Remains in a Four-Node Network With Random Message Length (Seconds)

\begin{tabular}{cccc} 
Network & $\begin{array}{l}\text { Average } \\
\text { Time in } \\
\text { Buffer }\end{array}$ & $\begin{array}{c}\text { Standard } \\
\text { System }\end{array}$ & $90 \%$ Confidence \\
Deviation & Interval \\
\hline 150 & .03185 & .00701 & .02033 TO .04337 \\
300 & .09867 & .01364 & .07623 TO .12111 \\
2000 & .79333 & .12100 & .59426 TO .99235
\end{tabular}

With several test runs and the results listed here, it was decided the model was valid. 


\section{CHAPTER 5}

\section{RESULTS}

In order to evaluate system performance, a well-defined, organized, and statistically sound testing method was required. Each test was run at least twice with different random number generator seeds in order to insure that no bias was added by the choice of seed. This chapter presents the major test results and findings of this research.

\subsection{SYSTEM PERFORMANCE UNDER DIFFERENT WORKLOADS}

When validating the model, it was noted that, message time in the system usually decreased as the buffer size decreased. However, real system performance was better at buffer size 54 than 36 . This indicated that smaller buffers increased system performance, but that at some point there was a cut-off, at which time performance slightly decreased. However, as determining the cut-off point was not part of this evaluation, tests in this section incorporated the fact that smaller buffers increased system performance, but did not seek to determine an "optimal" buffer size.

Testing was extremely time consuming (12 minutes to simulate one second of real time). Therefore, not all configurations could be thoroughly studied. Although the 
system had 23 parameters and could model numerous configurations, certain consistent parameters were used for all the tests described here. The network size was fixed with four processors. Because message lengths may vary, the tests used message lengths uniformly distributed between one and eleven words. The network and user buffers were kept relatively small ( 33 words--chosen to hold three maximum size messages). Lastly, as preliminary tests from the real and simulated networks indicated only slight improvement in system performance when the links were set at $20 \mathrm{MHz}$, it was decided to test with links set at $10 \mathrm{MHz}$.

Two workloads describing the message traffic were defined: heavy and moderate. The heavy load assumed the user application program continuously generated messages. The application program would spend only a few microseconds processing before generating its next message. The moderate workload had the application program run for a short while, thereby generating only a moderate number of messages.

There are four cases discussed in this section. Two for heavy workload and two for moderate workload. The heavy workloads used a constant of five microseconds for processing time between generating messages, while the moderate loads used a uniformly distributed processing time between zero and two milliseconds. Therefore, the heavy loads had one random number stream (message length), and the moderate loads had two (message length and processing time). 


\section{Workload Comparison \\ Average Message Time in System \\ Messages Travel 3 Links}

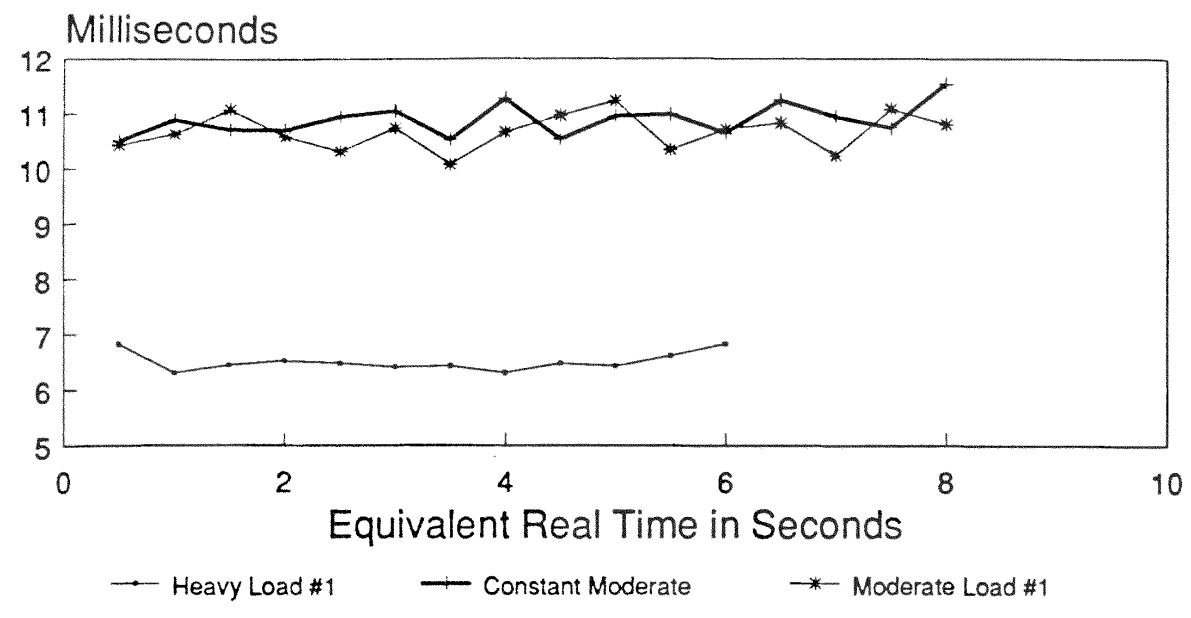

Messages (Uniform) 1 to 11 Words

Buffers@33 Words; Links@10 MHz

Figure 6: Workload Comparison.

Each load had a designated seed or seed pair used for each test. In order to compare workloads and to evaluate the effect of introducing the second random stream, the first heavy and moderate workloads used the same seed for message length. There was an additional run which used the same seed for message length but had a constant workload of one millisecond.

The simulation was run to model the network where each message destination was the previous node (message distance was three links/worst case analysis). Figure 6 displays the average message time in the system for the heavy, moderate, 
and moderate constant loads with the same message length seed. The randomness introduced by the process time can be seen along with the difference between workloads.

For each test case, several preliminary tests were run in order to determine when steady state was achieved. The simulation was run such that each node sent messages to the previous node. These preliminary tests were run for approximately 25 seconds of real time in block lengths equivalent to $1 / 4,1 / 2$, and one second. The "deleted moving average" for block lengths of $1 / 4$ and $1 / 2$ was computed and compared to the results of the one second block length. These data were examined to determine when steady state occurred and which block size was most appropriate.

It was found, that block length of $1 / 2$ second was less sensitive to random variation as the $1 / 4$ second block, and captured more information than the 1 second block. Thus, it was used for the block length of the following cases.

Each test workload was run for all possible message distances, for several seconds past the time determined as "steady state". The averages for message time in the system, following the decided steady state time, were then aggregated. Table 3 displays these aggregated averages and standard deviations. 
TABLE 3

Aggregate Average Time in System: All Loads (Miliseconds)

\begin{tabular}{lrrrr}
$\begin{array}{l}\text { Message } \\
\text { Distance }\end{array}$ & $\begin{array}{l}\text { Heavy } \\
\text { Load \#1 }\end{array}$ & $\begin{array}{l}\text { Heavy } \\
\text { Load \#2 }\end{array}$ & $\begin{array}{l}\text { Moderate } \\
\text { Load \#1 }\end{array}$ & \multicolumn{1}{c}{$\begin{array}{l}\text { Moderat } \\
\text { Load \#2 }\end{array}$} \\
1 Link & 1.755 & 1.766 & 4.031 & 4.003 \\
2 Links & 5.145 & 5.143 & 8.949 & 9.013 \\
3 Links & 6.413 & 6.554 & 10.777 & 11.048 \\
4 Links & 15.103 & 15.263 & 19.476 & 26.586
\end{tabular}

Eigures 7 through 10 display each $1 / 2$ block value for the different case workloads--from start-up through a couple of seconds at steady state. 


\section{Destination Length Comparison \\ Average Message Time in System \\ Moderate Load \#1}

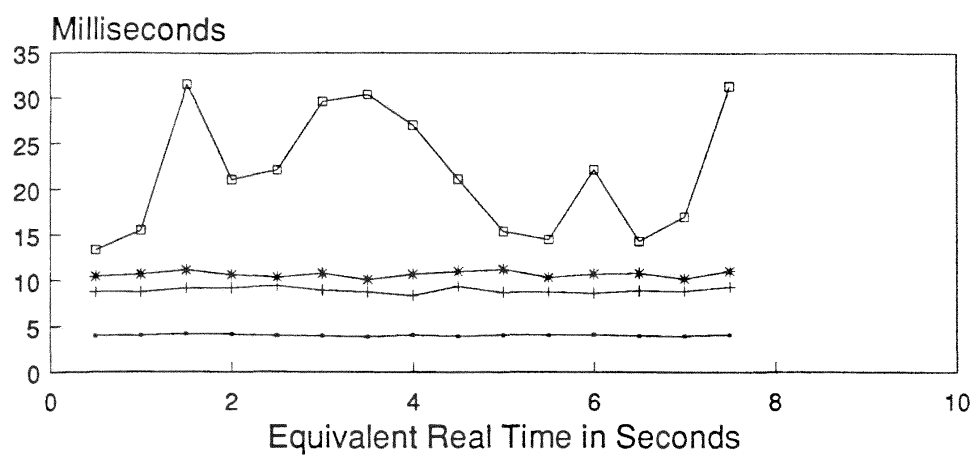

$\rightarrow 1$ Link $\rightarrow 2$ Links $\rightarrow 3$ Links $\rightarrow 4$ Links

Process Time (Uniform) $0-2$ millisec.

Messages (Uniform) 1 to 11 Words

Buffers@33 Words; Links@10 MHz

Figure 7: Average Time in System: Moderate Load \#1.

For all workloads, when message distance was one link (best case scenario), the time in the system was minimal. Clearly, no message had to compete with network messages to get into the network buffer. Each message was immediately placed in its network buffer, sent across the link, and was placed in the user buffer of the successor node, never really competing for space in any buffer.

Significant difference was found as soon as the messages had to travel more than one link. The competition for the network buffers can be seen in Figures 7 to 10 . 


\section{Destination Length Comparison \\ Average Message Time in System \\ Moderate Load \#2}

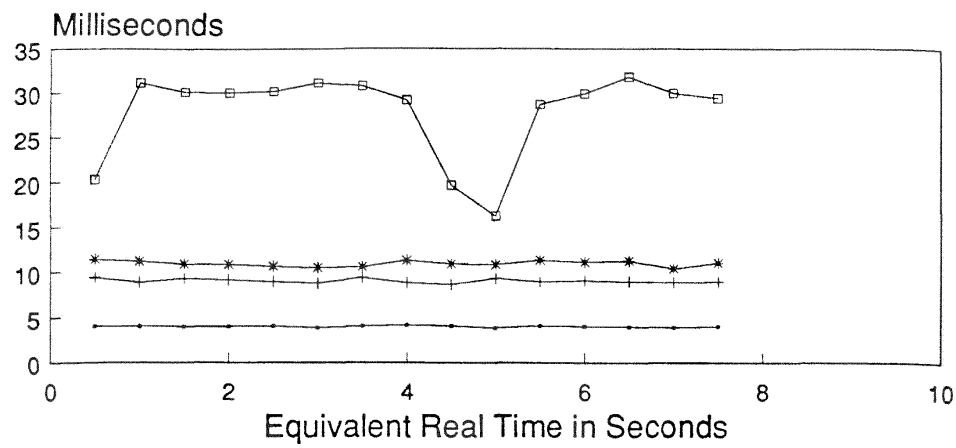

$\rightarrow 1$ Link $\rightarrow 2$ Links $\rightarrow 3$ Links $\rightarrow 4$ Links

Process Time (Uniform) 0 - 2 millisec.

Messages (Uniform) 1 to 11 Words

Buffers@33 Words; Links@10 MHz

Figure 8: Average Time in System: Moderate Load \#2.

Results of the two moderate workloads are displayed in Figures 7 and 8 . Comparable results were found.

There was a dramatic degradation in system performance when messages had to travel across four links. Messages were in circulation longer, competed for even more buffers, and were affected more by the randomness of the test than any other message distance. If a network were to be increased, and message distance were significant to the size of the network, projected system performance would degrade radically. 


\section{Destination Length Comparison Average Message Time in System Heavy Load \#1}

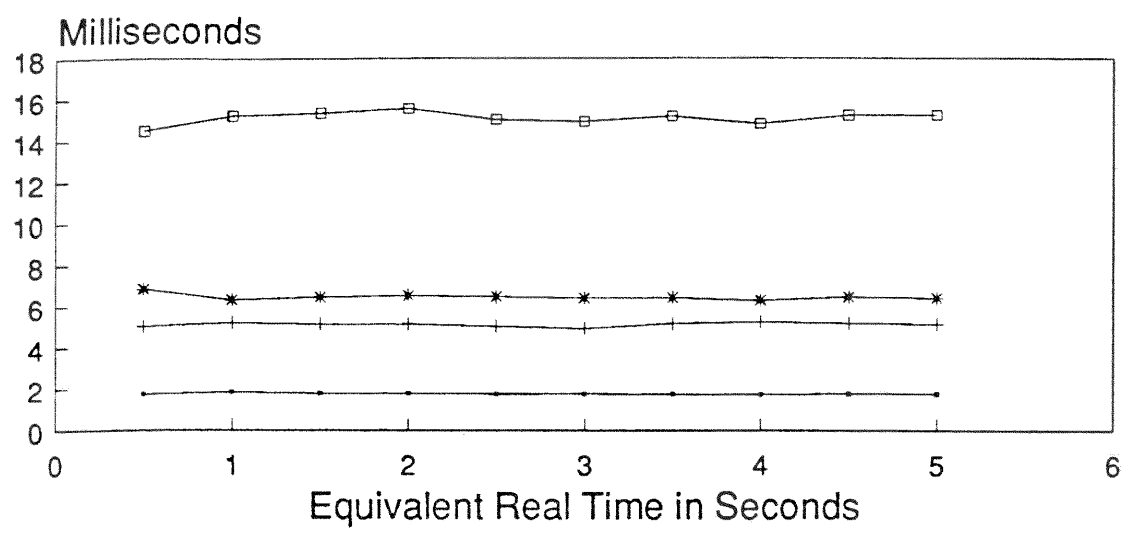

$\rightarrow 1$ Link +2 Links $\rightarrow 3$ Links $\rightarrow 4$ Links

Process Time (Constant) 5 microseconds

Messages (Uniform) 1 to 11 Words

Buffers@33 Words; Links@10 MHz

Figure 9: Average Time in System: Heavy Load \#1.

Results of the two heavy workload systems are displayed in Figures 9 and 10 . The results were consistent indicating the random seeds did not introduce a new bias. Because the application program was not really executing for any significant time, there was less time between the network processes running. As a result, message time in the system was decreased consistently across all message distances as compared with the moderate workloads. In fact, there was a minimum three millisecond increase for all message distances. 


\section{Destination Length Comparison \\ Average Message Time in System \\ Heavy Load \#2}

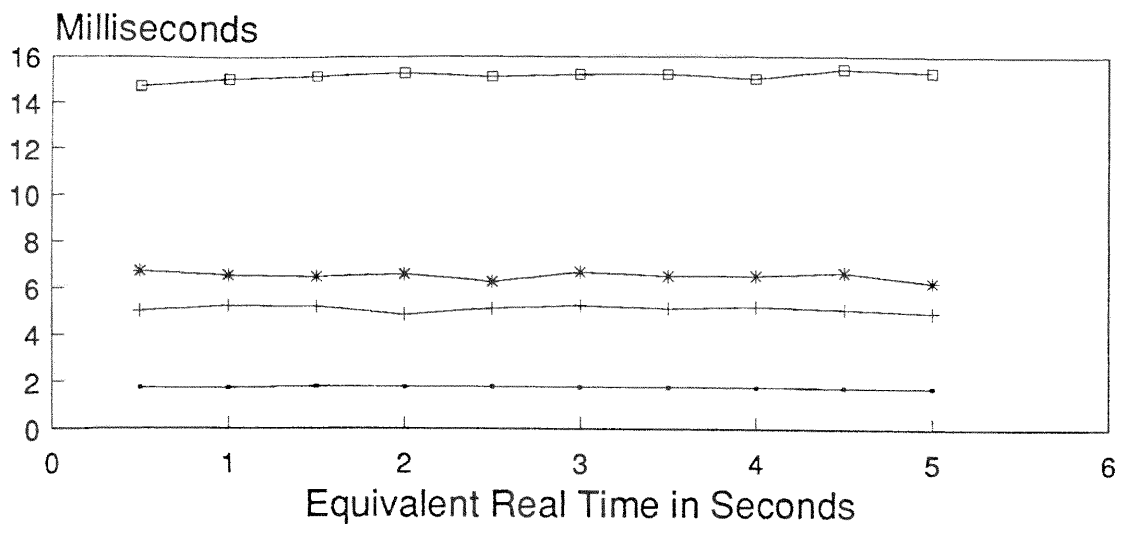

$\rightarrow 1$ Link +2 Links $\quad * 3$ Links $\rightarrow 4$ Links

Process Time (Constant) 5 microseconds

Messages (Uniform) 1 to 11 Words

Buffers@33 Words; Links@10 MHz

Figure 10: Average Time in System: Heavy Load \#2.

A five-node network was run with the message distance held constant at four links. The average message time in the system was found to be greater than with the four-node network with message distance of four links. Although all test cases were not run yet for the five-node network, the evidence indicated considerable degradation of system performance as the network size increased along with message distance.

\subsection{EFFECT OF BUFFER SIZES}

Several tests were run in order to determine the 
effect of changes made to the user and network buffer sizes. The random number generator used for message lengths (uniformly distributed between one and eleven words) was run with several different test seeds. Message distance was held constant to three links. Once the system reached steady state, the averages were aggregated and some are shown in Table 4. For these tests, the link speed was set at $10 \mathrm{MHz}$ and the network was run at heavy load.

TABLE 4

Effect of Buffer Size for Worst Case Scenario (Milliseconds)

Test seed \#37

\begin{tabular}{cccc}
$\begin{array}{l}\text { Network } \\
\text { Buffer }\end{array}$ & $\begin{array}{c}\text { User } \\
\text { Buffer }\end{array}$ & $\begin{array}{c}\text { Aggregated } \\
\text { Average }\end{array}$ & $\begin{array}{c}\text { Standard } \\
\text { Deviation }\end{array}$ \\
\hline 33 & 99 & 6.322 & 0.2045 \\
33 & 33 & 6.322 & 0.2045 \\
99 & 33 & 33.986 & 1.2344
\end{tabular}

Test seed \#83

\begin{tabular}{cccc}
$\begin{array}{l}\text { Network } \\
\text { Buffer }\end{array}$ & $\begin{array}{c}\text { User } \\
\text { Buffer }\end{array}$ & $\begin{array}{c}\text { Aggregated } \\
\text { Average }\end{array}$ & $\begin{array}{c}\text { Standard } \\
\text { Deviation }\end{array}$ \\
\hline 33 & 11 & 6.554 & 0.1716 \\
33 & 22 & 6.554 & 0.1716 \\
33 & 33 & 6.554 & 0.1716 \\
333 & 33 & 131.022 & 1.5609
\end{tabular}

* Messages (Uniform) 1 to 11 Words

* Message Distance (Constant) 3 Links

These results indicated that the user buffer was not a bottleneck. Thus, for the system at heavy load, the user buffer could be small. This would be useful for 
applications programs with large memory requirements. However, further research is needed in order to determine if this conclusion remains valid when the system is running at other workloads.

If the application program were required to communicate with only its successor node (best case), would it be more efficient to have larger buffers? Table 5 shows the results of the simulation program running at heavy load with message distance constant at one link. These results indicate, again, that smaller buffers improve system performance.

\section{TABLE 5}

Effect of Buffer Size for Best Case Scenario (Milliseconds)

\begin{tabular}{cccc}
$\begin{array}{c}\text { Network } \\
\text { Buffer }\end{array}$ & $\begin{array}{c}\text { User } \\
\text { Buffer }\end{array}$ & $\begin{array}{c}\text { Aggregated } \\
\text { Average }\end{array}$ & $\begin{array}{r}\text { Standard } \\
\text { Deviation }\end{array}$ \\
\hline 33 & 11 & 1.766 & 0.007 \\
33 & 33 & 1.766 & 0.007 \\
330 & 33 & 30.928 & 2.009 \\
330 & 330 & 30.928 & 2.009
\end{tabular}

* Message Distance (Constant) 1 Link

* System Running at Heavy Load \#2

Consideration should be given to the type of application program being run. For instance, if a program required significant computing time, larger buffers would minimize time spent waiting to send a message. The application program could generate a message, deposit it in the buffer, and continue processing. Although the message itself would remain in the system longer, the application program would not be blocked for a significant time. 


\section{CHAPTER 6 \\ CONCLUSIONS}

Both system throughput and average message time in system were strongly influenced by the size of the network buffer. When the buffer was large, the system could accommodate more messages. However, each message would have to remain in the system longer because it had to trickle through larger buffers.

The ring network studied was quite sensitive to message distance. As message destination length increased, system performance was radically degraded. Message time in the system increased because messages, not only had to travel further, but also had to also compete for space in each network buffer with the local messages being generated. Therefore, system performance is projected to decrease as both the size of the network and the message distance increase.

Lastly, special attention should be given to the type of application program to be executed on the system. If it is more important for an application to be able to execute than to minimize message time in the system, larger buffers should be considered. The network processes would be delayed because of the longer application run time.

In order to evaluate system performance of the 
Transputer network, a simulation model was designed. The model allowed investigation of workloads and conditions that would otherwise be at best difficult to monitor and analyze. With five processes running in parallel on each Transputer, the simulation attempted to model "chaos" in an organized and elegant fashion. 


\section{CHAPTER 7}

\section{FURTHER RESEARCH}

When message distance is increased the network performance is severely degraded. Thus, poor performance can be projected for large ring networks demanding intensive communication between processors. Therefore, if this project were extended, it is suggested to investigate throughput of other network topologies. Specifically, topologies which reduce the number of links a message must travel. One such topology is shown in Figure 11.

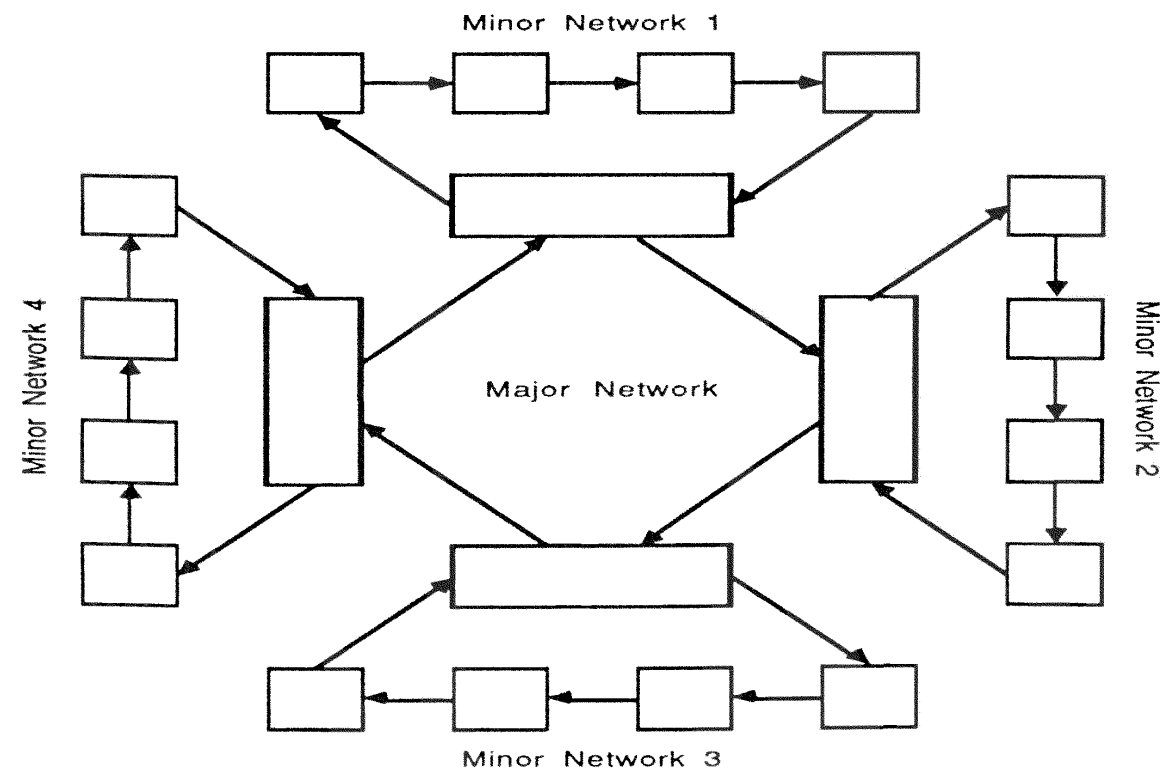

Figure 11: Alternate topology for large networks. 
Each "host" Transputer for a minor network would be responsible for sending its minor network messages onto the major network. Likewise, it would be responsible for receiving messages for its minor network from the major network. This particular "network of networks" could be simulated in a two-step process. First, statistics about the minor networks would be gathered. Second, the major network would be simulated by incorporating the minor network statistics.

It is clearly evident from the results obtained that the network buffer size effects message time in the system. System performance degrades when this buffer is increased slightly. Further research may find an "optimal" message to buffer size ratio for either a given number of processors, a given workload, or both. 
APPENDICES 


\section{APPENDIX A. THE STATE DIAGRAMS}

A description of each state and bound event for every server in the simulation is described in this Appendix. The symbols used are described in Eigure 12:

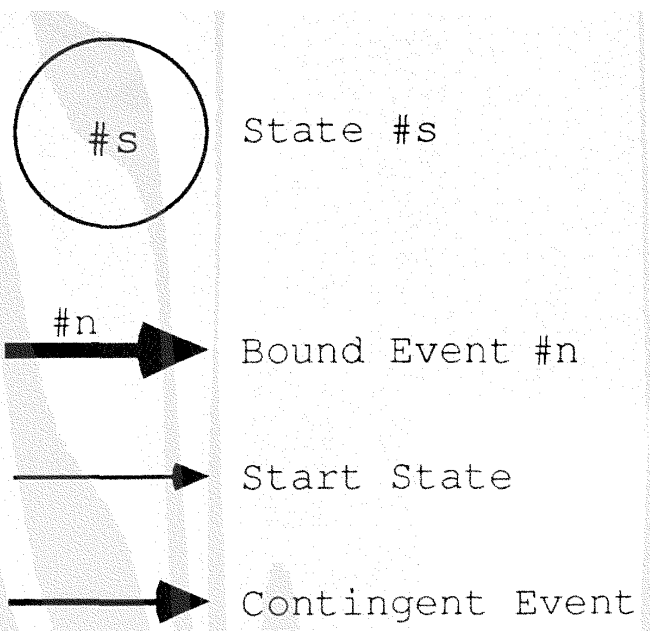

Figure 12: Summary of State Diagram Symbols. 


\section{The User Generator}

The states:

0. UG.Think -......-> running, thinking up messages

1. UG.Block -...-- $>$ blocked waiting to send a word

2. UG.Fill. Nbuff -..- filling network buffer (the server process is not running)

Bound Event Actions:

2. UG. Time. Out $\ldots-.->$ time out for running

3. UG. Xfer -......- time required to transfer a word of a message to the nbuff

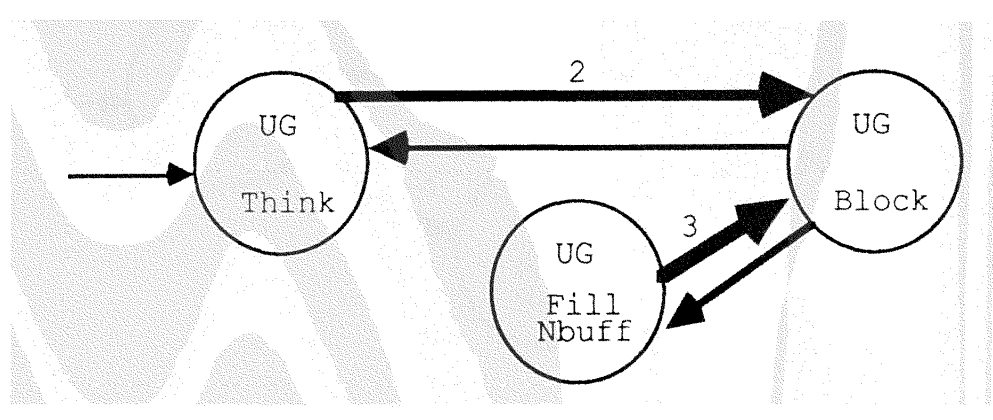

Figure 13: The User Generator State Diagram. 
The User Receiver

The States:

3. UR.Block. UF -...- $>$ waiting for UF to pass a word

4. UR.Block -...--> blocked waiting to read one word

5. UR. Read.Mail -.--> reading one word of a message

Bound Event Actions:

4. UR.Close.Mail -.--> read one word of a message

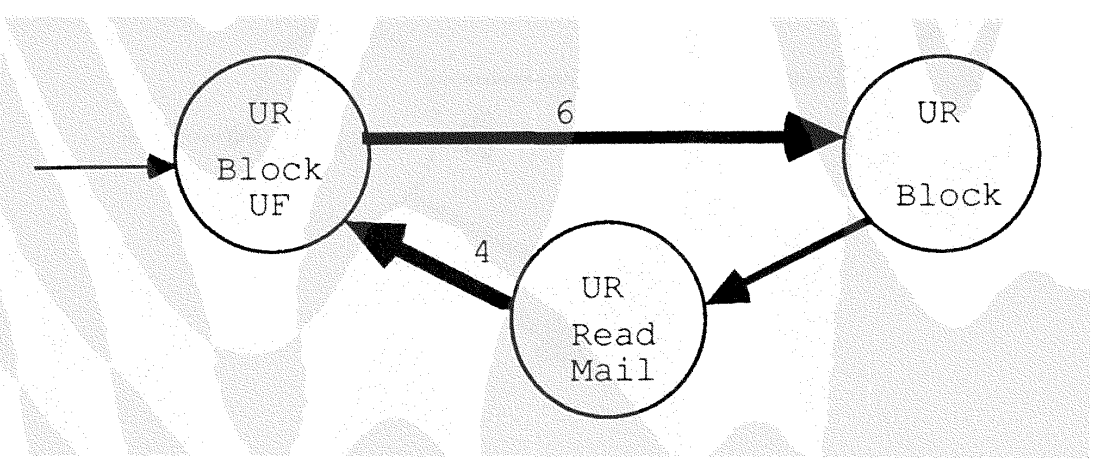

Figure 14: The User Receiver State Diagram. 


\section{The User Front}

\section{The states:}

6. UE.Block - - - - - b blocked, waiting to run

7. UE. Fill. Ubuff - - $\rightarrow$ placing word in user buffer

8. UE. Remove. Ubuff $\rightarrow$ removing word from user buffer

\section{Bound Event Actions:}

5. UF. Produce -----> place word in user buffer

6. UE. Consume -...- remove word from user buffer

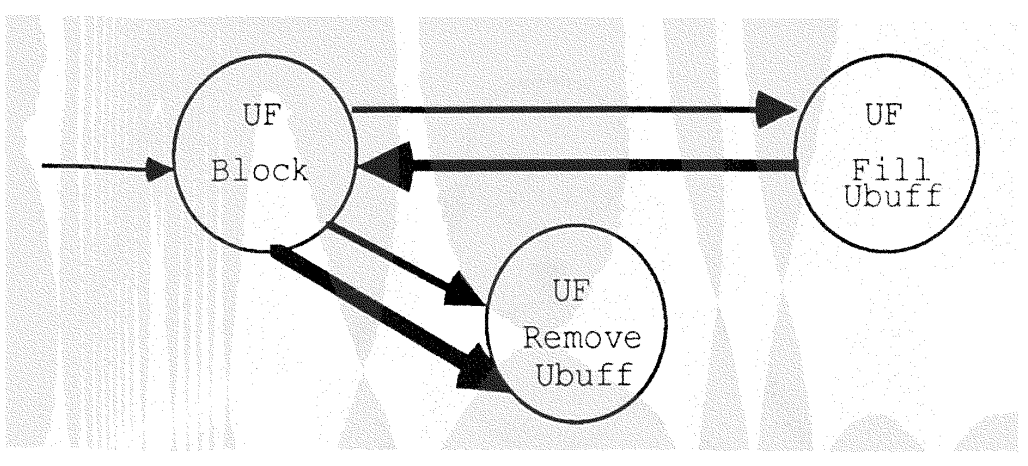

Eigure 15: The User Front State Diagram. 
The Network-In (Server)

\section{The states:}

9. NI.Sleep -......- nothing on link to get

10. NI.Block. Nbuff - - w waiting for room (net buffer)

11. NI.Block. Ubuff - -> waiting for room (user buffer)

12. NI.Block. UE -...-.> waiting for UF to run

13. NI.Wait. On.Link $\rightarrow$ waiting to get word on link

14. NI.Fill. Nbuff -..-> moving word (link to net buffer)

15. NI.Fill. Ubuff -.-> put word in user buffer via UF

\section{Bound Event Actions:}

7. NI.Get.Link -..--> a word arrived on the link

8. NI.Xfer -.....-- $\rightarrow$ word was moved (Iink-buffer)

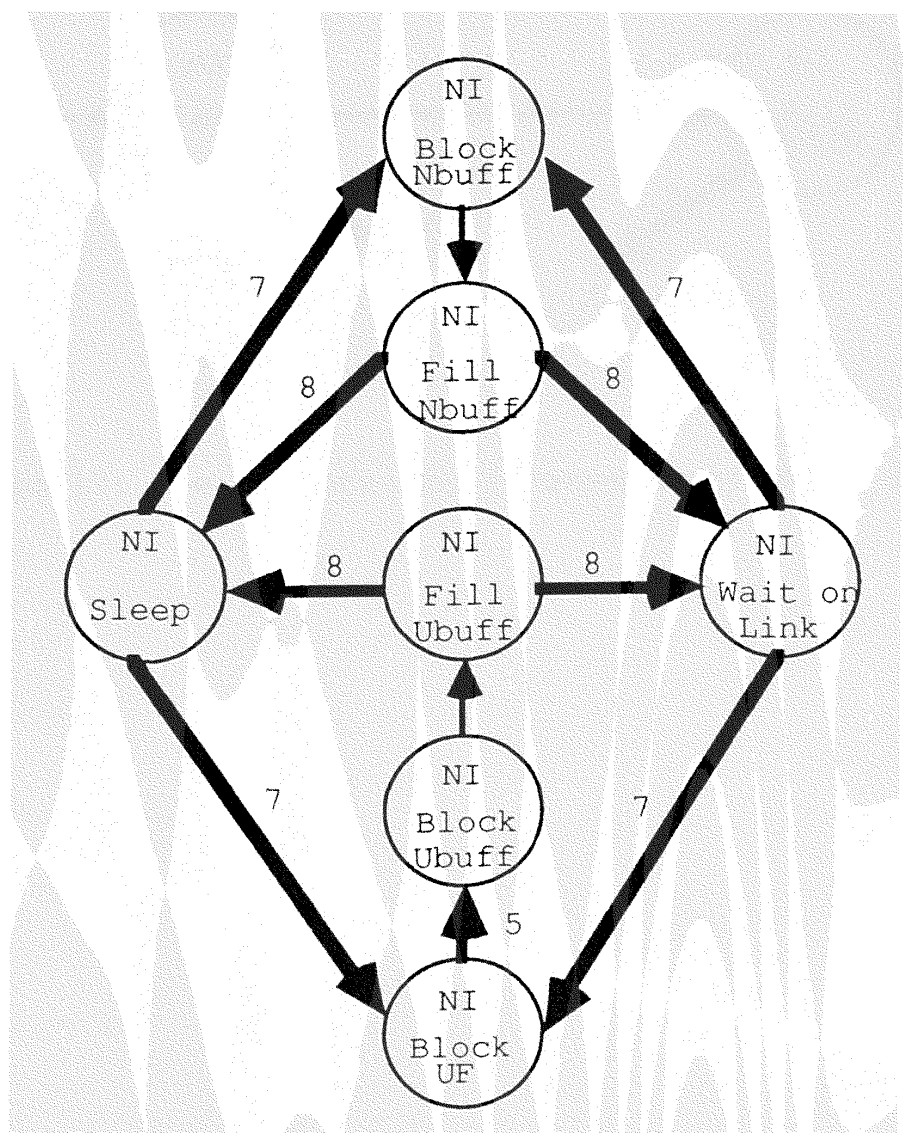

Figure 16: The Network-In (Server) State Diagram. 
The Network-Out (Transmitter)

\section{The states:}

16. No. Sleep - - - - - link to next node is empty

17. No.Busy -...-- $>$ Iink to next node is full

18. NO.Fill.Nlink - - > a word is being put on link

Bound Event Actions:

9. No.Xfer -.....-.

10. No. Received -.-- $\rightarrow$ the word on link was removed

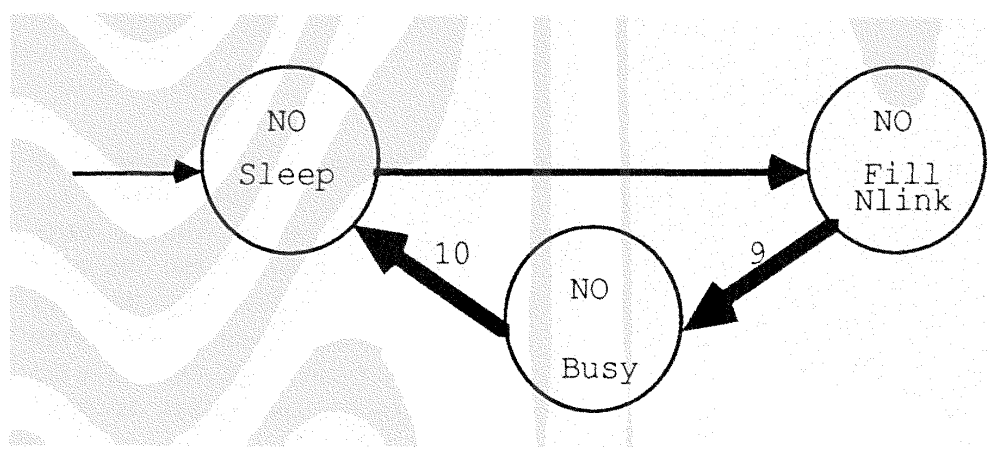

Figure 17: The Network-Out (Transmitter) State Diagram. 


\section{APPENDIX B. THE NETWORK COMMUNICATION CODE}

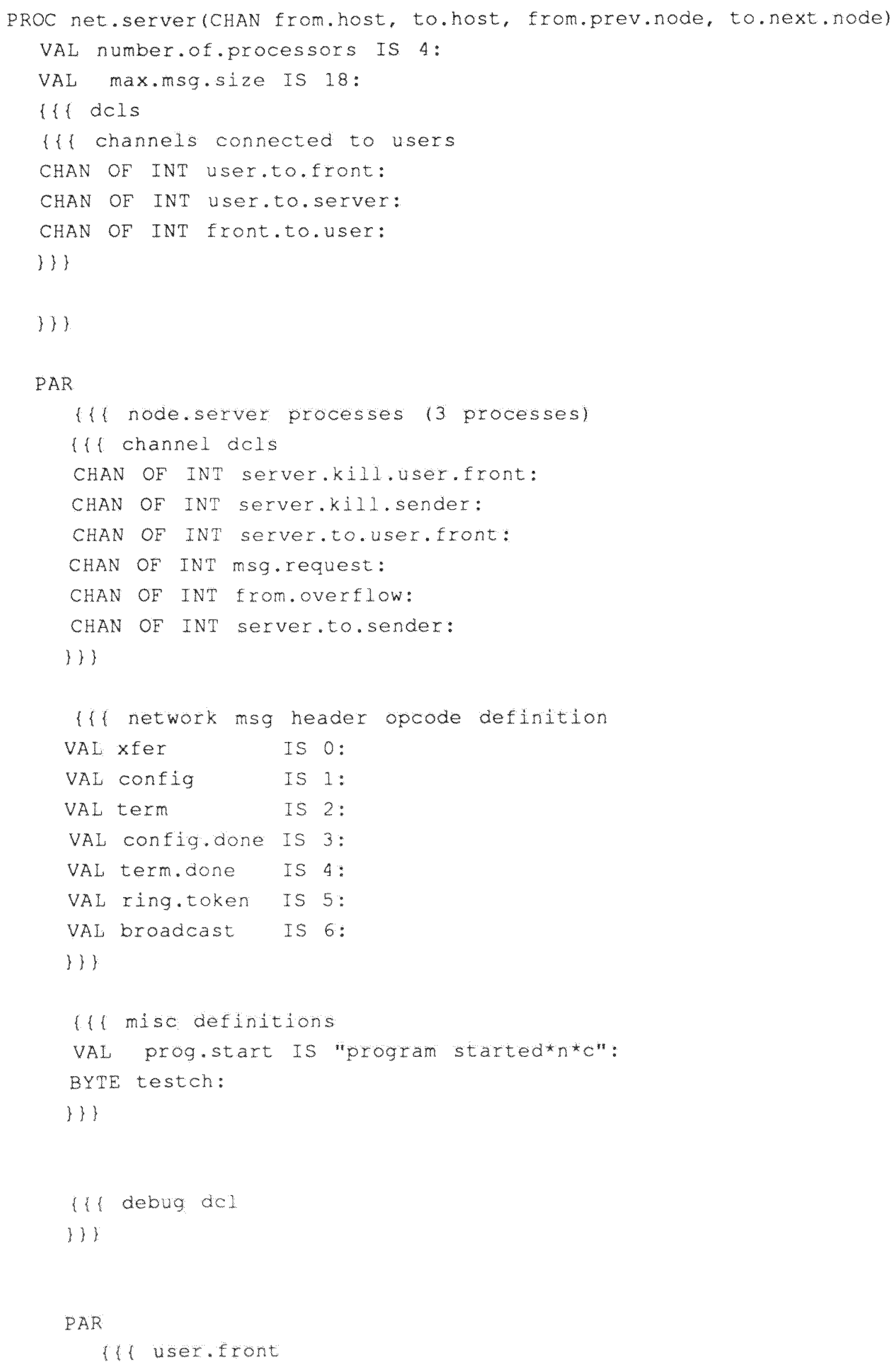




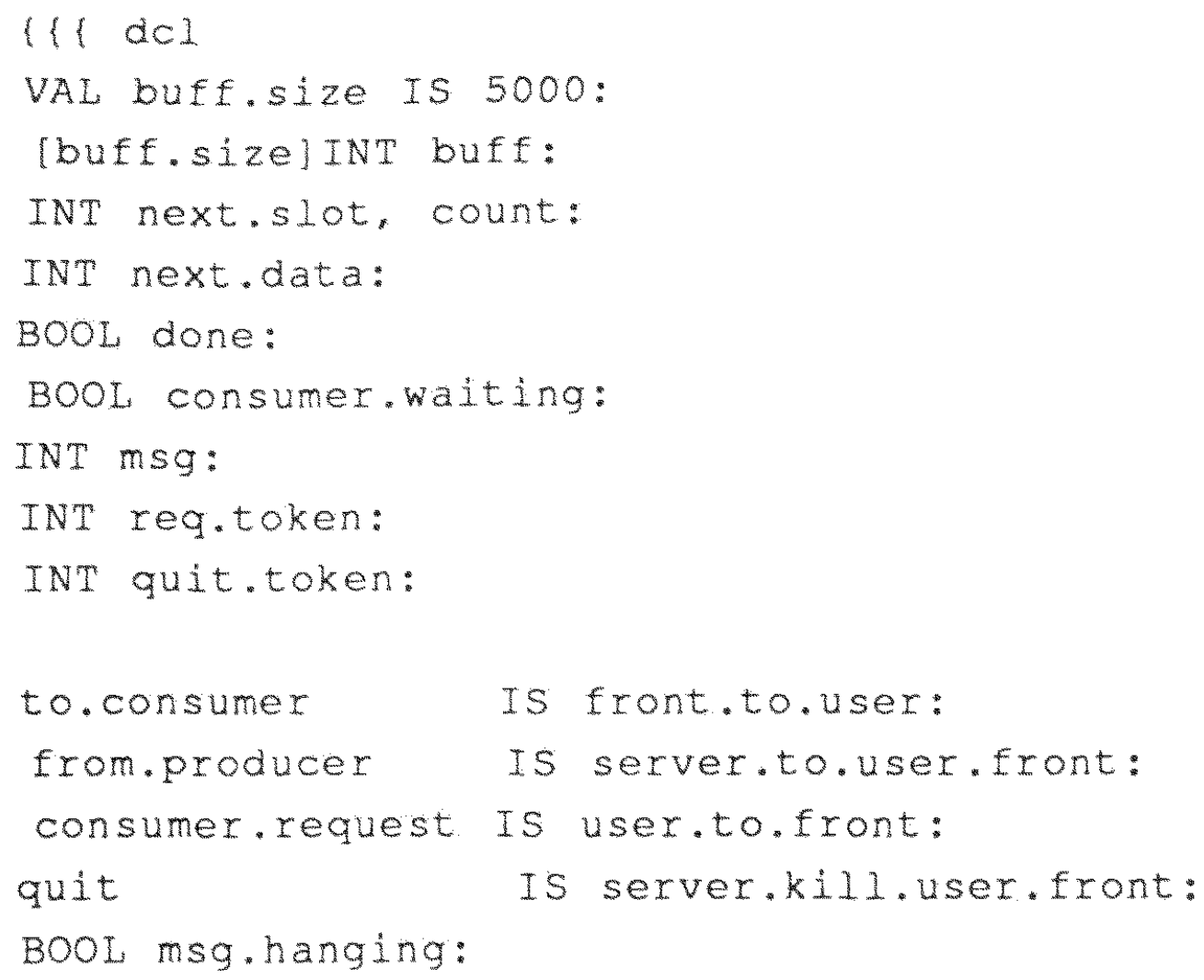


count: $=$ count +1

b)

TRUE

SEQ

msg, hanging: =TRUE

- ENDIE

) )

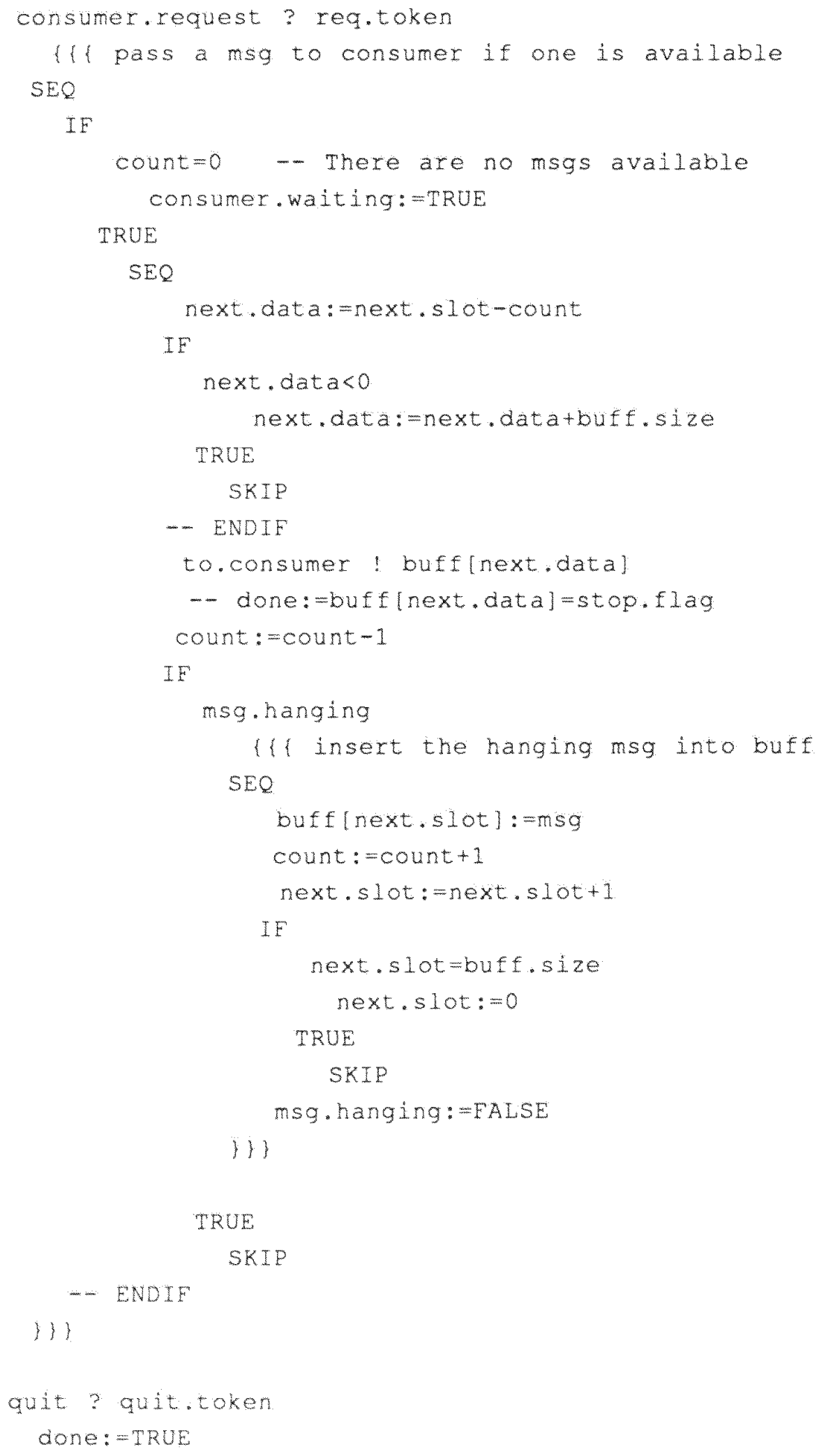


1)

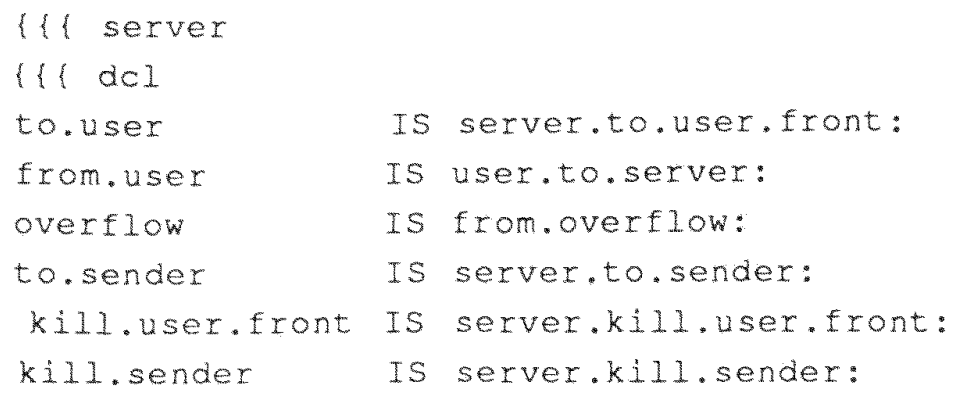




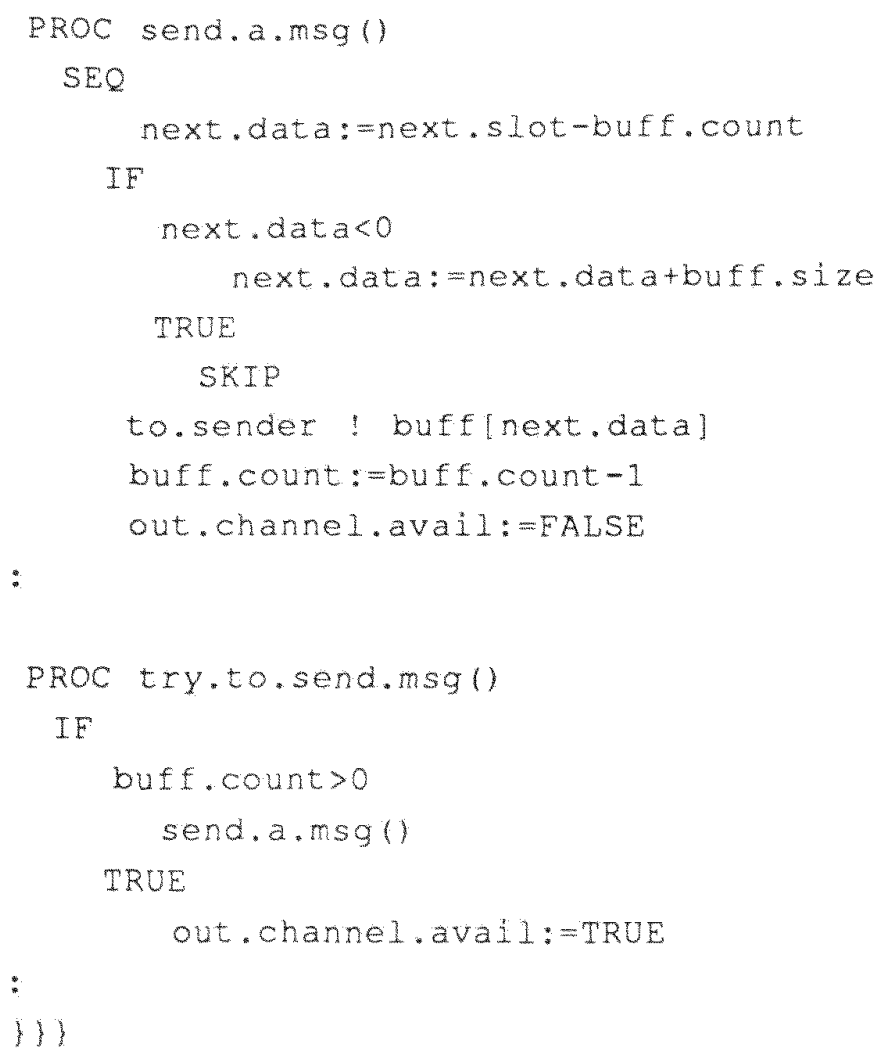


decode (msg, opcode, dest,msg.size)

IF

opcode=xfer

$\{1$

- -INT d:

SEQ

- d:=dummybuff $[3]$

IF

dest $=m y \cdot a d d r$

if transfer the whole msg to local user SEQ

WHILE msg.size>0

ALT

from.prev, node? msg

SEQ

to.user : $\mathrm{msg}$

msg.size: $=m s q \cdot s i z e-1$

msg.request ? req.token

try.to.send.msg()

1)

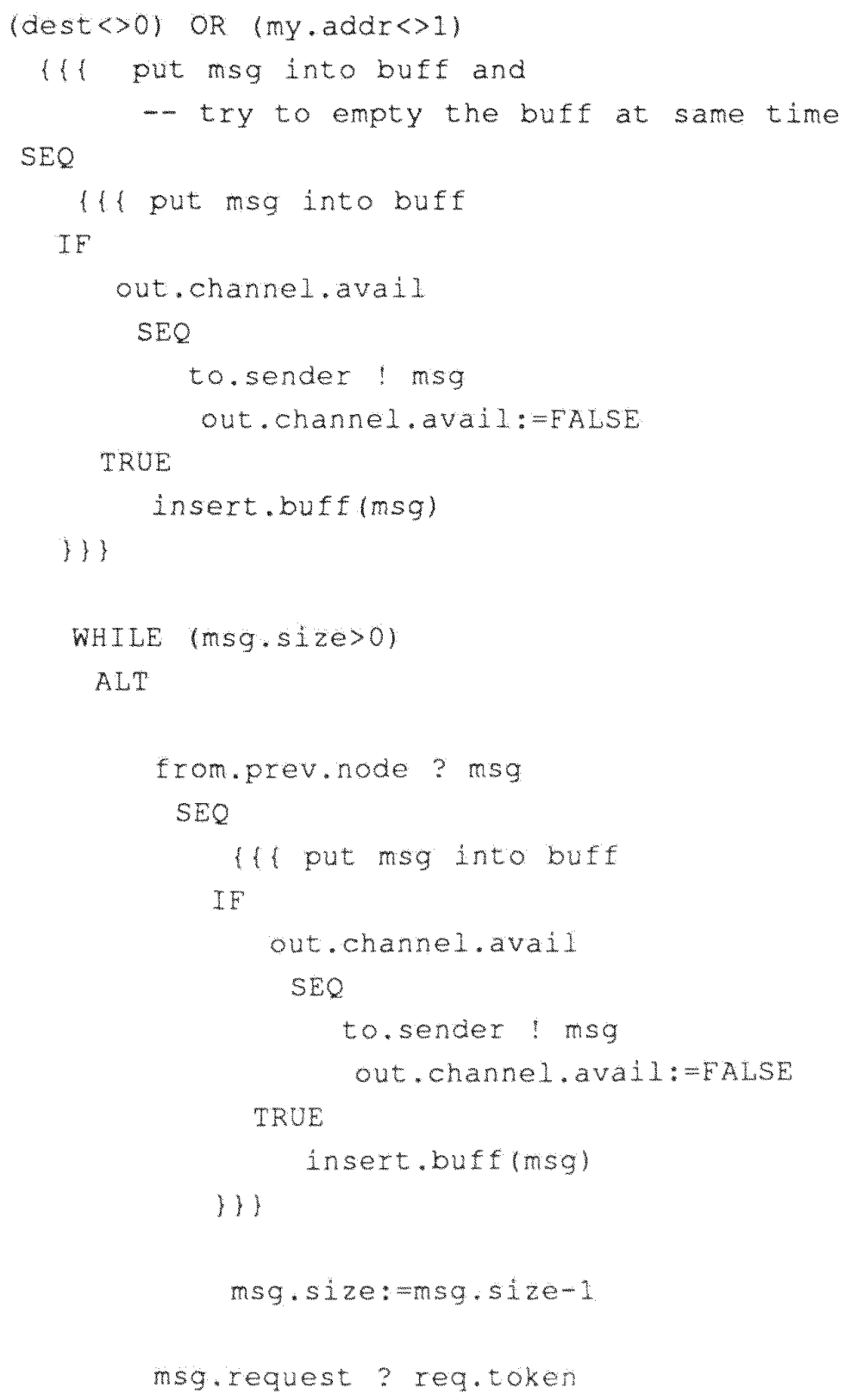


111

SEQ

IF

buef. count>0

send.a.msg()

TRUE

out, channel, avail:=TRUE

3)

-n try.to.send.msg()

3)

TRUE

$11 /$ pass the whole $\mathrm{msg}$ to host SEQ

to.host ! msg

WHILE msq. size>0

ALT

from.prev.node? msq

SEQ

to.host ! msg

msg.size: =msg. size-1

msg.request ? req.token

try.to.send.msg()

) )

b)

opcode=broadcast

11

SEQ

I

my. addr<number. of.processors

if put msg into buff

IF

out.channel. avail

SEQ

to.sender! msg

out. channel. avail:=FALSE

TRUE

insert.buff(msg)

1) !

TRUE

SKIP

WHILE (msg, size>0)

ALI

from.prev.node? $\mathrm{msg}$

SEQ

to.user ! msg

IF

my.addr<number. of.processors 
$11 /$ put $\mathrm{msg}$ into buff

IE

out. channel.avail

SEQ

to. sender ! msg

out , channel. ava11:=FALSE

TRUE

insert.buff (msg)

1)

TRUE

SKIP

msg.size:=msg.size-1

msq.request? req.token

I I

SEQ

IF

buff. count $>0$

send.a.msg()

TRUE

out. channel.ava11:=TRUE

) H

-- try.to.send.msg()

())

opcode $=$ contig

11

SEQ

IF

NOT configured

SEQ

configured: =TRUE

my. addr:=dest

dest: $=$ dest +1

make. net. header (config, dest, 0 , msg. header)

wait. for out channel()

to.sender ! msg, header

TRUE

SEQ

make.net. header (config.done, dest, 0 , msg.header)

to.host ! msg.header

1) )

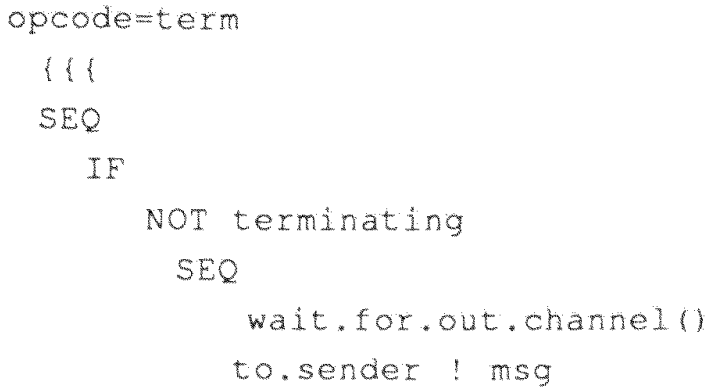

TRUE 
SEQ

make.net.header (term. done, 0, 0, msg.header)

to.host ! msg.header

kill,user.front! kili.token

kill.sender! kill.token

run:=FALSE

3) )

TRUE

SKIP

$3\}$

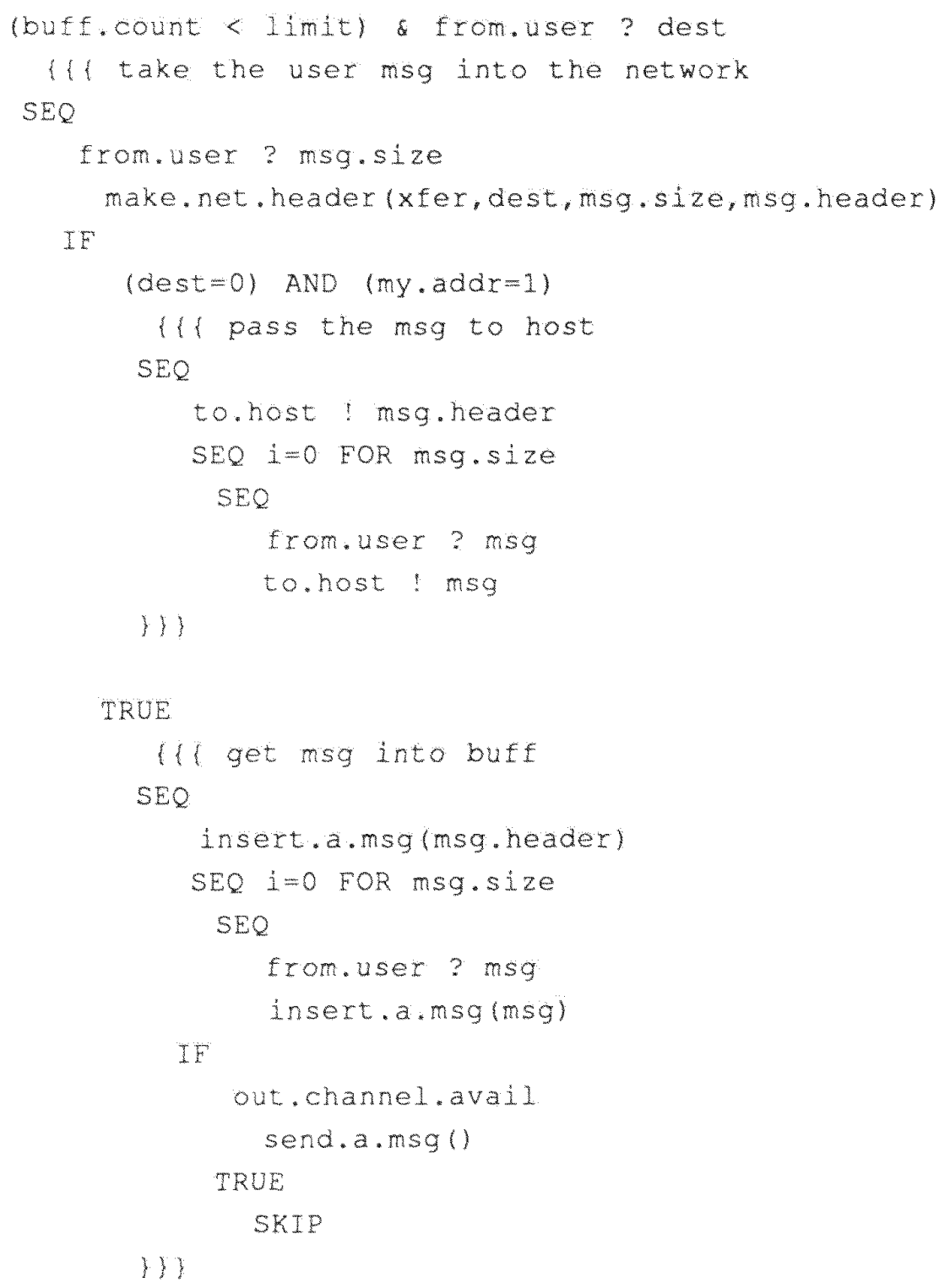




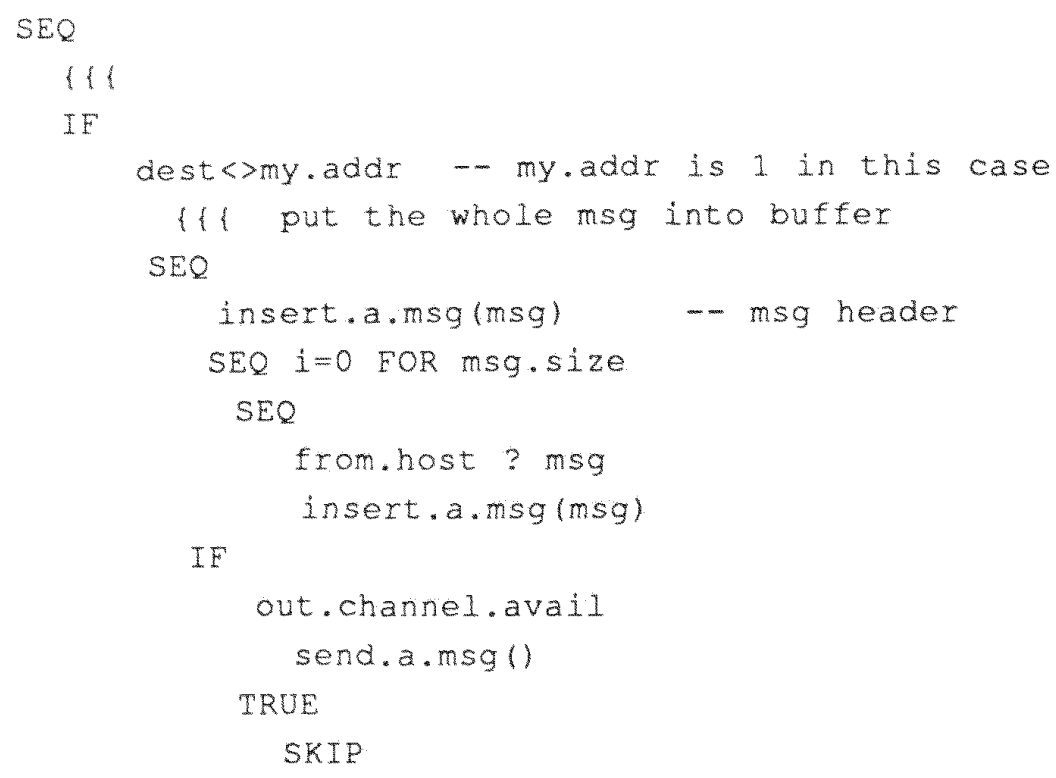

3)

opcode=broadcast

is put the whole msg into buffer

SEQ

insert.a.msg(msg)

- msg header

SEQ $1=0$ FOR msg.size

$S E Q$

from.host? msg

insert,a.msg(msg)

to.user! msq

IF

out. channel. avali

send.a.msg()

TRUE

SKIP

1)

opcode=config

II

SEQ

$m y \cdot a d d r:=d e s t$

dest: $=$ dest +1

make.net, header (config, dest, o,msq. header)

wait. for out. channel()

to.sender ! msgheader 
configured: =TRUE

i)

opcode=term

111

SEQ

terminating:=TRUE

wait. for out. channel)

to.sender ! msg

3) \}

TRUE

SKIP

)1)

1)

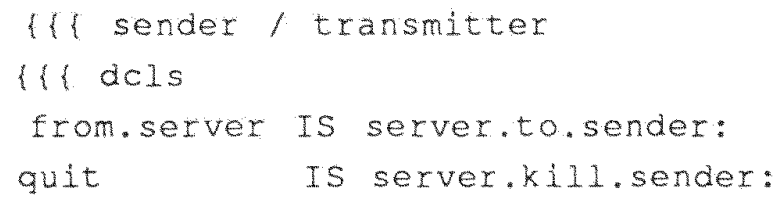

Bool run:

INT req.token, quit.token:

INT msg:

))

SEQ

run: = TRUE

WHILE TRUE

SEQ

msg.request : req.token

from.server? msg

to.next.node ! msg

i $\{$ COMMENT

)! 3

))

(T) channel dol for user

get.msq

Is front.to.user:

request.msg Is user.to.front:

send.msg

Is user.to.server:

b)

i)

( ( IF usernode.tsr (2 processes) *usernode.tsr

il 1 user msg header function code definitions

VAL data

IS 0:

VAL config

IS 1: 


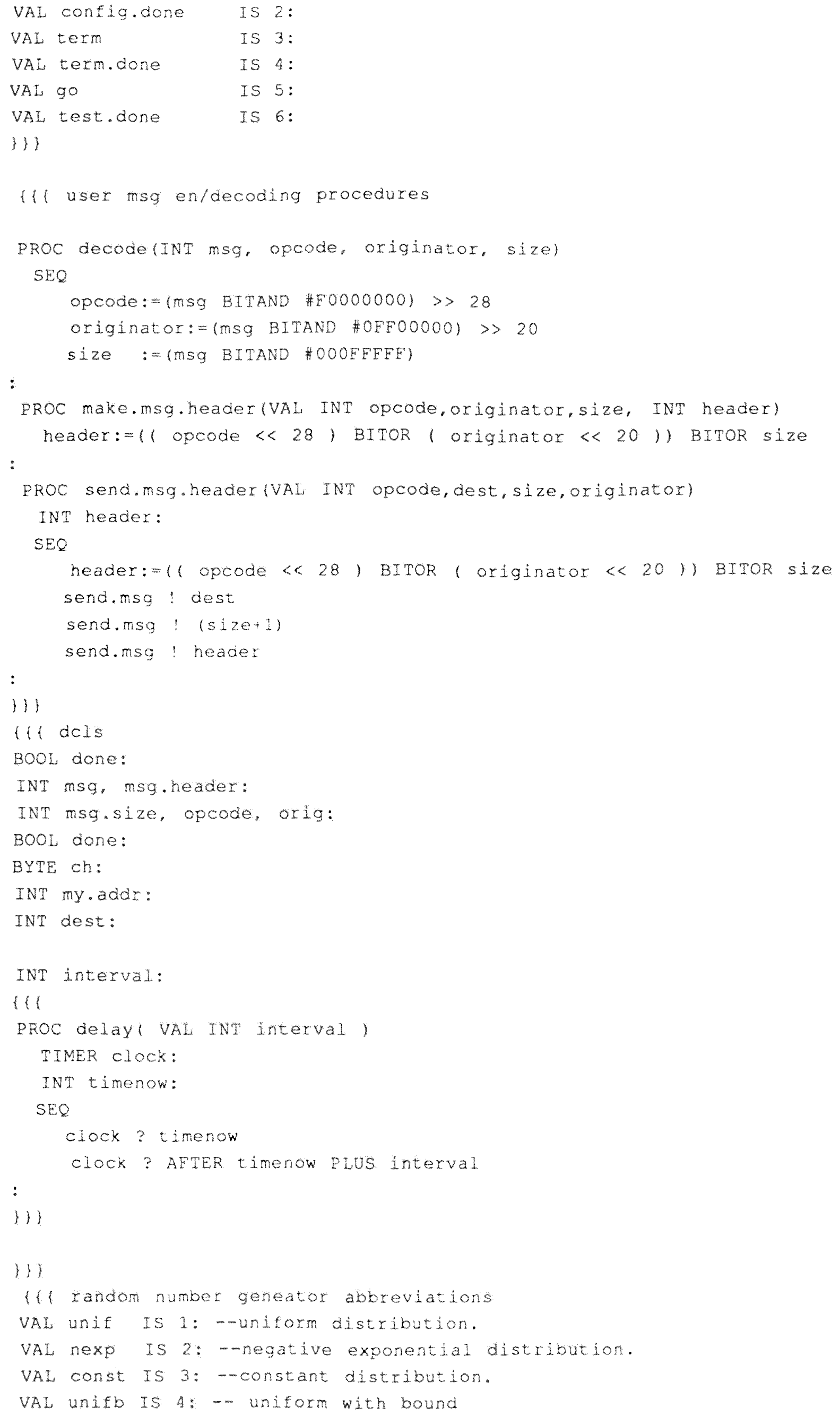




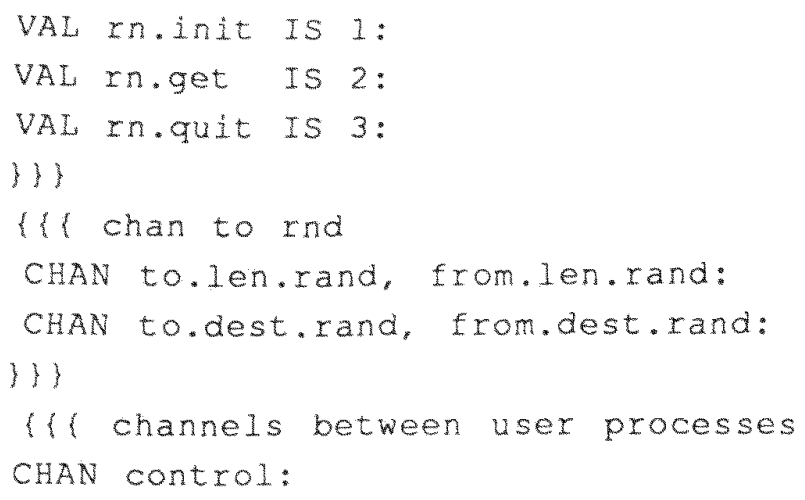


) )

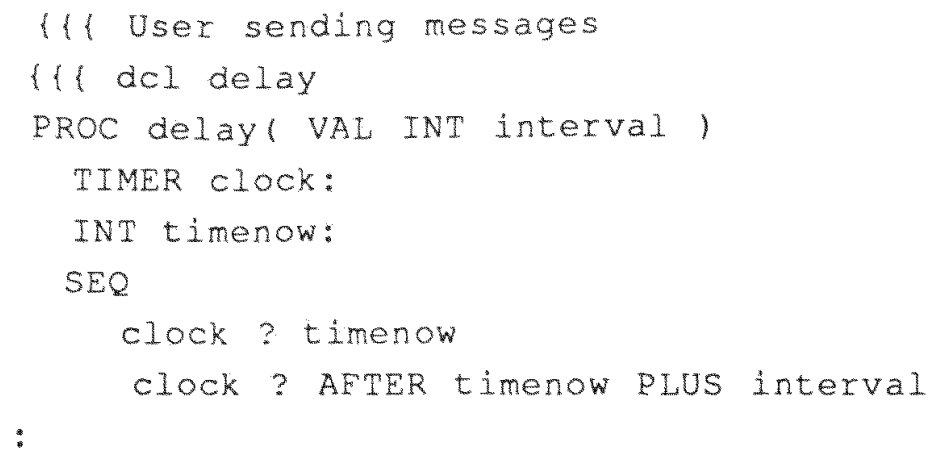


send.msg : my.addr

IF

(i $(1000)=0$

SEQ

send.msg. header (data, 0,1, my.addr)

send.msg ! my.addr

If

$i=20000$

clock? start,time

$\dot{i}=22000$

clock? finish.time

TRUE

SKIP

TRUE

SKIP

1)

send.msg.header (data, 0,8, my. addr)

send.msg ! my.addr

send.msg ! (finish.time-start.time)

SEQ $i=1$ FOR 6

send.msg : ( INT $=0)-($ INT 10$)$

send.msg. header (test. done, 0,0, my.addr)

control ? msg

send.msg.header (term.done, 0, 0, my.addr)

) )

1) 


\section{APPENDIX C. THE SIMULATION CODE}

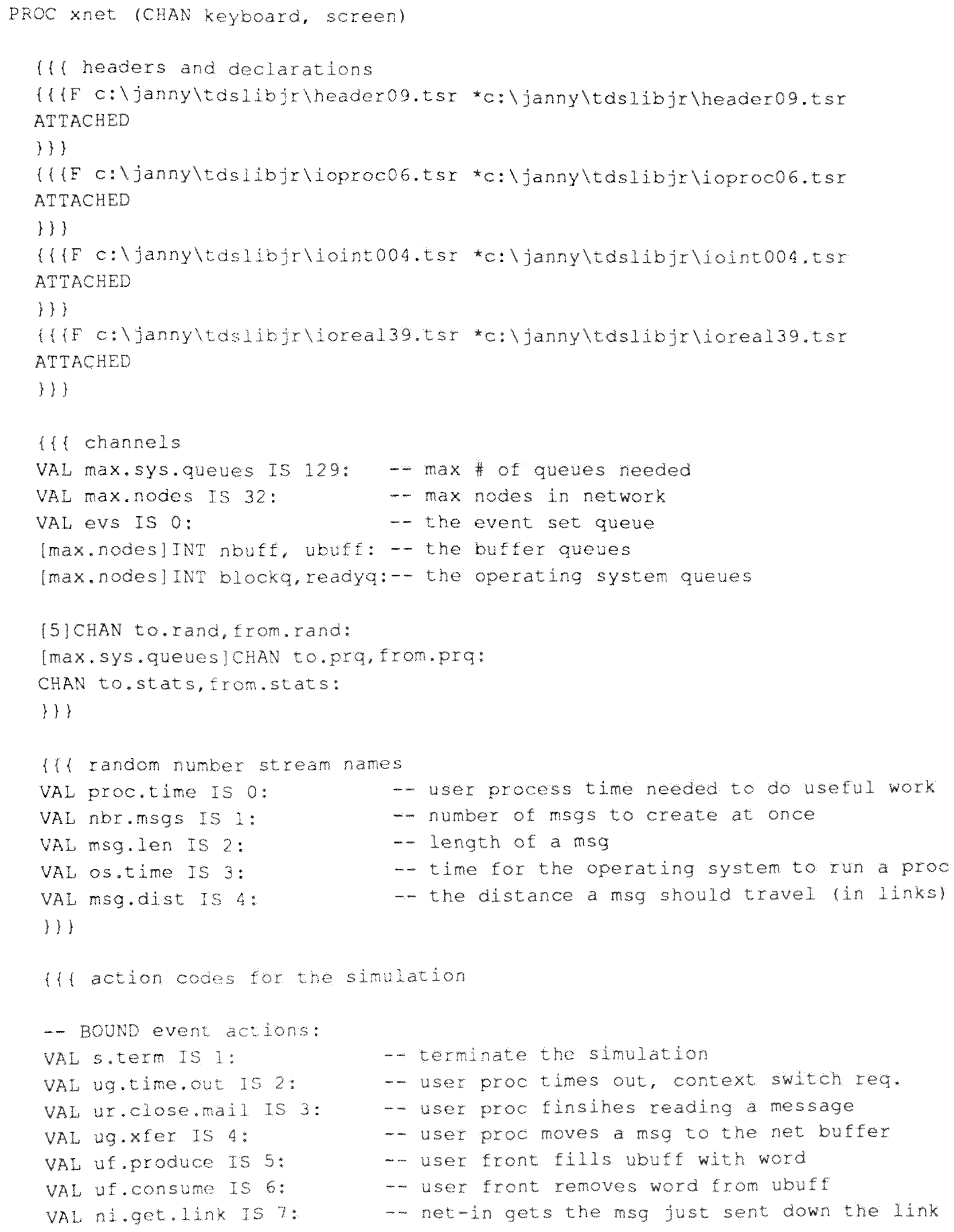




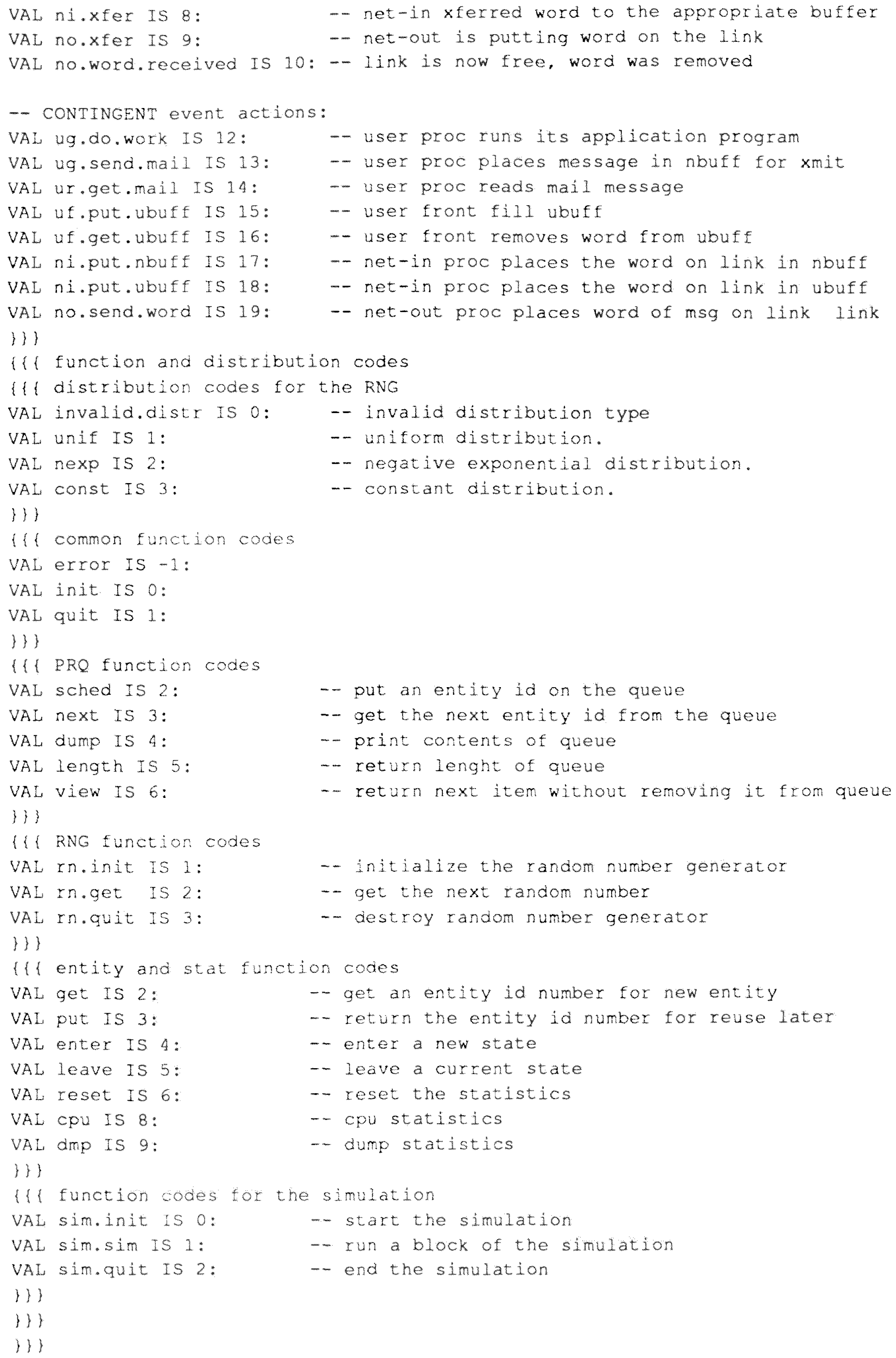




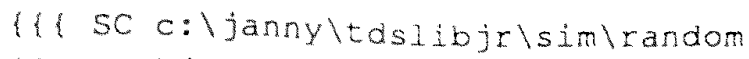

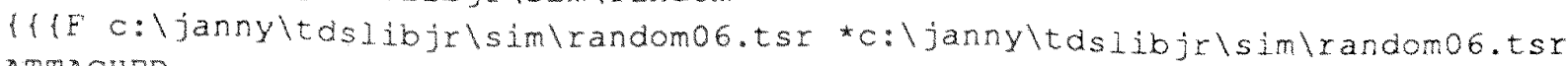
ATTACHED

3)

3)

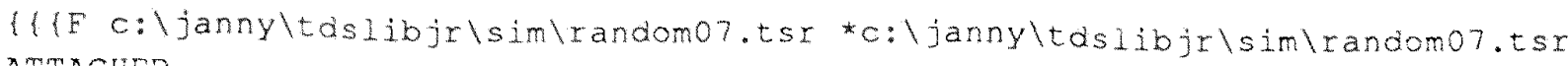
ATTACHED

) )

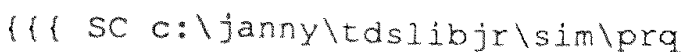

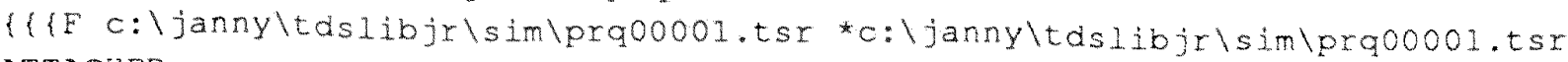
ATTACHED

) )

) 1

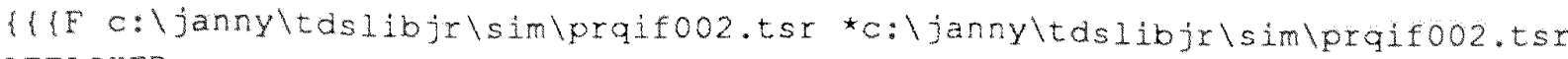
ATTACHED

) \})

\{l SC c: Jannyltasibjr\simistats

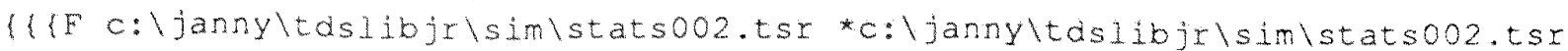
ATTACHED

H)

) )

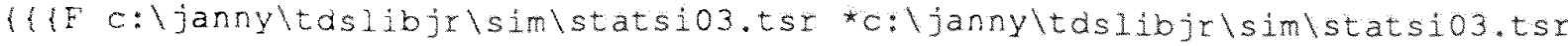
ATTACHED

1) ?

[23] INT params:

$\{1$ parameter map

max.msg.len Is params[0]:

- max length of a message

max.nbuff Is params $[2]$ :

-- max number of words nbuff can hold

max.ubuff Is params [3]:

-- max number of words ubuff can hold

n.blocks is params [4]:

- number of blocks to run

block. Ien Is params[5]:

-- length of each block

trace is params (6):

- values of the trace

seed.msg.dist Is params [1]:

- seed for msg dist -- \# links a msg travels

seed.proc.time Is params [7]:

- seed for the process time between gen msgs

seed.gen.msgs Is params [8]:

- seed for the number of msgs being created

seed.msg.Ien IS params [9]:

-- seed for the length of $\mathrm{msg}$ being created

seed.ostime IS params [10):

- seed for the ostime (op sys delay)

mean.proc.time Is params [11]:

mean.ostime is params [12]:

- mean process time between generating msgs

mean.gen.msgs is params [13]:

- mean sleep time for the receiver

mean.msq. Ien IS params 14 1:

- mean number of messages created at once

- mean length of a generated message

mean.msg.dist is params[1b:

-- the mean number of links a msg travels

cwxmit. Is params [16]:

- the speed of the link

n. nodes is params [17):

-- the number of nodes in the system

distr.proc.time IS params[18]:

-- distr type for local user process

distr.gen.msgs is params [19]:

- distr type for \# msgs to generate at once

distr.msg.ien is params[20]: - distr type for message length 
distr.ostime Is params [21]: - - distr type for operating system delay

distr.msg.dist Is params[22]: - distr type for \# links a msg should travel

I)

PROC xnetsim(VAL INT opus, TNT clock)

\& 1 run the simulation

il states of the system

VAL ug.think IS 0 :

- user proc is thinking/processing

VAL ug.block IS 1 :

- user proc blocked waiting to read/send mail

VAL Ug. fil1. nbuff IS 2 :

- user proc is filling nouff with a msg

VAL ur.block, uf IS 3:

VAL ur.block IS 4:

- user proc is waiting for user front to run

VAL ur.read.mail IS 5:

-- user proc is blocked to read mail

- user proc is reading a mail $\mathrm{msg}$

VAL UE. block IS 6 :

- user front process is not doing anything

VAL Uf. fill, ubuff IS 7 :

- user front process filling ubuff

VAL uf. remove. ubuff IS 8 :

- user front process is removing from ubuff

VAL ni.sleep Is 9:

- net-in is sleeping, nothing on link to get

VAL ni.block. nbuff IS 10:

- net-in is blocked waiting for the nbuff

VAL ni.block, ubuft IS 11:

- net-in is blocked wating for the ubuff

VAL ni.block. Uf IS 12 :

- net-in is waiting for user front to run

VAL ni.wait.on.link IS 13:

- net-in is waiting to receive a word on Iink

VAL ni.fill. nbuff IS 14:

-- net-in moves the msg to nbuff from the link

VAL ni.fill, ubuff IS 15 :

-- net-in moves the msg to local ubuff

VAL no. sleep IS 16:

- net-out in ldle state (link is not busy)

VAL no.busy IS 17 :

- net-out in xmit state (link is busy)

VAL no.fili.nlink is 18:

-- net-out is filling the link with a word

VAL msg.traffic IS 19:

) )

1/ states of the Iink

VAL link. no.msg is o:

VAL link, head.msg IS 1:

VAL link. word.msg Is 2 :

3)

111 constants for testing

VAL max.proc.time Is 3000:

- msg header is in the system

VAL U, read header IS 45:

VAL U.read.word is 16:

VAL u.word.gen IS 10:

VAL u header.gen Is 62 :

VAL $u$.put. h.nbuef IS 61 :

VAL U. put. W. nbufe IS 19:

- Link is free

- Iink holds the header of the $\mathrm{msg}$

- link holds a word of the msg

VAL uf.get IS 40:

VAL Uf. PUT IS 28:

- max proc time before time slice

- time to read header (t 10 for clock)

-- time to read one word of a msg

- time to generate one word of $\mathrm{msg}$

- time to generate the header, (+10 clock)

VAL ni.put.h.nbuff IS 47:

- time to put header in nbuff

-. time to put word in nbuff.

- "time for user front to get next word

- - time for user front to put next word

-- time to place header in nbuft 


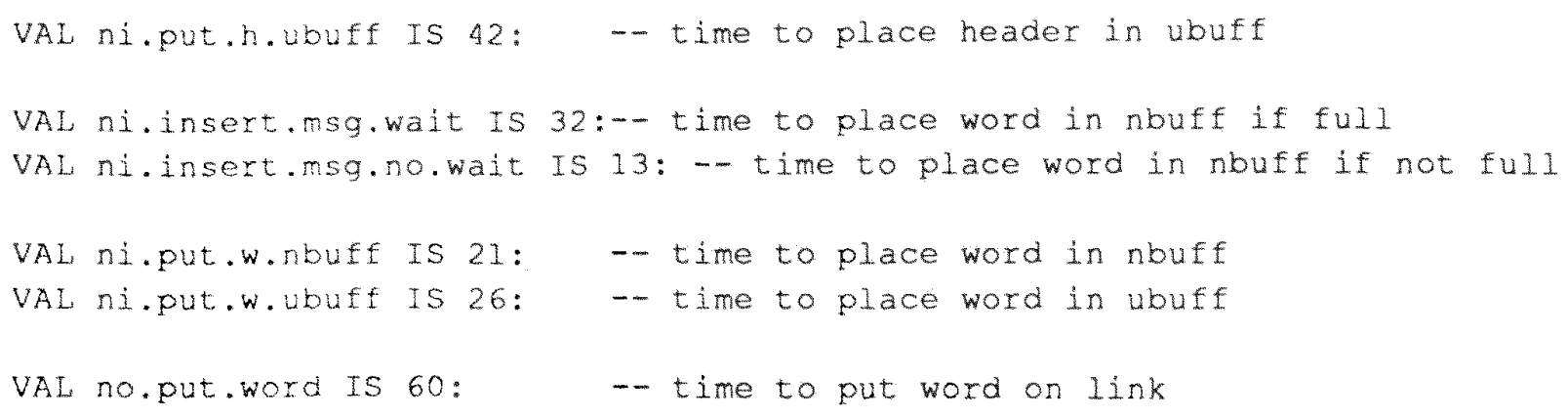


INT gen.msg.can.fit:

INT send.nbr.msgs:

INT think:

INT read,time, send, time:

INI $i, j:$

INT act, node, nor.words:

INT ch:

INT stime, newtime:

TIMER realclock:

INT etimer, stimer, ftimer:

REAL32 durance:

INT clock:

BOOL run:

) )

$\{1$ entity control

il entity object parameters

VAL maxent IS 20000:

VAL num. of. fields IS 5:

VAL maxstate IS 14:

VAL maxatr is 4 :
- max length of nbuff so that 9 can add msg

-- temp var to get random number

- temp var used to get random think time

- time to read or send a word of a $\mathrm{msg}$

- loop control vars

- holds values for an entity

- used to hold clock time

- used for timing

-- more timers

-- THE FIELDS OF THE STRUCTURE ENTTTY:

VAL action IS O:

VAL link IS 1:

-- the bound event action id

VAL node. Id IS 2 :

- used to link entitites

VAL n.woras IS 3 :

- the node associated with the entity

VAL fdest IS 4 :

- number of words in the msg / with header

- holds the node to recelve the $\mathrm{msg}$

-- THE STRUCTURE ENTITY:

[maxent] [num. of.fields]INI entity: -- the storage for the entities

3)

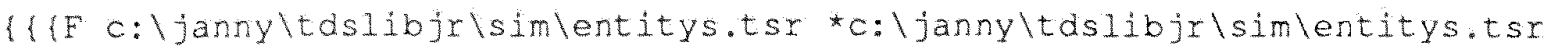
ATTACHED

) )

1) )

$S E Q$

IF

opus $=\operatorname{sim} \cdot \operatorname{in} i t$

if initialize the model

SEQ

(U) initialize the entity object

ent (init, sys)

[) \}

\{l determine the size of the network buffer less contingency part -- at least 2 maximum size msgs must be able to fit in the

- to insure that when a $\mathrm{msg}$ is placed in the network buffer

-- there is still room for 1 max size msg.

gen.msg.can.fit $:=($ max.nbuff +1$)-(2 *$ params 10$)$ 


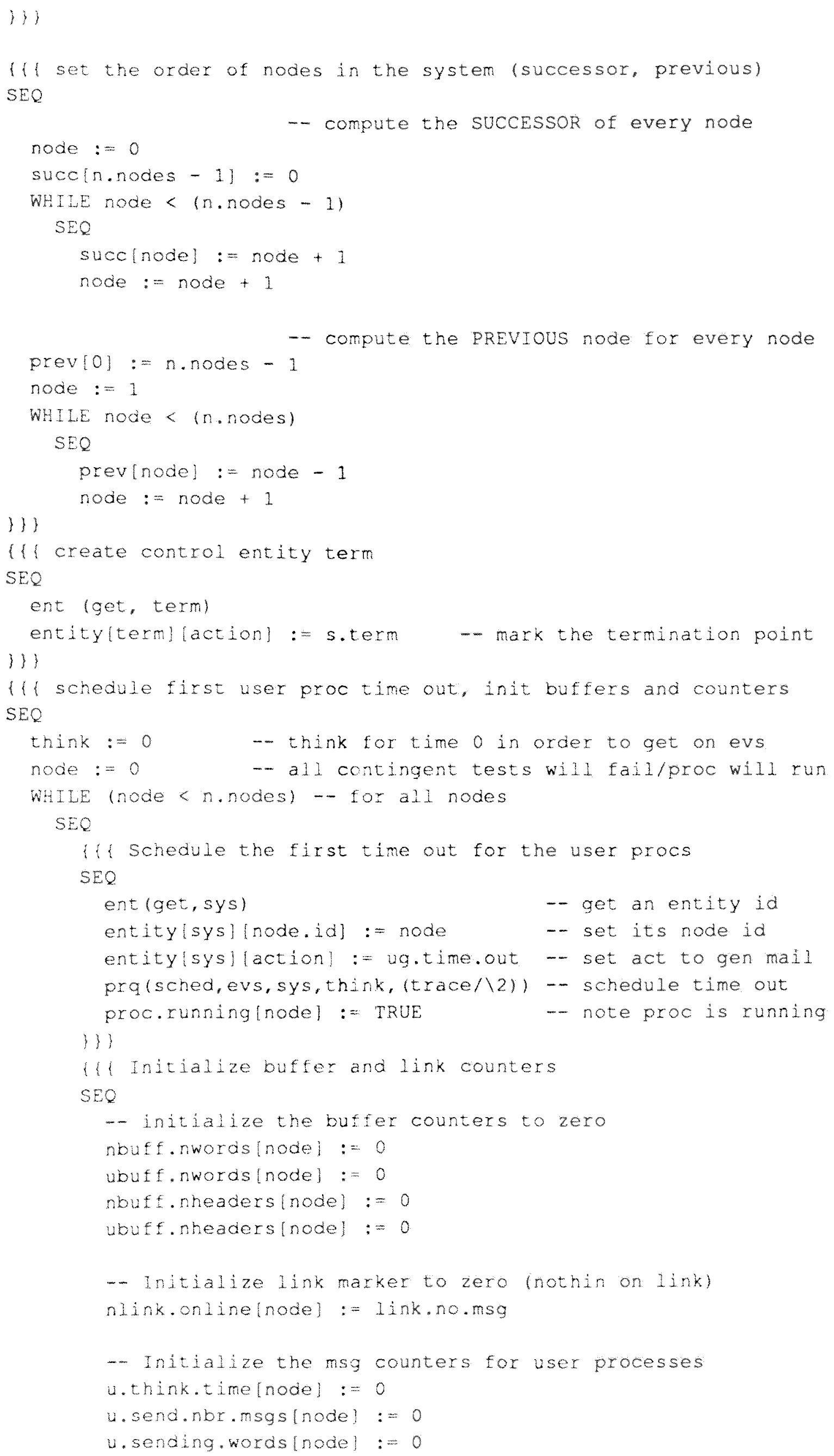




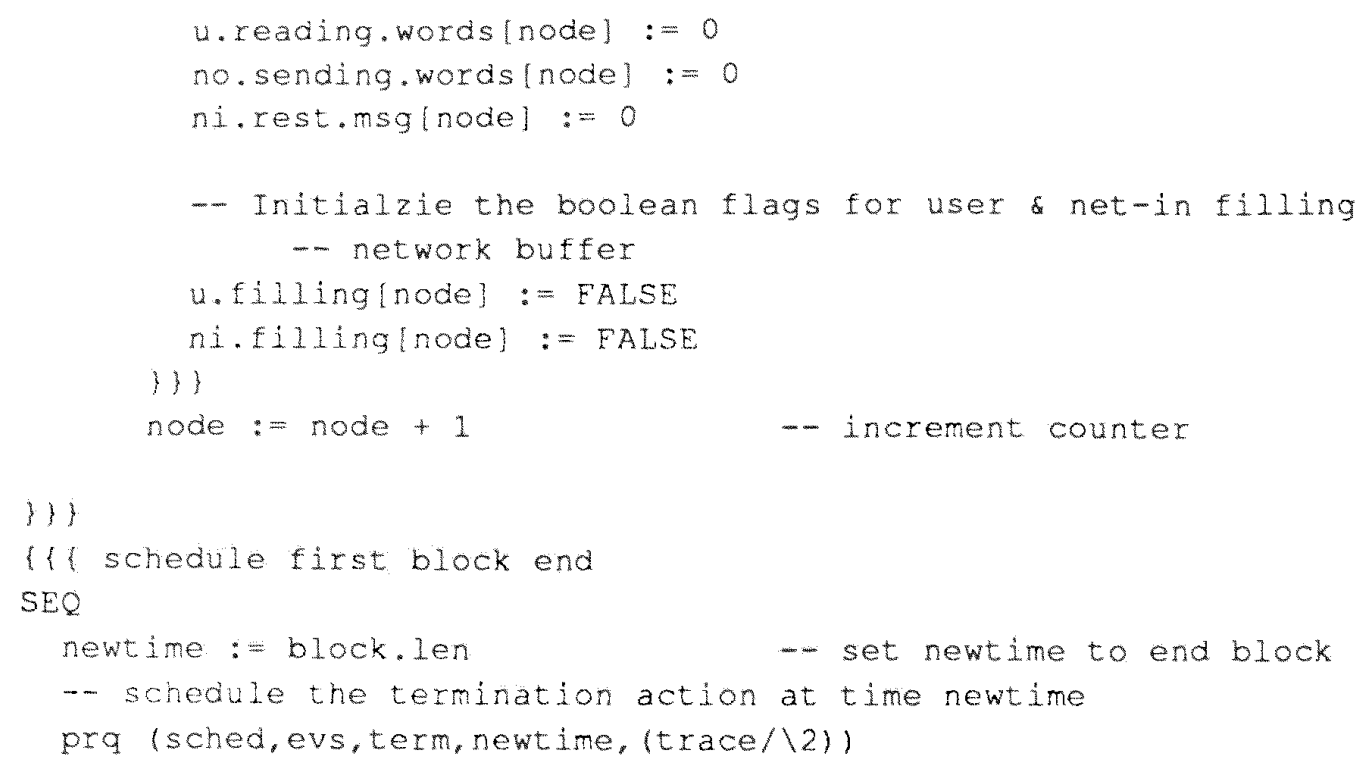


1)

((l Create the user front process (uf)

- get the entity id, assign it to this process, assign

- the node this id, let stats know start state, and

- assign the process to the start state, and place

-- process on Block queue

SEQ

ent (get, sys)

uf [node] := sys

entity[sys][node.id] := node

ens (get, sys, uf.block, prior, (trace/16))

uf.state[node] : = uf.block

prq (sched, blockq[node], sys, clock, (trace/(4))

1)

(I) Create the network receiver (ni)

- get the entity id, assign it to this process, assign

- the node this id. let stats know start state, and

- assign the process to the start state, and place

- process on Block queue

SEQ

ent (get, sys)

nilnodel: : sys

entity[sys] [node.id] := node

ens(get, sys,ni.sleep,prior, (trace/ (16))

ni.state[node] : = ni.sleep

prq (sched, blockq[node], sys, clock, (trace/ 4 ))

) )

(1) create the network transmitter (no)

-- get the entity id, assign it to this process, assign

-- the node this id, let stats know start state, and

- assign the process to the start state, and place

- process on Block queue

SEQ

ent (get.sys)

nolnode] : = sys

entity[sys] [node.id] :: node

ens (get, sys, no. sleep, prior, (trace/ $\backslash 16)$ )

no. state (node) $:=$ no.sleep

pra (sched, blockq[node], sys, clock. (trace/ 4))

1))

node : $=$ node +1

)1)

1)

opus $=5 i m \cdot s i m$ 


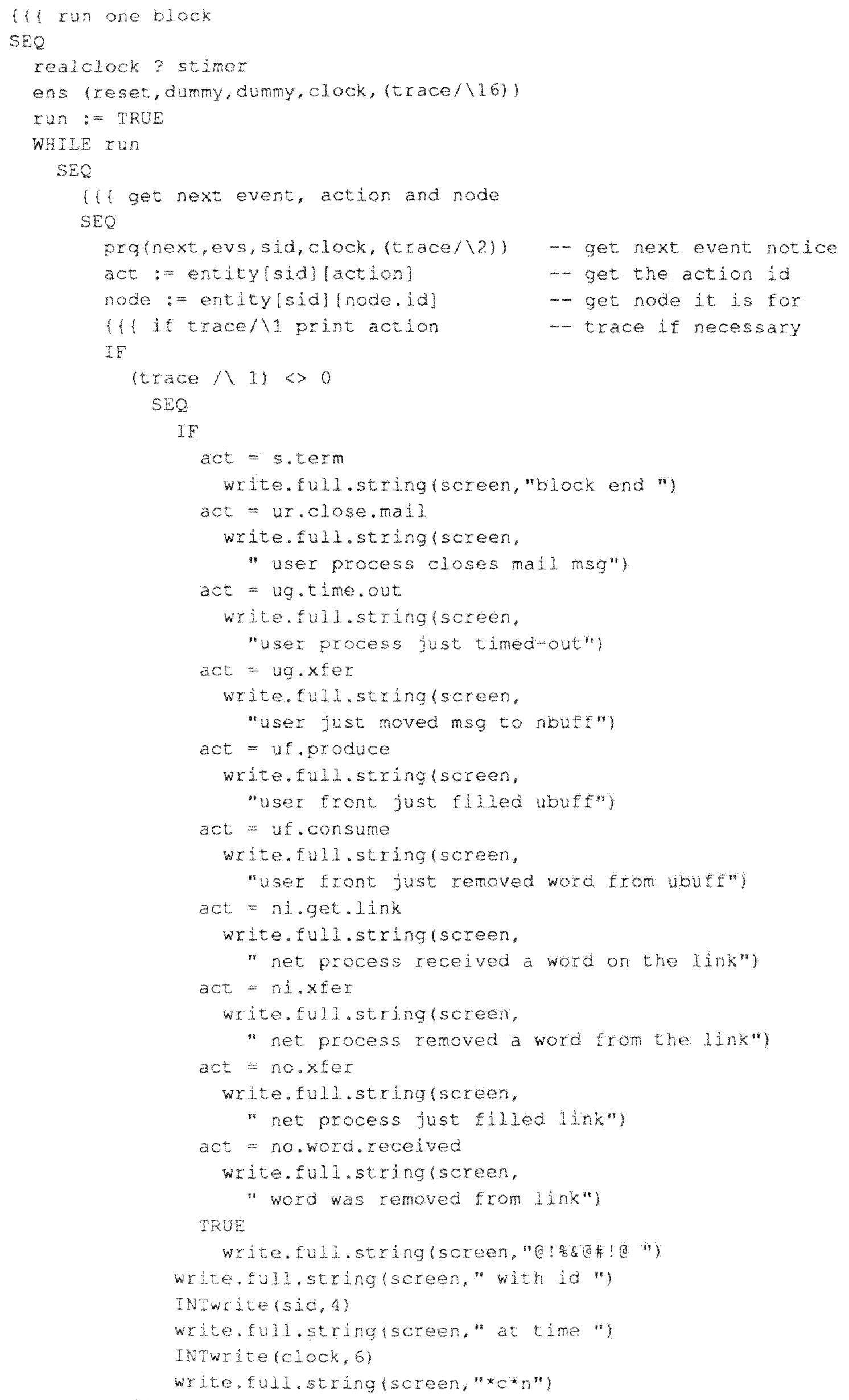
TRUE 


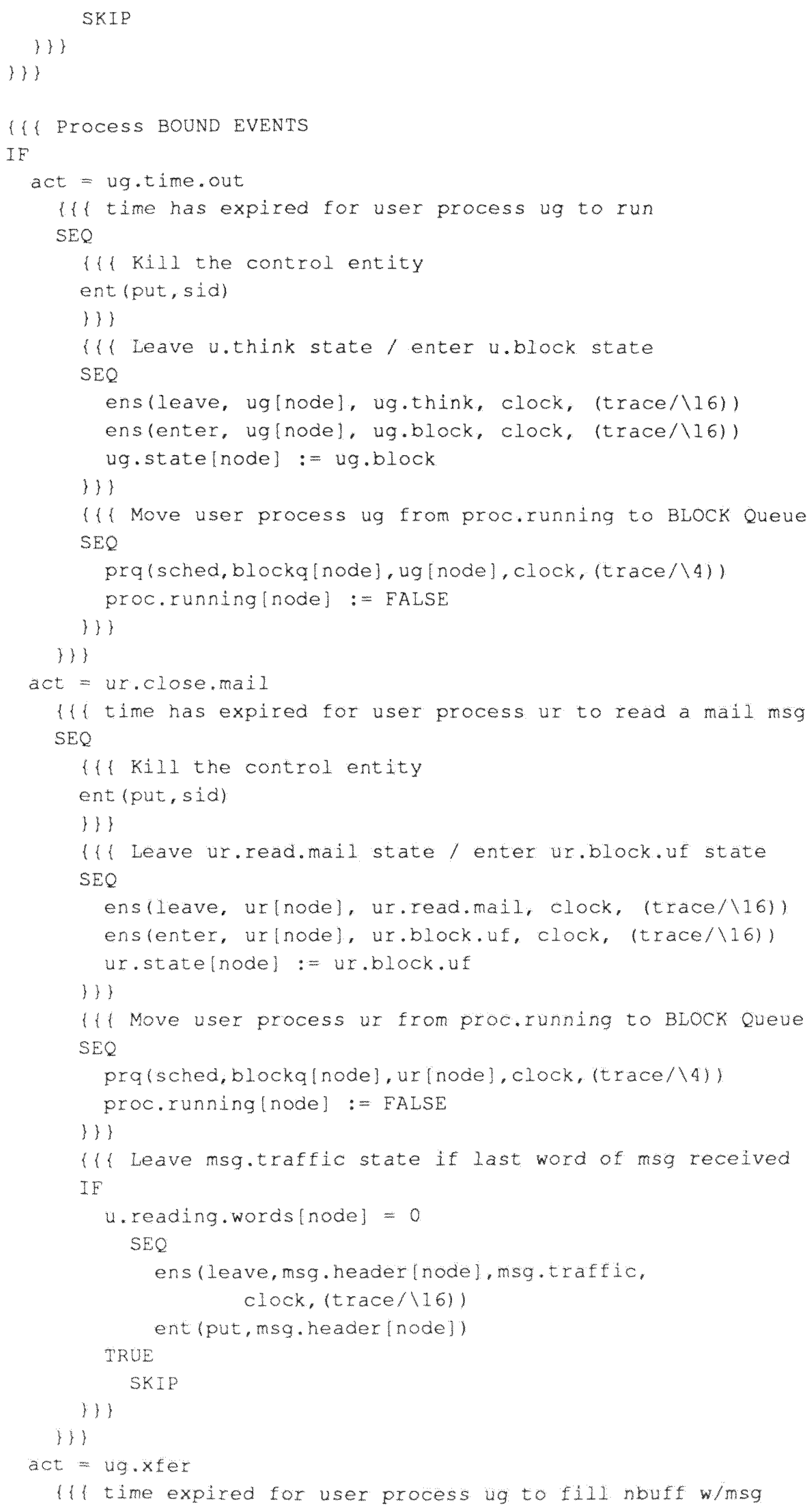




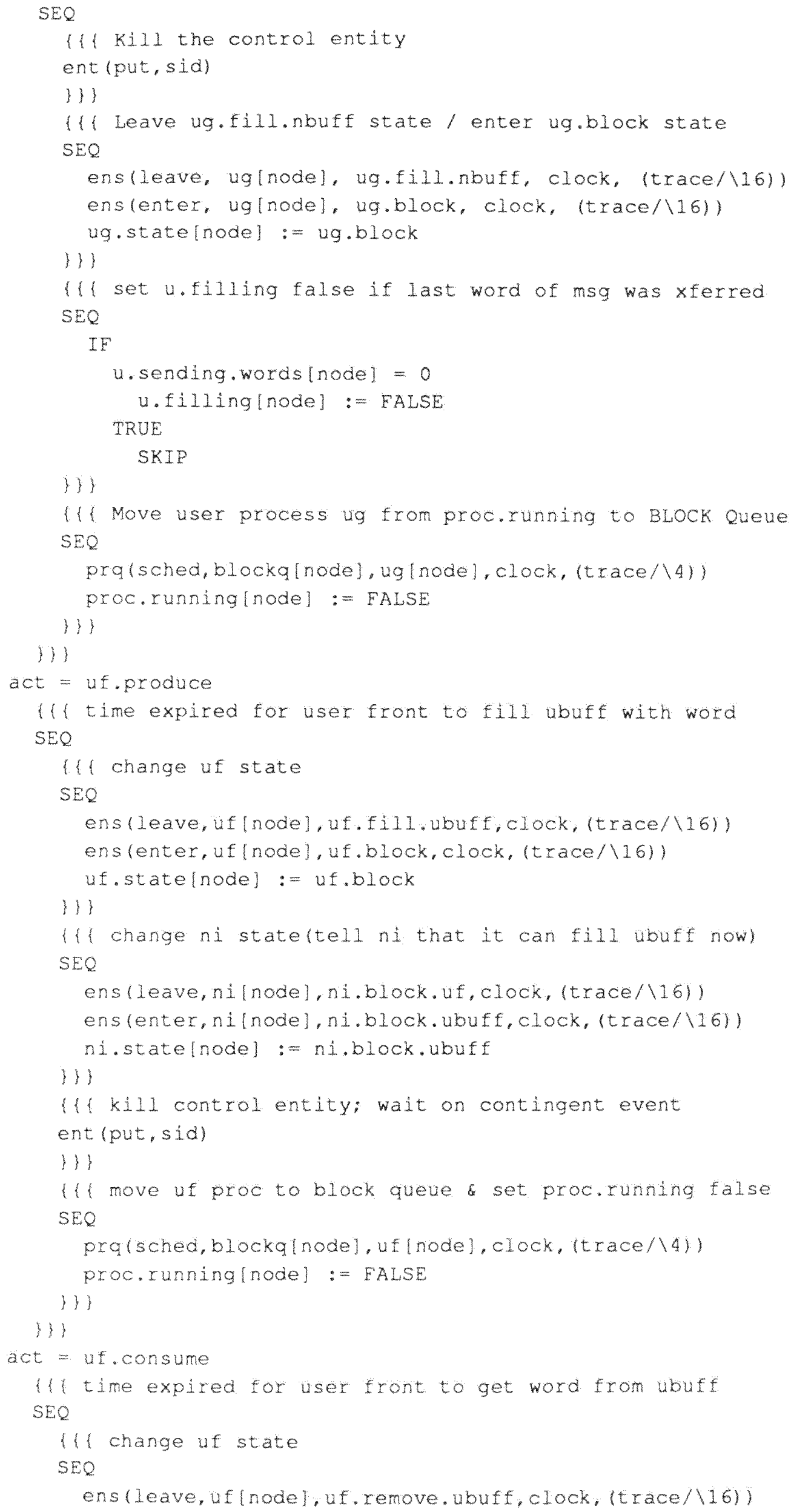




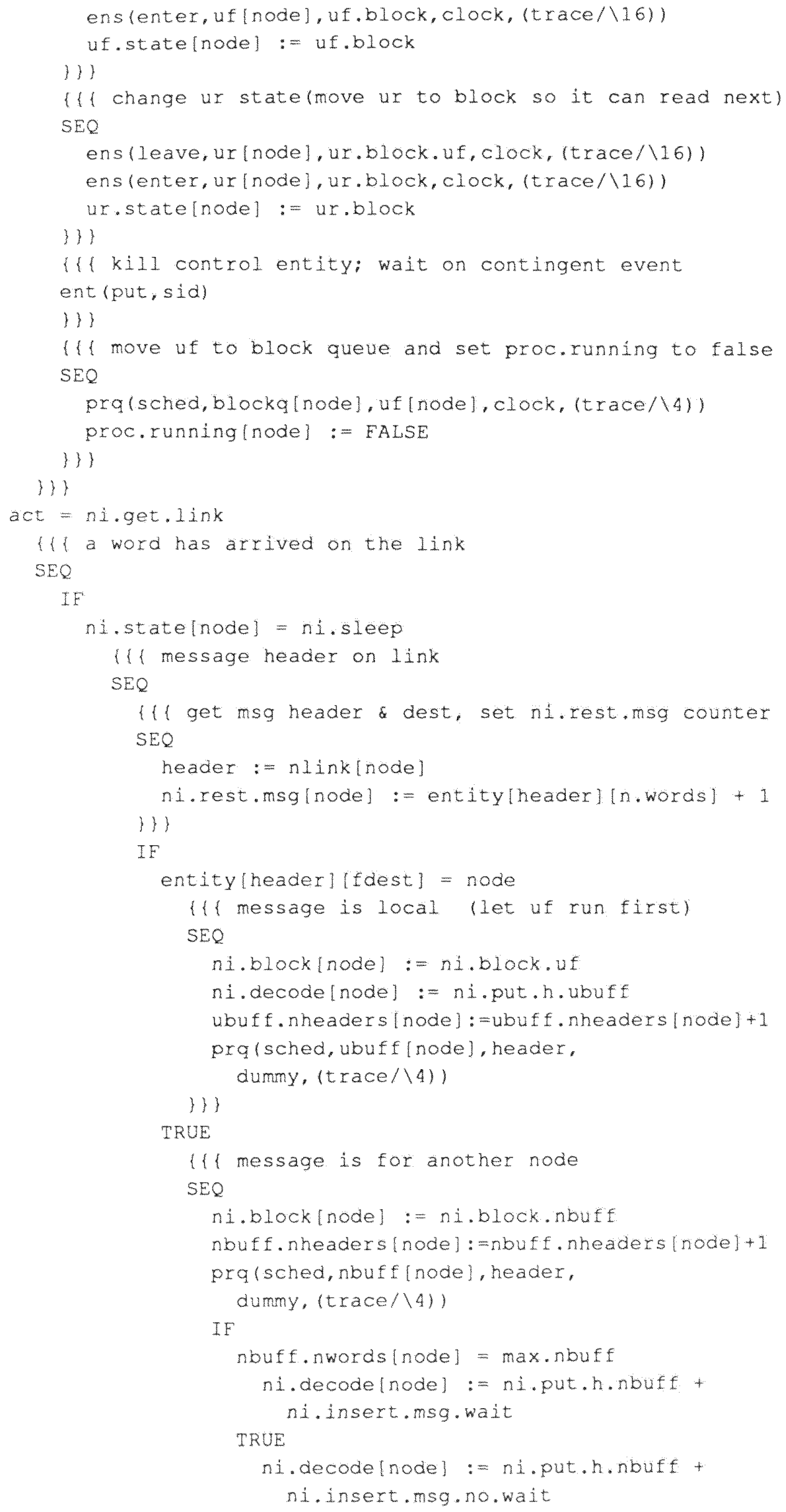


i) \}

$\{1$ enter block state

$S E Q$

ens (leave, nilnode], ni.sleep, clock, (trace/ (16))

ens (enter, nilnode], ni.block [node]. clock, (trace/\16))

ni.state[node] $:=$ ni.block[node]

1)

(fi kill control entity

ent (put, sid)

) )

3) )

TRUE -- ni is waiting on the link

(/) one word of the message is on link

SEQ

$\{1$ enter block state

SEQ

ens (leave,ni[node], ni, wait.on. Iink, clock, (trace/ 161$)$

enslenter,ni(node],ni.block (node). clock, (trace/(16))

ni.state[node] : = ni.block [node]

H)

II set time to decode word to time needed to place - word in nbuff or ubuff

$I F$

ni.block[node] $=$ ni.block. ubuff

ni.decode[node] :=ni.put, w. ubuff

nbuff. nwords [node $]=\max$.nbuff

ni.decode[node] :=ni.put.w.nbuff +

ni.insert.msg. wait

TRUE

ni. decode (node) : =ni.put. w. nbuft + ni.insert.msg.no.wait

3)

\{l kill control entity

ent (put,sid)

1)

3) ?

3)

act $=$ ni.xfer

(1) time expired to move a word from link to buffer

SEQ

IE

ni.rest.msglnode] $>0$

\i message not complete, enter wait on link SEQ

ens (leave, nilnode], ni.state [node], clock, (trace/ $(16))$

enslenter, nilnodel, ni. wait.on.link, clock, (trace/(16))

ni.state[node] $:=n i$.walt.on. Iink

H)

TRUE 


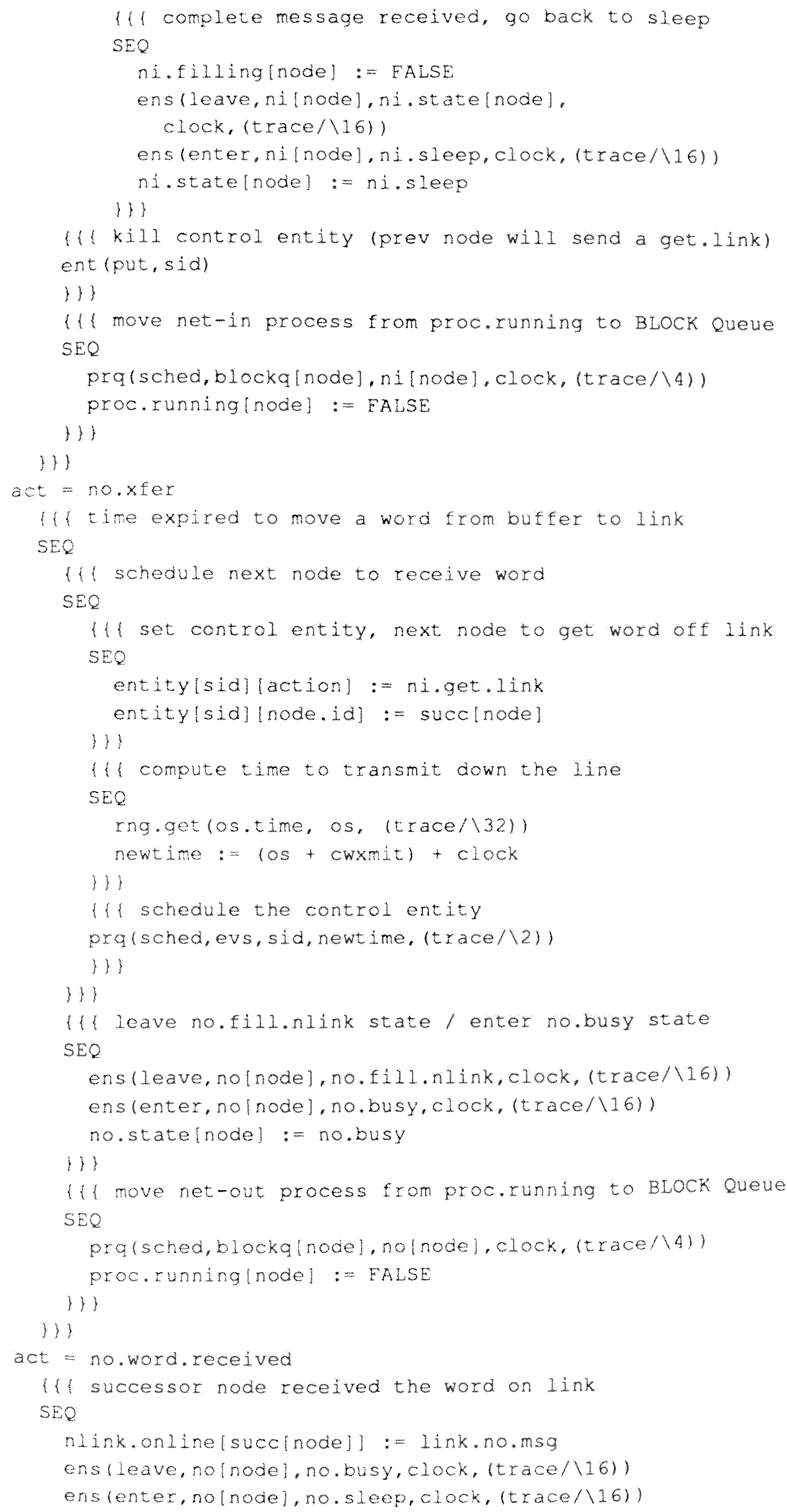




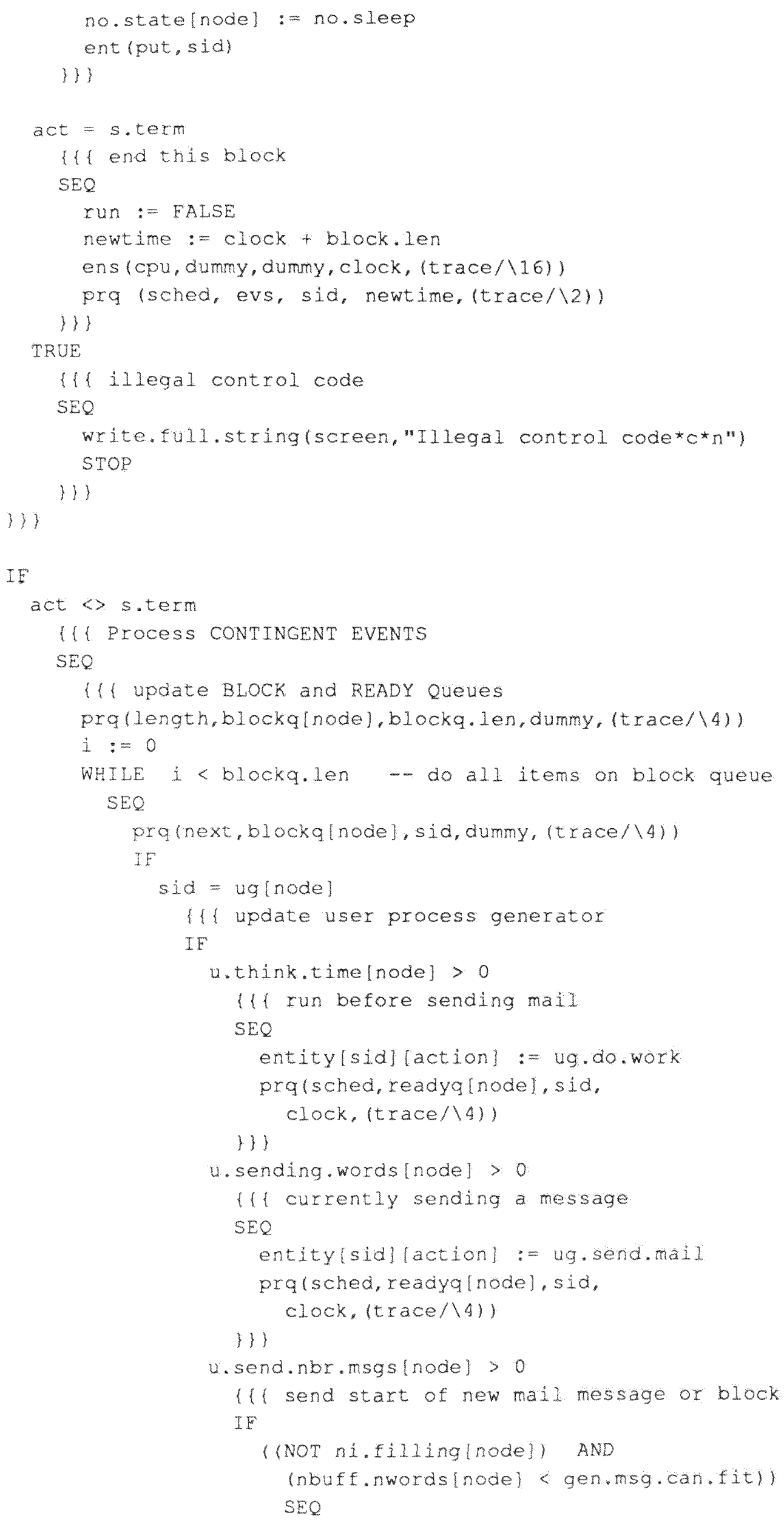


u.filing [node] := TRUE

entity[sid][action] := ug.send.mall

pralsched, readyq[node], sid.

clock, (trace/ $\backslash 4)$ )

TRUE - -msg can't be moved, go on BLOCK queve

prq (sched, blockq[node], sid,

clock, (trace/ $\backslash 4)$ )

1)

TRUE

If run the application program

$\mathrm{SEQ}$

entity[sid] [action] := ug.do.work

prq(sched, readyalnode], sid.

clock, (trace/ $\backslash 4)$ )

rng.get (nbr.msgs, Len, (trace/ $(32)$ )

u. send.nbr.msgs[node] := len

rng.get (proc.time, think, (trace/ 32$)$ )

u.think.time[node] $:=$ think

b)

))

sid $=u x[$ node $]$

[ll update user process receiver

SEQ

IF

ur.statelnodel = ur.block

if move ur to READY queue SEQ

entity[sid][action] :=ur.get.mail

pra (sched, readyalnode), sid. clock, (trace/ 4 ))

) 1)

TRUE

Ifi place ur back on Block queve

pralsched, blockq[nodel, sid, clock, (trace/ $\backslash 4)$ )

1)

1)

sid $=$ uf [node]

i $\{$ update user front process

SEQ

IF

ni.state (node $=n i . b l o c k$. uf $-n$ i has priority SEQ

entity[sid][action] :=uf.put.ubuff

pro (sched, readyqlnode], sid,

clock, (trace/ $\backslash 4)$ )

(ur. state[node] $=$ ur.block. uf) AND

(ubuff.nwords[node] $>0$ )

SEQ

entity[sid][action] := uf.get.ubuff

pra (sched, readyalnodel, sid,

clock, (trace/ $(4))$ 
TRUE

prq(sched, blockglnode], sid, clock, (trace/ $\backslash 4))$

3)

sid $=$ ni[node]

11 update net-in process

IF

((ni.state[node] $=$ ni.block. ubuff) AND (ubuff.nwords[node] < max.ubuff)) AND (NOT u.fillinglnodel))

SEQ

ni.filing[node] := TRUE

entity[sid] [action] :=ni.put.ubuff

prq(sched, readyqlnodel, sid,

clock, (trace $(4)$ )

(1ni.state[node] $=n \perp . b$ lock.nbuff) AND

(nbuff.nwords[node] < max.nbuff)) AND

(NoT u.filling(node]))

SEQ

ni. Eillinglnodel := TRUE

entity[sid][action] := ni.put.nbuff

prq(sched, readyolnodel, sid,

clock, (trace/ $\backslash 4)$ )

TRUE - - net-in can't run,go back on BLock queve

prq (sched, blockqlnodel, sid, clock, (trace/ 4$)$ ) 1)

sid $=$ nolnode]

1\} update net-out process

IF

(\{nlink.online[succ[node] ] = link.no.msg) AND

(nbuft.nwords[node] $>0)$ )

SEQ

entity[sid] [action] : = no.send. word

prqisched, readyqlnode], sid.

clock, (trace/ $\backslash 4)$ )

TRUE

prq(sched, blockq[node], sid, clock, (trace/44)) 1)

TRUE

write.full.string(screen,

"Illegal control entity on BLOCK queve*c*n")

$1:=1+1$

)1)

$1($ set one process running if necessary/possible

prq(length, readyq[node], len, dummy, (trace/ $\backslash 4)$ )

IF

(Ien > 0) AND (proc.running $[$ node $)=$ FALSE)

SEQ

I 1 get next action and proc, set proc.running TRUE SEQ

prq(next, readyq(node), sid, len, (trace/14))

act $:=$ entity[sid]laction]

proc.running(node) := TRUE 


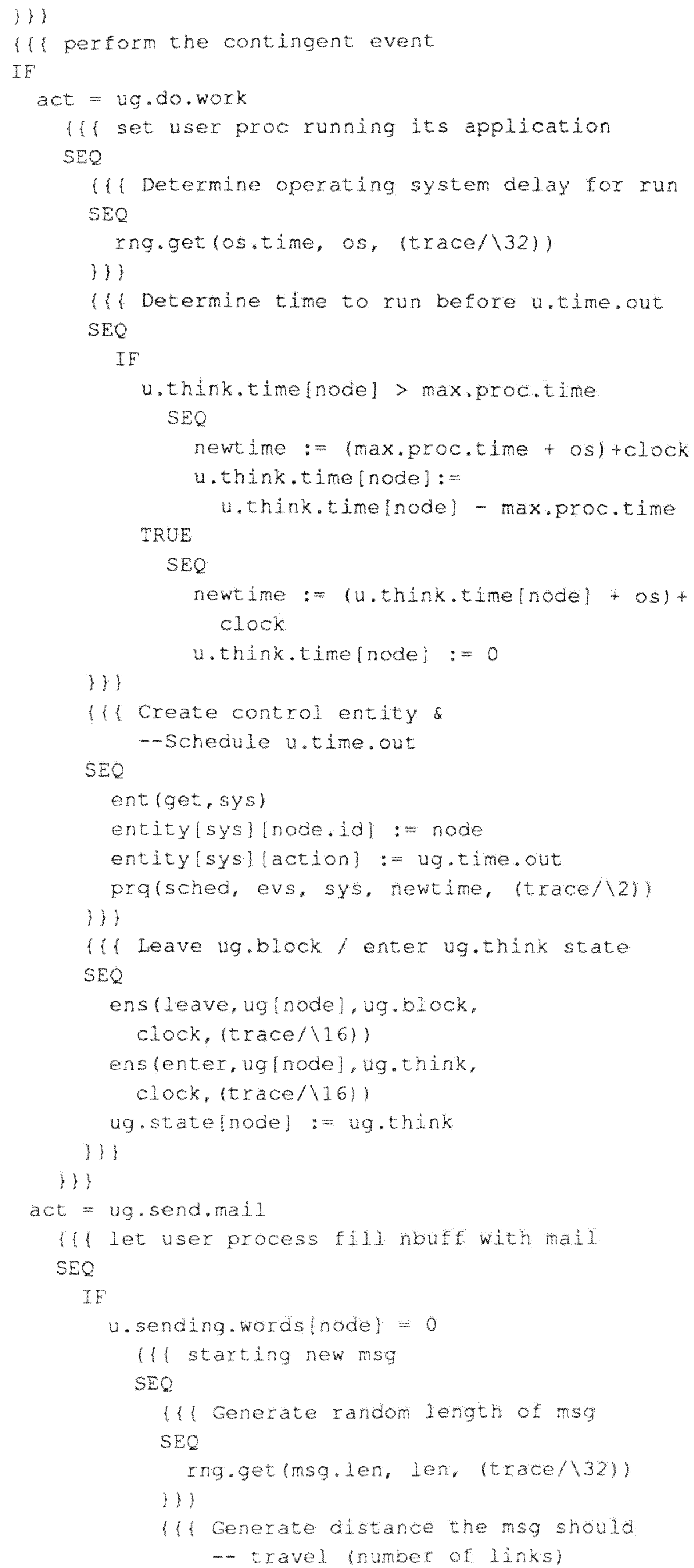




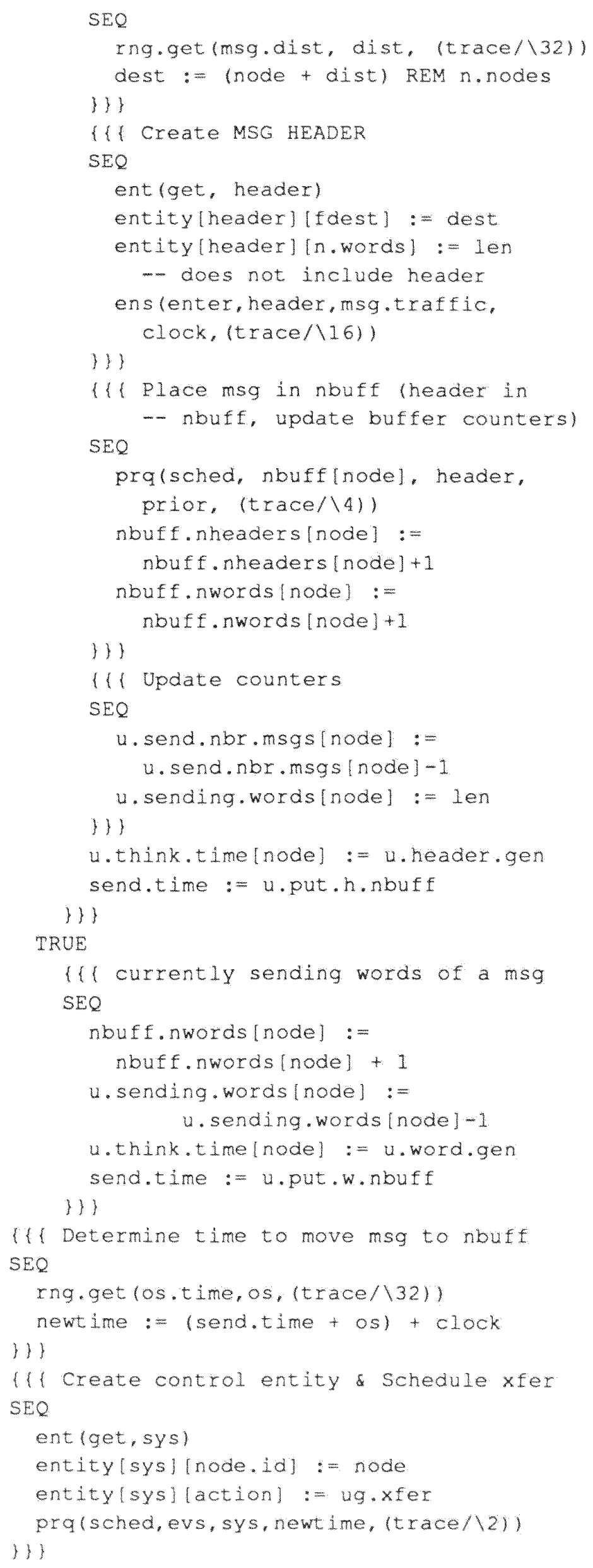


(1/ Leave ug.block / Enter ug.fi11.nbuff SEQ

ens (leave, uglnode], ug,block. clock, (trace/(16))

ens (enter, uglnode], ug.fil1.nbuff, clock, (trace/ \16))

ug. statelnodel := ug. fill, nouff

1)!

1)\}

act $=$ ur.get.mail

(1/ let user process read mall msg waiting SEQ

IF

u.reading.words [node $=0$

(1) get next header from ubuff, set

- counters, set read time

SEQ

prq(next, ubuff [node], header, dumny, (trace/ $\backslash 4)$ )

$u$. reading. words $[$ node $]:=$ entity [header] [n.words]

ubuff.nwords [node] : = ubuff.nwords (node) - 1

ubuff, nheaders [node]: = ubuff, nheaders (node) - 1

msg.header [node] := header

- ent (put, header)

read.time : = u.read.header

)!

TRUE

If update counters, set read time SEQ

ubuff. nwords (node) := ubuff.nwords [node] - 1

u. reading. words [node]:= u.reading.words [nodel-1

read.time := u.read.word

b)

if compute time to read the $\mathrm{msg}$ SEQ

rng.get (os.time.os, (trace/ $\backslash 32)$ )

newtime $:=($ read.time + os) + clock

1)\}

(1) create control entity \& schedule transfer SEQ

ent (get, sys)

entity[sys] [node.id] $:=$ node

entity[sys][action] $:=$ ur.close.mall

prq(sched, evs, sys, newtime, (trace/ $/ 2)$ ) 1)

(l) leave ur.block / enter ur.read.mali state SEQ

ens(leave, ur [node], ur.block,

clock, (trace/(16)) 


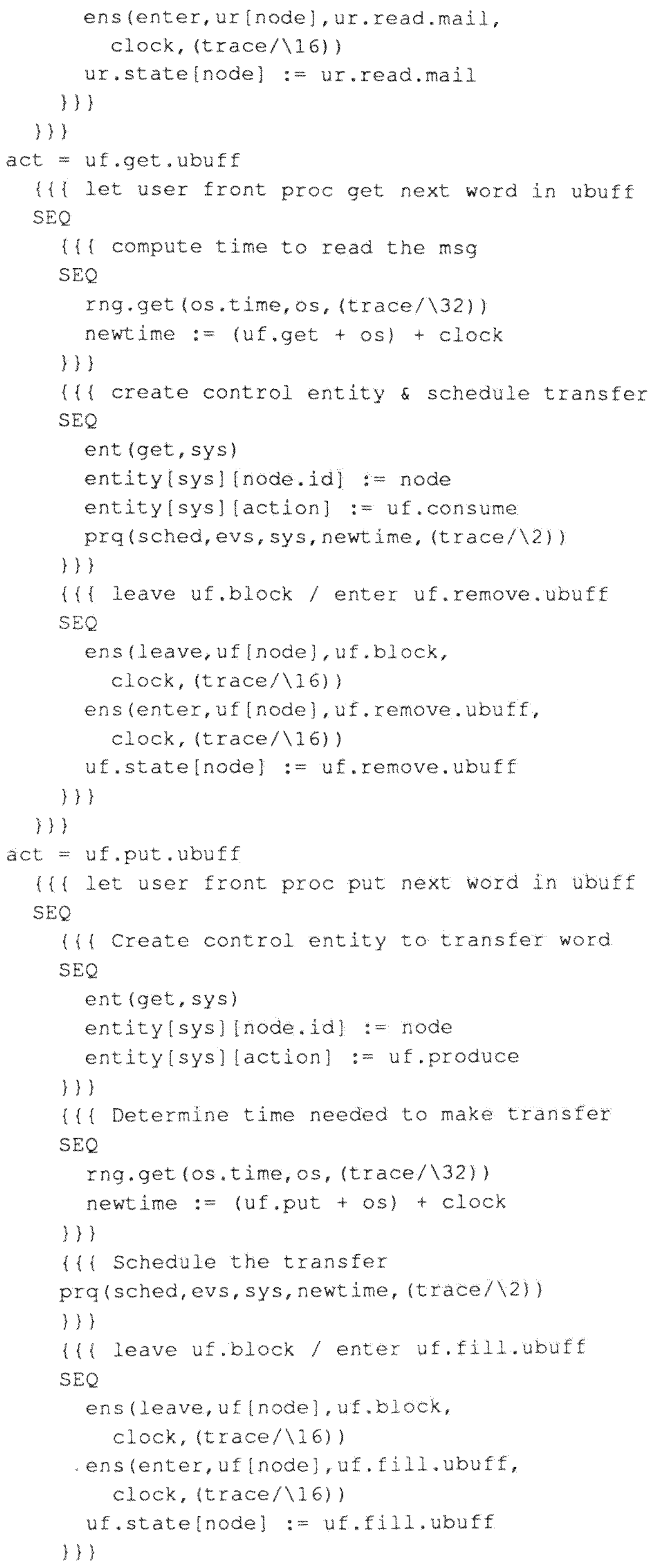


3)

act $=$ ni.put.nbuff

\{ ( l let net-in proc fill nbuff $w /$ word on link SEQ

(l) Create control entity to transfer word SEQ

ent (get, sys)

entity [sys] [node.id] := node

entity[sys][action] :=ni.xfer

(3)

I( Determine time needed to make transfer SEQ

rng.get los.time, os, (trace/ (32))

newtime $:=$ (ni.decode[node] +os) + clock

1)

(f) Schedule the transfer

prq (sched, evs, sys, newtime, (trace/ 2 ))

1) \}

II update word counters

SEQ

nouff.nwords[node] : = nbuft.nwords[node]+1

ni.rest.msg[node] := ni.rest.msg[node] - 1

1)

\{if leave ni.block.nbuff/ enter ni.fill.nbuff SEO

ens (leave, ni [node], ni.block. nbuft, clock, (trace/ \16))

ens(enter, nilnode],ni.fili.nbuff, clock, (trace/ 16))

ni.statelnode] : = ni.fi11.nbuff

1) \}

(1) schedule control entity for previous node SEQ

ent (get,sys)

entity[sys][node.id] := prev[node]

entity[sys][action] := no.word.recelved

prq(sched,evs, sys, clock. (trace/ $\backslash 2)$ )

3)

i)

act $=$ ni.put.ubuff

(1) let net-in proc fill ubuff w/word fron link $\mathrm{SEQ}$

( $/$ Determine time needed to make transfer SEQ

rng.get los.time, os, (trace/ $\backslash 32)$ )

newtime $:=n i$. decode $[$ node $]+\left(\right.$ clock $+\left(3^{\star}\right.$ os $\left.)\right\}$ ) 1)

It Schedule the transfer

SEQ

ent (get, sys)

entity[sys] [node.id] := node

entity[sys][action $]:=n i \cdot x t e r$

prq(sched, evs, sys, newtime, (trace/12))

1) 


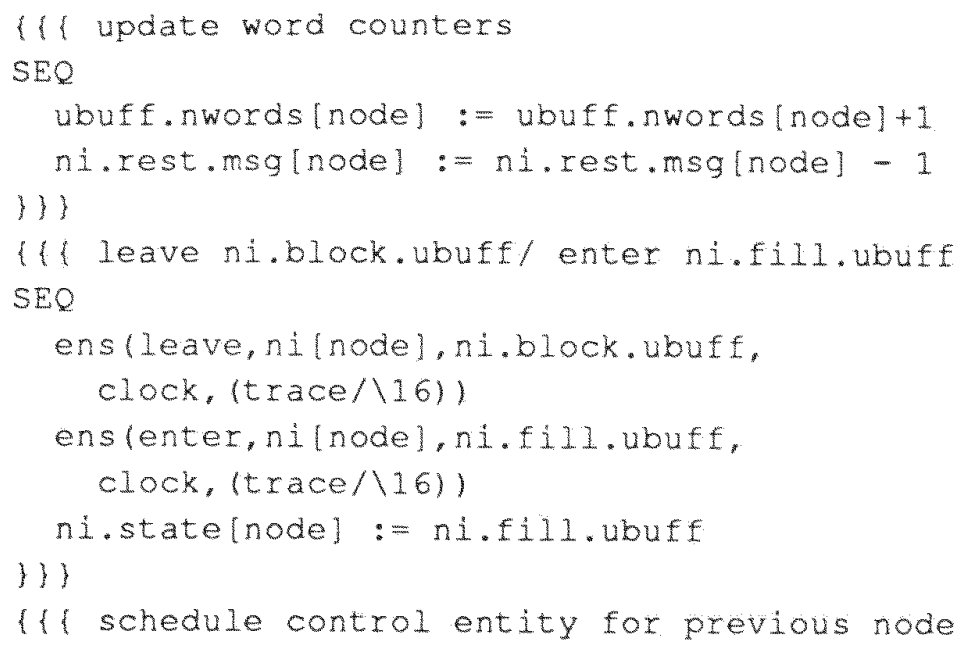

Ifl schedule control entity for previous node SEQ

ent (get, sys)

entity[sys] [node.id] := prev[node]

entity[sys][action] := no.word.received

prq(sched, evs, sys, clock, (trace/ 2 ))

H)

1) )

act $=$ no. send. word

\{ ( let net-out process place word on link SEQ

IE

(no. sending, words [node] > 0 )

- still sending a msg

(if put word on link (decrement counters) SEQ

no. sending, words [node]:= no. sending. words [node]-1

nbuff.nwords [node] :=

nbuft.nworas [node] - 1

nlink. on Iine $[$ succ $(n o d e)]:=$ link. word.msg

Y)

TRUE

- - send start of $\mathrm{msg}$

$1 / 1$ put header on link, decrement counters SEQ

(if move the header from nouff to

-- nilink, update sending.words

SEQ

prqinext, nbuff [node], header, prior, (trace/ 4 ))

no. sending. words (node) :=

entity [header] [n. words]

nlink [succ[node]] := header

) H

$\{1\}$ update counters controling buffers - and links

SEQ

nbuff, nheaders[node] : =

nbuff. nheaders [node] - 1 


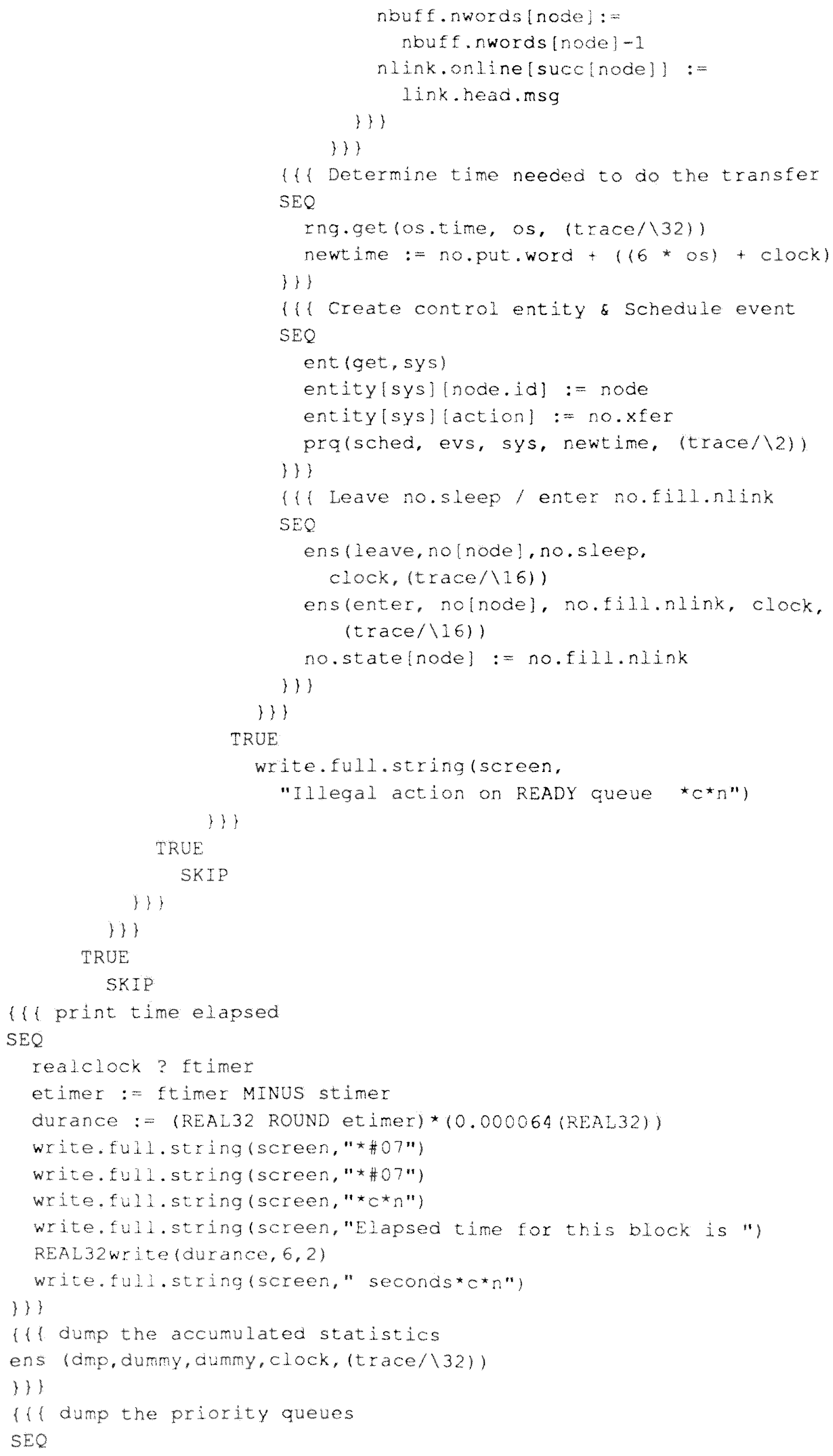




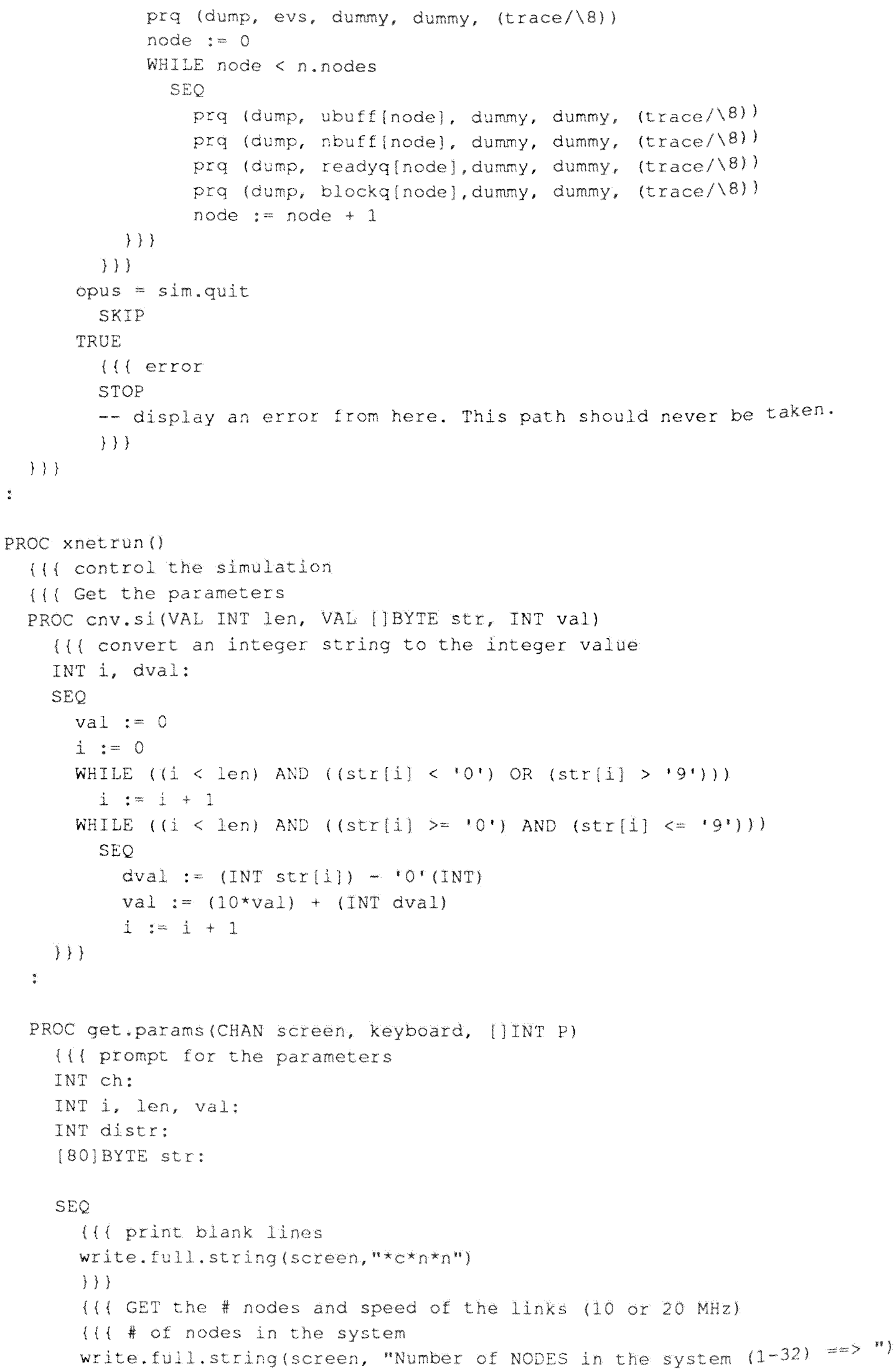




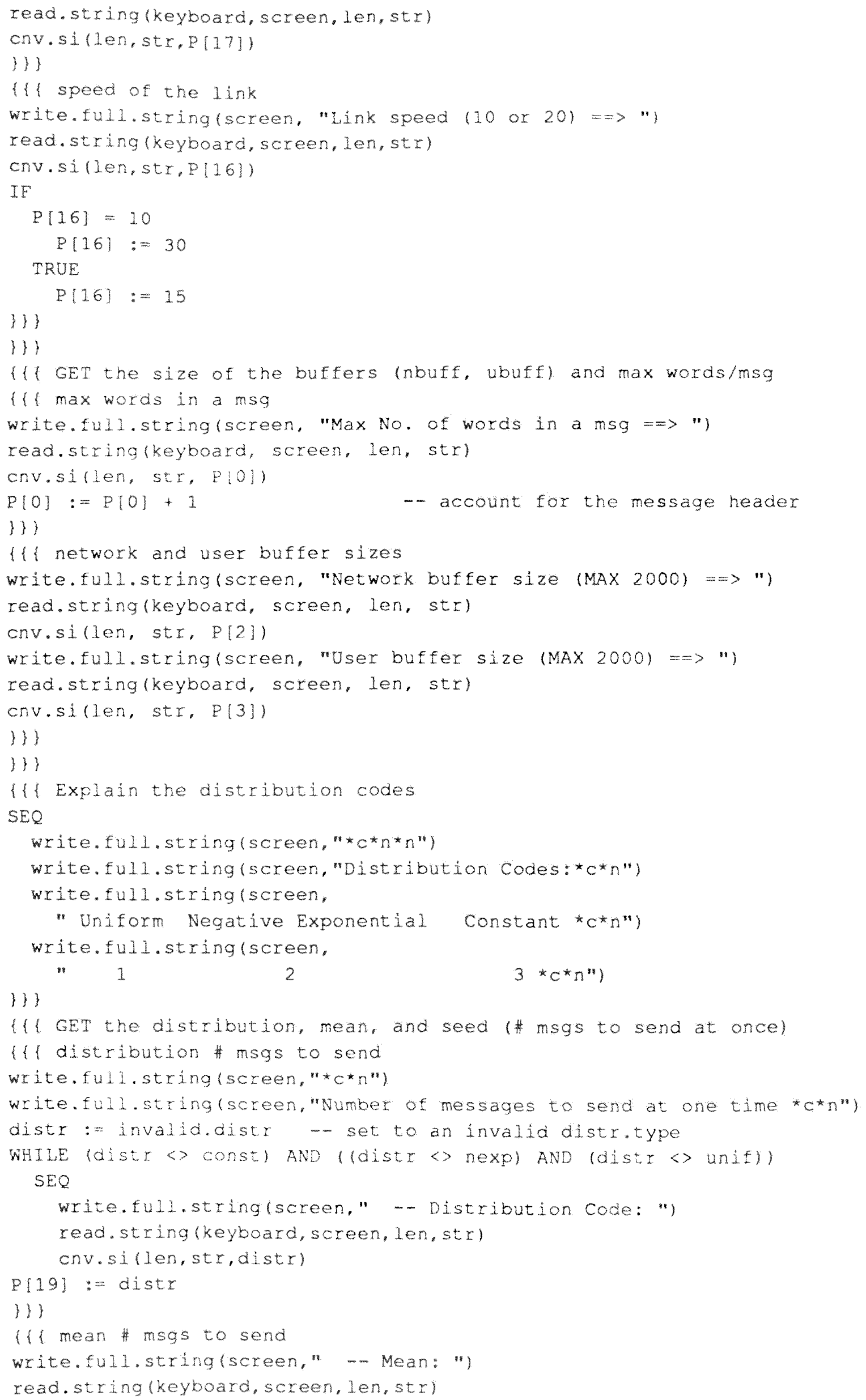




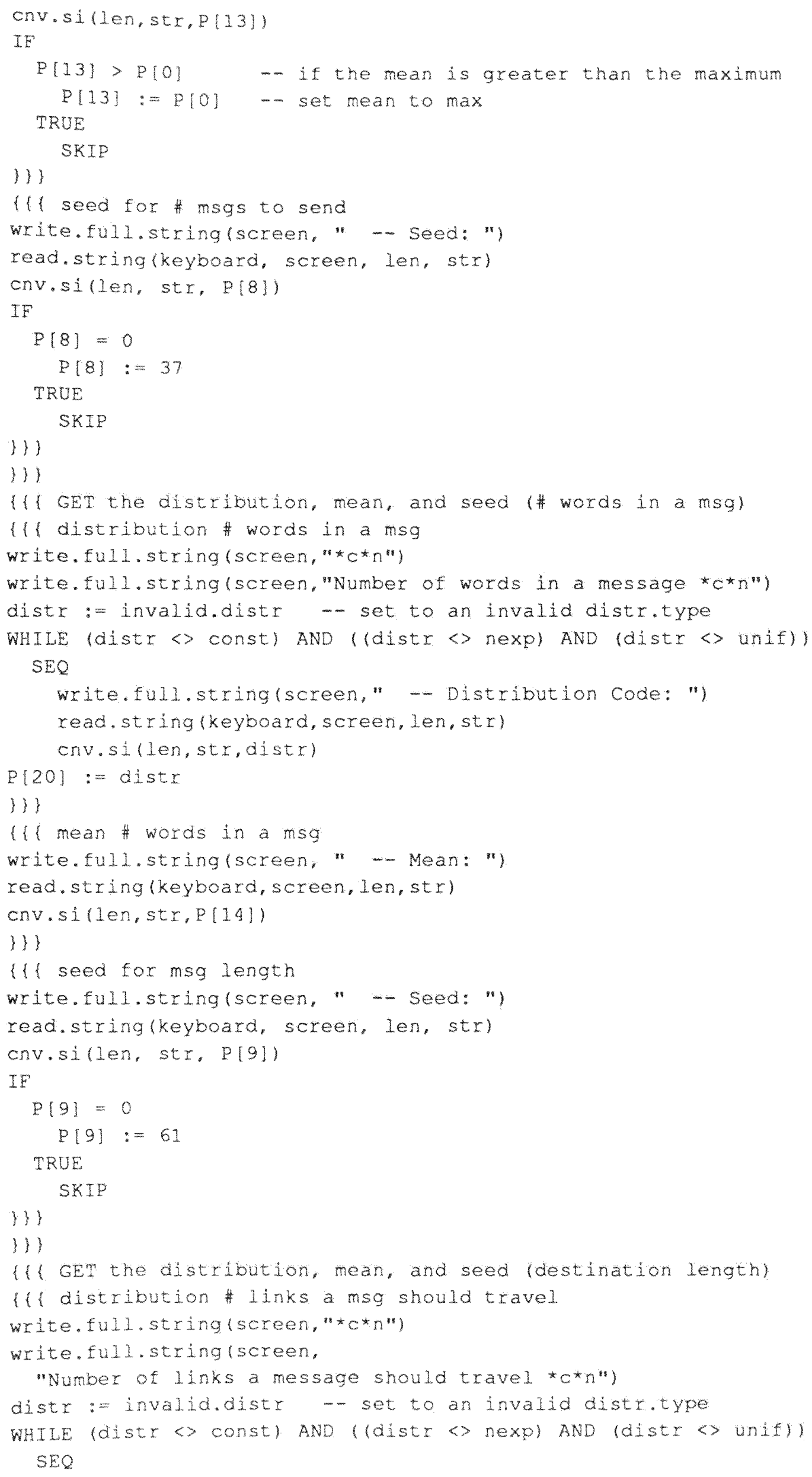




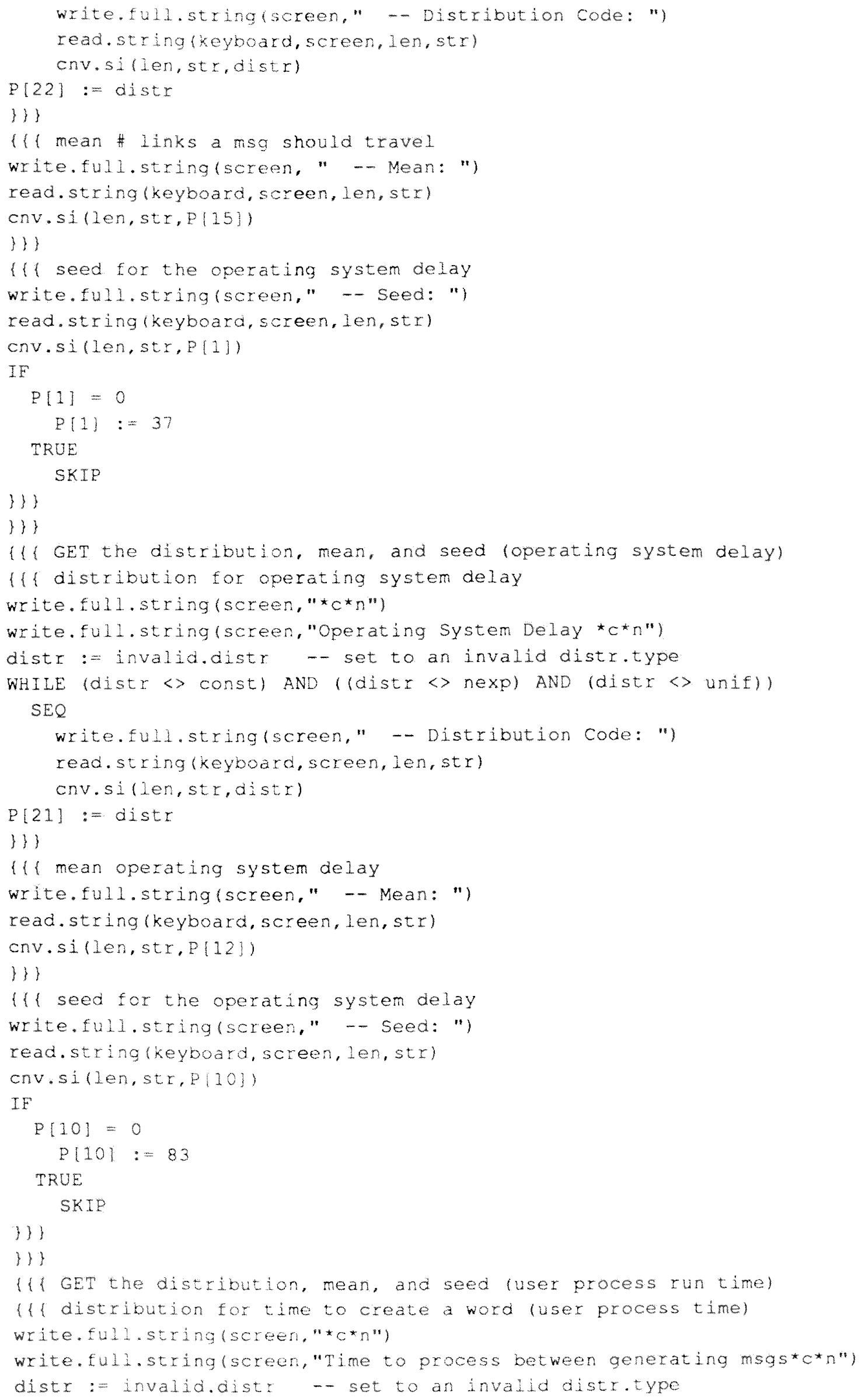




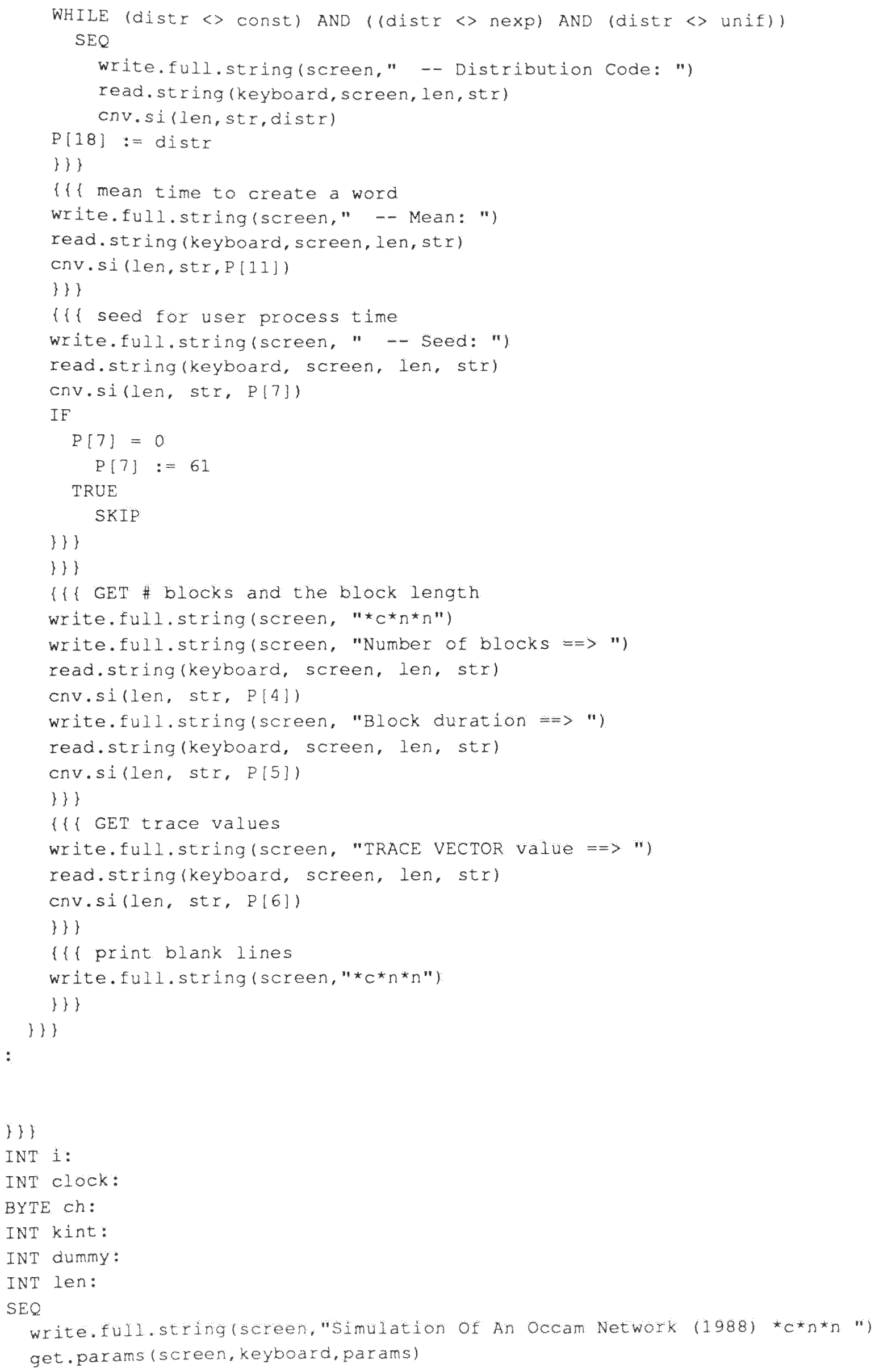


(1) initialize the priority queue objects

-- the priority queve objects are self initializing

INT $c, 01, c 2,03$, node:

SEQ

node $:=0$

$c:=1$

c1: : max.nodes * 1

c2 : = max.nodes * 2

c3: $=\max$.nodes *3

- ASSIGN THE RRIORITY QUEUES

WHILE node $<\max$. nodes SEQ

ubuff[node] := c - - start at 1 (note: evs is queve o)

nbuff[node] : = c + cl - - get next queve number for this node

readyq[node] : $=c+c 2$ - get next queve number for this node

blockq[node] : =c $+c 3 \quad-$ get next queve number for this node

node : $=$ node +1

-- get next node number

$c:=c+1$

-- increment by one

) 1)

(l) initialize the RNG objects

$S E Q$

rng. init (nbr.msgs, distr.gen.msgs, params [13], params (8])

rng.init (proc.time, distr.proc.time,params [11], params [7])

rng.init (msg.len, distr.msg. len, params [14], params [9])

rng.init (os.time, oistr.ostime, params (12), params (10))

rng.init (msg.dist, distr.msg.dist,params [15], params [1])

1)\}

xnetsim(sim.init, clock)

kint $:=1$

WHILE (kint $<=$ n.blocks)

$\{\{$ Run simulation for another block

SEQ

xnetsim(sim.sim, clock)

write.full.string (screen, "** $\mathrm{C}^{*} n$ )

write.full.string (screen, "BLock \#")

INTwrite (kint, 3)

write, fuli.string(screen, "* $\left.c^{*} n "\right)$

kint $:=k i n t+1$

1) 3

xnetsim(sim.quit, clock)

(1) terminate the statistics process

SEQ

ens (quit, dummy, dummy, clock, (trace/16))

\}) \}

(1) terminate the priority queve objects

SER

pra(quit, evs, dummy, dumy, (trace/(2))

$i:=1$

WHILE $1<\max$ sys.queues 


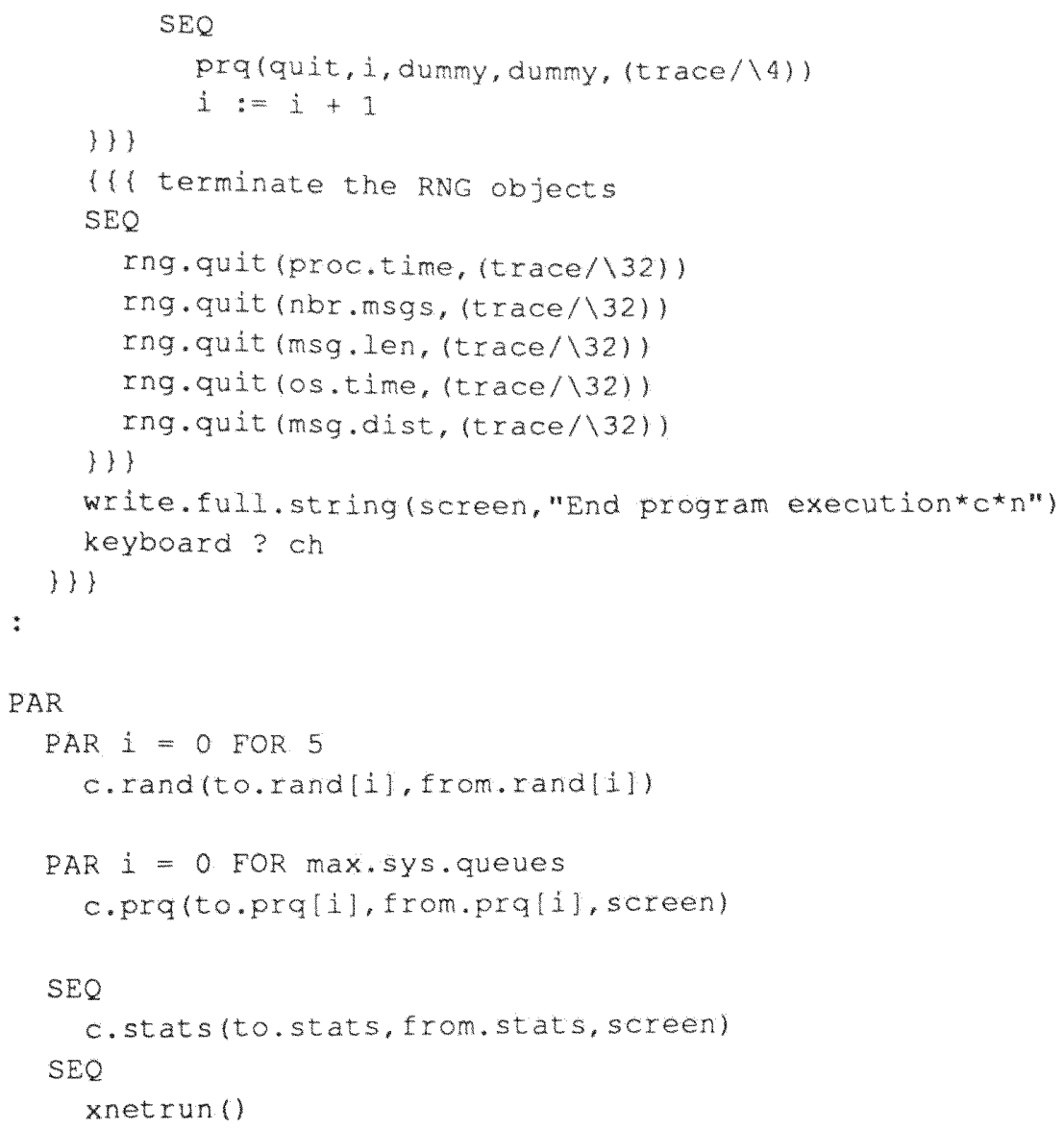




\section{LIST OF REFERENCES}

1. Banks, Jerry, and Carson, John S. Discrete-Event System Simulation. Englewood Cliffs: Prentice-Hall, 1984.

2. Comfort, J.C. and Raja Gopal, R., "Environment Partitioned Distributed Simulation With Transputers". Proceedings of the 1988 Winter simulation conference, pp. 103-108, Feb. 1988.

3. Garcia, Albert B., Shaw, Wade H., "Transient Analysis of A Store-And-Forward Computer-Communications Network" proceedings of the 1986 Winter Simulation conference. pp. 752-760, Washington, D.C., Dec. 1986.

4. INMOS Transputer Reference Manual. INMOS Ltd. 72-TRN 006-03, Bristol, UK, 1987

5. MacDougall, M.H. Simulating Computer System Techniques and Tools. Cambridge: The MIT Press, 1987.

6. Shannon, R. E. System simulation: The Art and Science. Englewood Cliffs: Prentice-Hall, 1975.

7. Silberschatz, Abraham, and Peterson, James L. operating System concepts. New York: Addison Wesley Publishing Company, 1988 .

8. Stuck, Bart W. "Calculating the Maximum Mean Data Rate in Local Area Networks". Computer, May 1983, pp. 72-76.

9. Qiang, Li, William B. Feild Jr., and Donald Klein. "Implementation of a Transputer Ring Network and a Deadlock Prevention Algorithm". Proceedings from the 3rd U.S. Occam User Group Meeting, Sept. 1987.

10. Qiang, Li, William B. Feild Jr., and Donald Klein. "Channel Design Primitives in Occam". Broceedings from the 3rd U.S. Occam User Group Meeting, Sept. 1987. 


\section{VITA}

Master's Thesis Title:
A SIMULATION OE A MESSAGE PASSING PROTOCOI FOR A NETWORK OE TRANSPUTERS

Janice R. Glowacki

Born in Miami, Florida, on January 11, 1963

Attended Sable Palm Elementary, John F. Kennedy Junior High, Highland Oaks Junior High, and North Miami Beach Senior High, all in Miami, Florida.

Attended Diablo Valley College in Pleasant Hill, California, from September 1980 to December 1981.

Attended Florida International University in Miami, Florida, from August 1982 to December 1986 at which time a Bachelor of Science degree was awarded. Majored in Computer Science.

Attended Florida International University in Miami, Florida, from January 1987 to date for graduate studies.

Member of Association of Computing Machinery.

Author of "A Simulation of a Store-and-Forward Distributed Network of Transputers" for the Proceedings of the 1988 Winter Simulation Conference (San Diego, California).

School of Computer Science

September 16, 1988

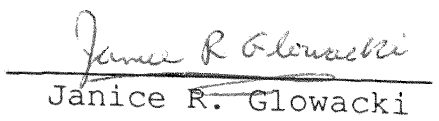

